Supporting Information for:

\title{
Assembling Pentatopic Terpyridine Ligands with Three Types of \\ Coordination Moieties into a Giant Supramolecular Hexagonal \\ Prism: Synthesis, Self-Assembly, Characterization, and \\ Antimicrobial Study
}

Heng Wang, ${ }^{\dagger, \#}$ Chung-Hao Liu, ${ }^{\ddagger, \#}$ Kun Wang,, ,ø Minghui Wang, ${ }^{\dagger}$ Hao Yu, " Sneha Kandapal, ${ }^{\S}$ Robert Brzozowski,, Bingqian Xu, ${ }^{\S}$ Ming Wang, " Shuai Lu, ${ }^{\dagger}, \perp$ Xin-Qi Hao, ${ }^{\perp}$ Prahathees Eswara, ${ }^{\nabla}$ Mu-Ping Nieh, ${ }^{*},+$ Jianfeng Cai, ${ }^{*}, \dagger$ Xiaopeng $\mathrm{Li}^{*}, \dagger$

${ }^{\dagger}$ Department of Chemistry, University of South Florida, Tampa, Florida 33620, United States

*Polymer Program, Institute Materials Science, Department of Chemical \& Biomolecular Engineering,

University of Connecticut, Storrs, Connecticut 06269, United States

IIDepartment of Mechanical Engineering, University of Michigan, Ann Arbor, Michigan 48109, United States

${ }^{\S}$ Single Molecule Study Laboratory, College of Engineering and Nanoscale Science and Engineering Center,

University of Georgia, Athens, Georgia 30602, United States

"State Key Laboratory of Supramolecular Structure and Materials, College of Chemistry, Jilin University,

Changchun, Jilin 130012, China

$\nabla$ Department of Cell Biology, Microbiology and Molecular Biology, University of South Florida, Tampa, Florida 33620, United States

${ }^{\perp}$ College of Chemistry and Molecular Engineering, Zhengzhou University, Zhengzhou, Henan 450001, China 


\section{Table of Contents}

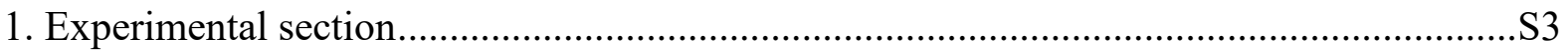

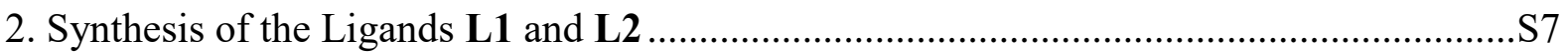

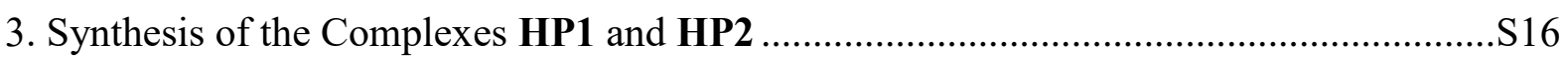

4. ${ }^{1} \mathrm{H}$ NMR, DEPTQ ${ }^{13} \mathrm{C}$ NMR, 2D COSY NMR, 2D NOESY NMR, and ESI-MS spectra of

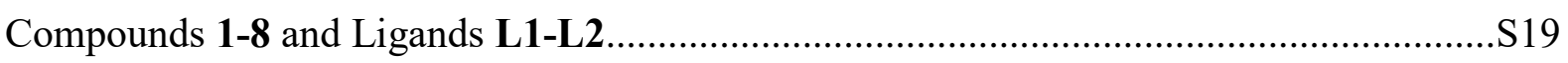

5. ${ }^{1} \mathrm{H}$ NMR, DEPTQ ${ }^{13} \mathrm{C}$ NMR, 2D COSY NMR, 2D NOESY NMR, 2D DOSY NMR, ESI-

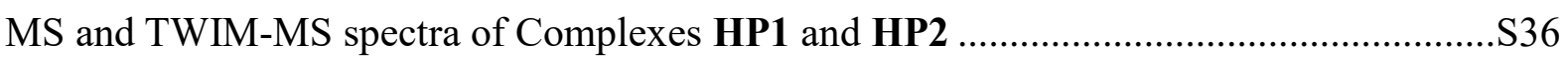

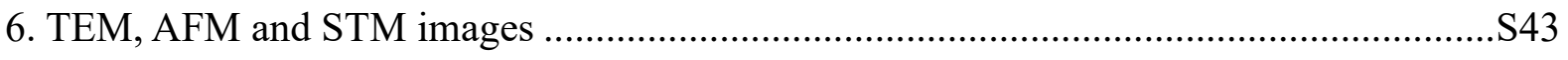

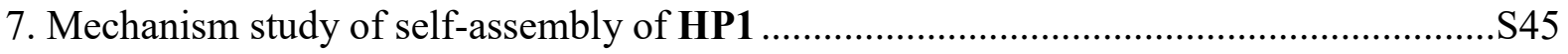

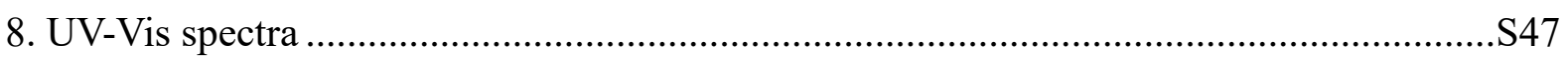

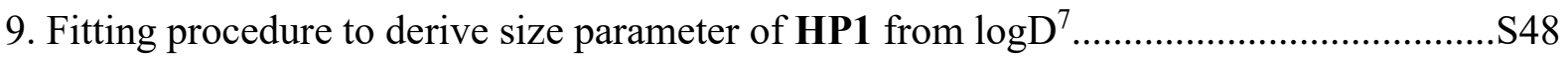

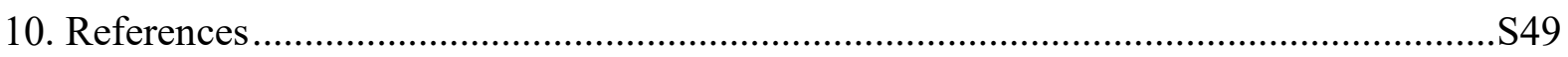




\section{Experimental section}

General Procedures. All reagents were purchased from Sigma-Aldrich, Fisher, Acros, and Alfa Aesar, and were used without further purification. Compound 9 shown in Scheme 1 was prepared according to reported methods. ${ }^{1}$ Column chromatography was performed on basic $\mathrm{Al}_{2} \mathrm{O}_{3}$ (Brockman I, activity, $58 \AA$ ) or $\mathrm{SiO}_{2}(\mathrm{VWR}, 40-60 \mu \mathrm{m}, 60 \AA$ ) and the separated products were monitored by thin layered chromatography (TLC) or by ESI-MS spectrometry. NMR spectra data were collected on a $400-\mathrm{MHz}$ Varian NMR spectrometer or a $600 \mathrm{MHz} \mathrm{MHz}$ Bruker NMR spectrometer in $\mathrm{CDCl}_{3}$ or $d_{6}$-DMSO with TMS as the references. All these ${ }^{13} \mathrm{C}$ NMR spectra were collected by DEPTQ ${ }^{13} \mathrm{C}-\mathrm{NMR}$ spectrometry with CNST12 parameter as $0.5\left(45^{\circ}\right.$ pulse $)$, which provided the quaternary carbon signals as well as the solvent signals in negative phase, and signals from $\mathrm{CH}, \mathrm{CH}_{2}$ and $\mathrm{CH}_{3}$ groups displayed in positive phase. ESITOF-MS and TWIM-MS spectra were collected on a Waters Synapt G2 tandem mass spectrometer, using solutions of $0.01 \mathrm{mg}$ sample in $1 \mathrm{~mL}$ of $\mathrm{CHCl}_{3} / \mathrm{CH}_{3} \mathrm{OH}(1: 3, \mathrm{v} / \mathrm{v})$ for the intermediate compounds and ligands or $1 \mathrm{mg}$ sample in $1 \mathrm{~mL}$ of DMSO/acetonitrile $(1 / 10, \mathrm{v} / \mathrm{v})$ for the complexes.

TWIM-MS. TWIM-MS data were recorded under the following conditions: ESI capillary voltage, $2 \mathrm{kV}$; sample cone voltage, $15 \mathrm{~V}$; extraction cone voltage, $0.1 \mathrm{~V}$; source temperature $100{ }^{\circ} \mathrm{C}$; desolvation temperature, $100{ }^{\circ} \mathrm{C}$; cone gas flow, $10 \mathrm{~L} / \mathrm{h}$; desolvation gas flow, $700 \mathrm{~L} / \mathrm{h}$ $\left(\mathrm{N}_{2}\right)$; source gas control, $0 \mathrm{~mL} / \mathrm{min}$; trap gas control, $2 \mathrm{~mL} / \mathrm{min}$; helium cell gas control, 100 $\mathrm{mL} / \mathrm{min}$; ion mobility (IM) cell gas control, $30 \mathrm{~mL} / \mathrm{min}$; sample flow rate, $5 \mu \mathrm{L} / \mathrm{min}$; IM traveling wave height, $25 \mathrm{~V}$; and IM traveling wave velocity, $1000 \mathrm{~m} / \mathrm{s}$. Q was set in rf-only mode to transmit all ions produced by ESI into the triwave region for the acquisition of TWIMMS data.

Molecular modeling. All the structural optimization and energy calculation were carried out in Materials Studio version 4.2, using the Geometry Optimization and Energy tasks in the Forcite module (Accelrys Software, Inc.). The initial structural model was built up with all counterions omitted for clarity. The Geometry Optimization was performed by using Universal Force Field (UFF) ${ }^{2}$ with atom-based summation and cubic spline truncation for both the electrostatic and Van der Waals parameters. The smart algorithm was used within ultrafine 
calculation quality $\left(2.0 \times 10^{-5} \mathrm{kcal} \cdot \mathrm{mol}^{-1}\right.$ energy, $0.001 \mathrm{kcal} \cdot \mathrm{mol}^{-1} \cdot \AA^{-1}$ Force, $1.0 \times 10^{-5} \AA$ displacement with 50000 iterations for convergence tolerance).

AFM. AFM imaging was performed on a Digital Instrument Nanoscope Dimension 3000 system. The sample was prepared by diluting a DMSO stock solution $(3.0 \mathrm{mg} / \mathrm{mL}, c a .6 .9 \times$ $10^{-5} \mathrm{~mol} / \mathrm{L}$ ) with DMF for 20 times (final con. $c a .3 .5 \times 10^{-6} \mathrm{~mol} / \mathrm{L}$ ); and then dropping the diluted solution onto a freshly cleaved mica surface for $1 \mathrm{~min}$ of incubation; the surface was rinsed with DI water for 5 8 times, and then dried with $\mathrm{N}_{2}$. Silicon cantilevers with ultra-sharp tip (radius $\sim 1-2 \mathrm{~nm}$ ) was used for the experiments.

TEM. The sample solution was diluted by DMF to $3.5 \times 10^{-6} \mathrm{~mol} / \mathrm{L}$. The diluted solution was then drop-casted on to an ultrathin carbon film coated $\mathrm{Cu}$ grid with lacey carbon supports (400 mesh, purchased from Ted Pella, Inc.). The extra solution was absorbed by filter paper to avoid further aggregation. The TEM images were taken with a FEI Morgagni transmission electron microscope.

STM. The sample was dissolved in DMF at a concentration of $5.0 \mathrm{mg} / \mathrm{mL}$ (ca. $1.2 \times 10^{-4}$ mol/L). Solution $(5 \mu \mathrm{L})$ was dropped on a HOPG surface. After 30 seconds, surface was washed slightly with water for three times and totally dried in R.T. in air. The STM images were taken with a Pico Plus SPM system with a PicoScan 3000 Controller. The obtained STM images were processed by WSxM software. ${ }^{3}$

SAXS. HP1 was dissolved in acetonitrile at a concentration of $10 \mathrm{mg} / \mathrm{mL}$ (ca. $2 \times 10^{-4}$ $\mathrm{mol} / \mathrm{L}$ ) and the solution was loaded in a sample cell sandwiched by two mica windows with a gap of $2 \mathrm{~mm}$. The scattering images were acquired with $1 \mathrm{~s}$ of exposure time for twice and averaged to yield the $2 \mathrm{D}$ raw data, which were then circularly averaged and reduced to 1-D profiles as a function of scattering vector, $q$ defined as $\frac{4 \pi}{\lambda} \sin \frac{\theta}{2}$, where $\theta$ is the scattering angle and $\lambda$ is the wavelength. The data cover a $q$ range from 0.004 to $0.7 \AA^{-1}$. SASView 4.1 .2 (https://www.sasview.org/) was used to analyze the 1-D scattering pattern.

The form factor $P(q)$ of hollow cylinder ${ }^{4}$ can be expressed below

$$
\begin{aligned}
& P(q)=\int_{0}^{1} \Psi^{2}\left[q_{z}, R_{\text {outer }}\left(1-x^{2}\right)^{\frac{1}{2}}, R_{\text {core }}\left(1-x^{2}\right)^{\frac{1}{2}}\right]\left[\frac{\sin (q H x)}{q H x}\right]^{2} d x \\
& \Psi[q, a, b]=\frac{2}{R_{\text {outer }}{ }^{2}-R_{\text {core }}{ }^{2}}\left[\frac{R_{\text {outer }}{ }^{2} J_{1}(q a)}{q a}-\frac{R_{\text {core }} J_{1}(q b)}{q b}\right]
\end{aligned}
$$




$$
V_{\text {shell }}=\pi\left(R_{\text {outer }}^{2}-R_{\text {core }}^{2}\right) L
$$

where $\mathrm{H}$ is a half of the cylindrical length and $J_{1}(\mathrm{a})$ is the Bessel function of the first kind and the first order. $R_{\text {outer }}$ and $R_{\text {core }}$ are the outer and core radii, respectively. The scattering intensity, $I_{H C y l}(q)$ can be expressed as

$$
I_{H C y l}(q)=\phi \Delta \rho^{2} P(q)
$$

where $\phi$ and $\Delta \rho$ are the volume fraction of the hollow cylinder and the electron density difference between the cylindrical shell and solvent, respectively.

The Gaussian peak model used to describe the scattering from the stacking between unimers of HP1 inside large scale aggregations is illustrated as the following equations:

$$
I_{\text {Guass }}(q)=\mathrm{A} \exp \left[-\frac{1}{2} \frac{\left(q-q^{*}\right)^{2}}{\sigma^{2}}\right]+\text { background }
$$

Here, the peak has an intensity of A, located at the position of $q^{*}$ with a standard deviation of $\sigma$ for the width of the peak.

\begin{tabular}{|c|c|}
\hline \multicolumn{2}{|c|}{ Hollow cylinder model } \\
\hline $\operatorname{Radius}(\AA)$ & $19.8 \pm 2.5$ \\
\hline Thickness ( & $11.8 \pm 3.0$ \\
\hline $\begin{array}{l}\text { Radius of the base face }(\AA) \\
\text { (Radius + Thickness) }\end{array}$ & $31.5 \pm 5.5$ \\
\hline Height $(\AA)$ of unminer & $25.2 \pm 2.0$ \\
\hline Length $(\AA)$ of aggregation & $>1000$ \\
\hline \multicolumn{2}{|c|}{ Gaussian peak model } \\
\hline Position of peak $1 q^{*}$ & 0.15 \\
\hline Position of peak $2 q^{*}$ & $0.31 \pm 0.002$ \\
\hline Position of peak $3 q^{*}$ & $0.45 \pm 0.003$ \\
\hline
\end{tabular}

Table S1. Structural parameters determined by SAXS model fitting analysis for a unimer and aggregations of HP1 in acetonitrile solution.

3D deconvolution fluorescence microscopy. A $100 \mu \mathrm{L}$ aliquot of cultures, of midlogarithmic phase $S$. aureus cells and B. Subtilus cells grown in tryptic soy broth medium, were incubated with supramolecules HP1 and HP2 at indicated concentrations for $5 \mathrm{~min}$ at room 
temperature. $5 \mu \mathrm{L}$ was spotted on a glass bottom culture dish (MatTek) and covered with a 1\% agarose pad made with distilled water. Cells were viewed at room temperature with a DeltaVision Elite microscope system (Applied Precision/GE Healthcare) equipped with a Photometrics CoolSnap HQ2 camera and an environmental chamber. HP1 and HP2 were captured with TRITC and DAPI filters respectively. 17 planes were acquired every $200 \mathrm{~nm}$, and the data were analyzed using manufacturer-provided SoftWorx software.

Antimicrobial activity against MRSA and B. subtilus. ${ }^{5}$ Briefly, a single colony of MRSA (or B. subtills) bacterium was incubated in $3 \mathrm{~mL}$ TSB medium and allowed to grow overnight at $37^{\circ} \mathrm{C}$. The bacteria culture was then diluted at $1: 100$ and the bacteria were able to re-grow to mid-logarithmic phase in 6-8 h. Next, $50 \mu \mathrm{L}$ compounds in 2 -fold serially diluted solution with the concentrations of $0.39-25 \mu \mathrm{g} / \mathrm{mL}$ were added to the $96-w e l l$ plate containing $50 \mu \mathrm{L}$ of bacteria suspension $\left(1 \times 10^{6} \mathrm{CFU} / \mathrm{mL}\right)$ in each well. Following that, the plate was incubated at $37^{\circ} \mathrm{C}$ for $16 \mathrm{~h}$, and the absorption of those wells at $600 \mathrm{~nm}$ wavelength was read on a Biotek Synergy $\mathrm{H} 1$ microtiter plate reader. The $\mathrm{IC}_{50} \mathrm{~s}$ were determined as the concentrations of compounds that inhibit half of the growth of MRSA or B. subtills. The experiments were repeated at least three times with duplicates each time.

Hemolytic assay. ${ }^{5}$ The freshly drawn human red blood cells (hRBCs) were washed with $1 \times$ PBS buffer multiple times and centrifuged at $3500 \mathrm{rpm}$ for $10 \mathrm{~min}$, until the supernatant became clear. Next, the supernatant was removed, and the RBCs were diluted into $5 \% \mathrm{v} / \mathrm{v}$ suspension. The aliquots of suspension $(50 \mu \mathrm{L})$ were then incubated with compounds at various concentrations in 2 -fold serially diluted solution $(50 \mu \mathrm{L})$ at $37{ }^{\circ} \mathrm{C}$ for $1 \mathrm{~h}$. The mixture was centrifuged at $3500 \mathrm{rpm}$ for $10 \mathrm{~min}$ and the supernatant was collected. Following that, $100 \mu \mathrm{L}$ PBS was added to $30 \mu \mathrm{L}$ of the supernatant, and the absorbance of the solution at $540 \mathrm{~nm}$ was read on a Biotek Synergy H1 plate reader. 2\% Triton X-100 was used as the positive control and $1 \times$ PBS was used the negative control. The hemolysis activity was calculated by the formula $\%$ hemolysis $=\left(\mathrm{Abs}_{\text {sample}}-\mathrm{AbSPBS}\right) /\left(\mathrm{Abs}_{\text {Triton }}-\mathrm{AbSPBS}\right) \times 100 \%$. The experiment was repeated at least three times with duplicates each time. 


\section{Synthesis of the Ligands L1 and L2}<smiles>CCCCCCCOc1ccc(Br)cc1C=O</smiles>

Compound 1:6 2-hydroxyl-5-bromo benzaldehyde (5.00 g, $25.0 \mathrm{mmol})$, 1-bromohexane (4.50 g, $27.5 \mathrm{mmol})$, and $\mathrm{K}_{2} \mathrm{CO}_{3}(6.90 \mathrm{~g}, 50.0 \mathrm{mmol})$ were suspended in DMF $(25 \mathrm{~mL})$. The mixture was heated overnight under $\mathrm{N}_{2}$ atmosphere. After cooling to room temperature, the solvent was removed under reduced pressure, and the residue was redissolved in ethyl acetate. The organic phase was washed with deionized (DI) water, brine, and dried by anhydrous $\mathrm{Na}_{2} \mathrm{SO}_{4}$. The solvent was removed by rotatory evaporator, and the residue was separated by a silica gel column chromatography with dichloromethane (DCM)/hexane $(1 / 4, \mathrm{v} / \mathrm{v})$ to afford the product as colorless oil $\left(6.50 \mathrm{~g}, 92 \%\right.$ yield) ${ }^{1} \mathrm{H}$ NMR $\left(400 \mathrm{MHz}, \mathrm{CDCl}_{3}, 300 \mathrm{~K}\right) \delta(\mathrm{ppm}) 10.42(\mathrm{~s}, 1 \mathrm{H}$, -CHO), $7.92\left(\mathrm{~d}, J=2.6 \mathrm{~Hz}, 1 \mathrm{H}, \mathrm{Ph}-H^{\mathrm{A}}\right), 7.60\left(\mathrm{dd}, J=8.9,2.6 \mathrm{~Hz}, 1 \mathrm{H}, \mathrm{Ph}-H^{\mathrm{C}}\right), 6.88(\mathrm{~d}, J=8.9$ $\left.\mathrm{Hz}, 1 \mathrm{H}, \mathrm{Ph}-H^{\mathrm{B}}\right), 4.06(\mathrm{t}, J=6.4 \mathrm{~Hz}, 2 \mathrm{H}), 1.93-1.78(\mathrm{~m}, 2 \mathrm{H}), 1.52-1.42(\mathrm{~m}, 2 \mathrm{H}), 1.35(\mathrm{~m}, J$ $=7.1,3.6 \mathrm{~Hz}, 4 \mathrm{H}), 0.91(\mathrm{t}, J=7.0 \mathrm{~Hz}, 3 \mathrm{H})$.<smiles>CCCOc1ccc(Br)cc1C=O</smiles>

Compound 2:6 Similar synthetic method of Compound 1 was applied in synthesis of Compound 2. $4.00 \mathrm{~g}$ 2-hydroxyl-5-bromo benzaldehyde $(20.0 \mathrm{mmol})$ yielded $7.20 \mathrm{~g}$ white solid (97\%). ${ }^{1} \mathrm{H}$ NMR (400 MHz, $\left.\mathrm{CDCl}_{3}, 300 \mathrm{~K}\right) \delta(\mathrm{ppm}) 10.40$ (s, 1H, -CHO), 7.90 (d, J=2.6 Hz, $\left.1 \mathrm{H}, \mathrm{Ph}-H^{\mathrm{A}}\right), 7.58\left(\mathrm{dd}, J=8.9,2.6 \mathrm{~Hz}, 1 \mathrm{H}, \mathrm{Ph}-H^{\mathrm{C}}\right), 6.86\left(\mathrm{~d}, J=8.9 \mathrm{~Hz}, 1 \mathrm{H}, \mathrm{Ph}-H^{\mathrm{B}}\right), 4.04(\mathrm{t}, J$ $=6.4 \mathrm{~Hz}, 2 \mathrm{H}), 1.88-1.75(\mathrm{~m}, 2 \mathrm{H}), 1.49-1.40(\mathrm{~m}, 2 \mathrm{H}), 1.35-1.21(\mathrm{~m}, 16 \mathrm{H}), 0.86(\mathrm{t}, J=6.8$ $\mathrm{Hz}, 3 \mathrm{H})$.

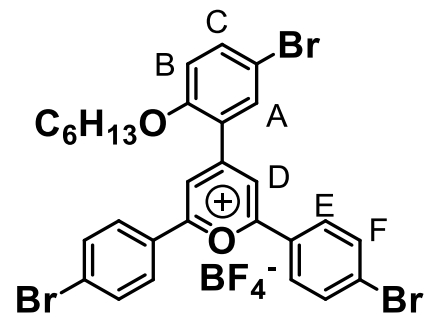

Compound 3: To a $100 \mathrm{~mL}$ Schlenk flask, Compound 1 (2.80 g, $10.0 \mathrm{~mol})$ and 4'- 
bromoacetophenone $(4.00 \mathrm{~g}, 20.0 \mathrm{mmol})$ were added. Under $\mathrm{N}_{2}$ atmosphere, $\mathrm{BF}_{3} \cdot \mathrm{OEt}_{2}(5.0$ $\mathrm{mL}, 40 \mathrm{~mol}$ ) was added and the mixture was stirred at $100^{\circ} \mathrm{C}$ for 4 hours. After cooling to room temperature, $10 \mathrm{~mL}$ acetone was added to dissolve the obtained solid. The resultant mixture was poured into $300 \mathrm{~mL}$ ether to form precipitate. The precipitate was collected by filtration, repeatedly washed with fresh ether, and dried under vacuum. $3.00 \mathrm{~g}$ yellow solid was obtained with yield as $41 \%$. The Compound 3 was directly used for the next step reaction but without further purification. ${ }^{1} \mathrm{H}$ NMR (400 MHz, $d_{6}$-DMSO, 298K) $\delta(\mathrm{ppm}) 9.11\left(\mathrm{~s}, 2 \mathrm{H}, \mathrm{Py}-H^{\mathrm{D}}\right), 8.42$ $\left(\mathrm{d}, \mathrm{J}=8.7 \mathrm{~Hz}, 4 \mathrm{H}, \mathrm{Ph}-H^{\mathrm{E}}\right), 8.30\left(\mathrm{~d}, \mathrm{~J}=2.4 \mathrm{~Hz}, 1 \mathrm{H}, \mathrm{Ph}-H^{\mathrm{A}}\right), 8.00\left(\mathrm{~d}, \mathrm{~J}=8.7 \mathrm{~Hz}, 4 \mathrm{H}, \mathrm{Ph}-H^{\mathrm{F}}\right)$, $7.90\left(\mathrm{dd}, \mathrm{J}=9.1,2.4 \mathrm{~Hz}, 1 \mathrm{H}, \mathrm{Ph}-H^{\mathrm{C}}\right), 7.35\left(\mathrm{~d}, \mathrm{~J}=9.1 \mathrm{~Hz}, 1 \mathrm{H}, \mathrm{Ph}-H^{\mathrm{B}}\right), 4.19(\mathrm{t}, \mathrm{J}=6.2 \mathrm{~Hz}, 2 \mathrm{H})$, $1.86-1.68(\mathrm{~m}, 2 \mathrm{H}), 1.42-1.29(\mathrm{~m}, 2 \mathrm{H}), 1.19(\mathrm{~m}, 2 \mathrm{H}), 1.07(\mathrm{~m}, 2 \mathrm{H}), 0.73(\mathrm{t}, \mathrm{J}=7.3 \mathrm{~Hz}, 3 \mathrm{H})$. DEPTQ ${ }^{13} \mathrm{C}$ NMR $\left(125 \mathrm{MHz}, d_{6}\right.$-DMSO, $\left.300 \mathrm{~K}\right) \delta(\mathrm{ppm}) 168.82,163.33,157.40,138.24$, $133.75,132.97,131.73,130.42,129.51,128.28,124.10,118.86,115.82,112.78,69.33,31.14$, 28.57, 25.42, 21.93, 13.83. ESI-MS $(\mathrm{m} / \mathrm{z})$ : calcd. for $\left[\mathrm{M}-\mathrm{BF}_{4}^{-}\right]^{+} 642.95$, found 642.96

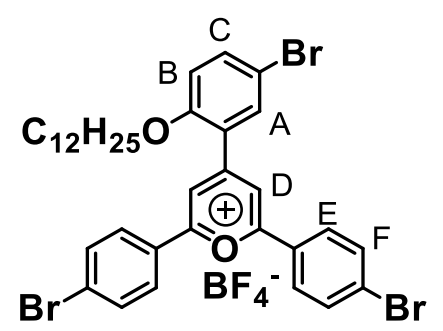

Compound 4: Similar synthetic method of Compound 3 was applied in the synthesis of Compound 4. $1.76 \mathrm{~g}$ Compound $2(5.0 \mathrm{mmol})$ afforded $1.35 \mathrm{~g}$ orange powder with yield as 37\%. ${ }^{1} \mathrm{H}$ NMR (400 MHz, d6-DMSO, 298K) $\delta$ (ppm) 9.12 (s, 2H, Py- $H^{\mathrm{D}}$ ), 8.43 (d, J = $8.7 \mathrm{~Hz}$, 4H, Ph- $\left.H^{\mathrm{E}}\right), 8.30\left(\mathrm{~d}, \mathrm{~J}=2.3 \mathrm{~Hz}, 1 \mathrm{H}, \mathrm{Ph}-H^{\mathrm{A}}\right), 7.99\left(\mathrm{~d}, \mathrm{~J}=8.7 \mathrm{~Hz}, 4 \mathrm{H}, \mathrm{Ph}-H^{\mathrm{F}}\right), 7.91(\mathrm{dd}, \mathrm{J}=9.1$, $\left.2.3 \mathrm{~Hz}, 1 \mathrm{H}, \mathrm{Ph}-H^{\mathrm{C}}\right), 7.35\left(\mathrm{~d}, \mathrm{~J}=9.1 \mathrm{~Hz}, 1 \mathrm{H}, \mathrm{Ph}-H^{\mathrm{B}}\right), 4.19(\mathrm{t}, \mathrm{J}=6.0 \mathrm{~Hz}, 2 \mathrm{H}), 1.78-1.68(\mathrm{~m}$, 2H), $1.40-1.32(\mathrm{~m}, 2 \mathrm{H}), 1.28-0.95(\mathrm{~m}, 19 \mathrm{H}), 0.85(\mathrm{t}, \mathrm{J}=7.0 \mathrm{~Hz}, 3 \mathrm{H})$. DEPTQ ${ }^{13} \mathrm{C}$ NMR (125 MHz, d6-DMSO, 298K) $\delta(\mathrm{ppm}) 168.76,163.38,157.39,138.23,133.72,132.95,130.40$, $129.54,128.22,124.11,118.84,115.79,112.77,69.33,31.30,29.10,29.10,29.06,29.04,28.97$, 28.72, 28.63, 25.89, 22.11, 13.95. ESI-MS $(\mathrm{m} / \mathrm{z})$ : calcd. for $\left[\mathrm{M}-\mathrm{BF}_{4}^{-}\right]^{+} 727.04$, found 727.05 


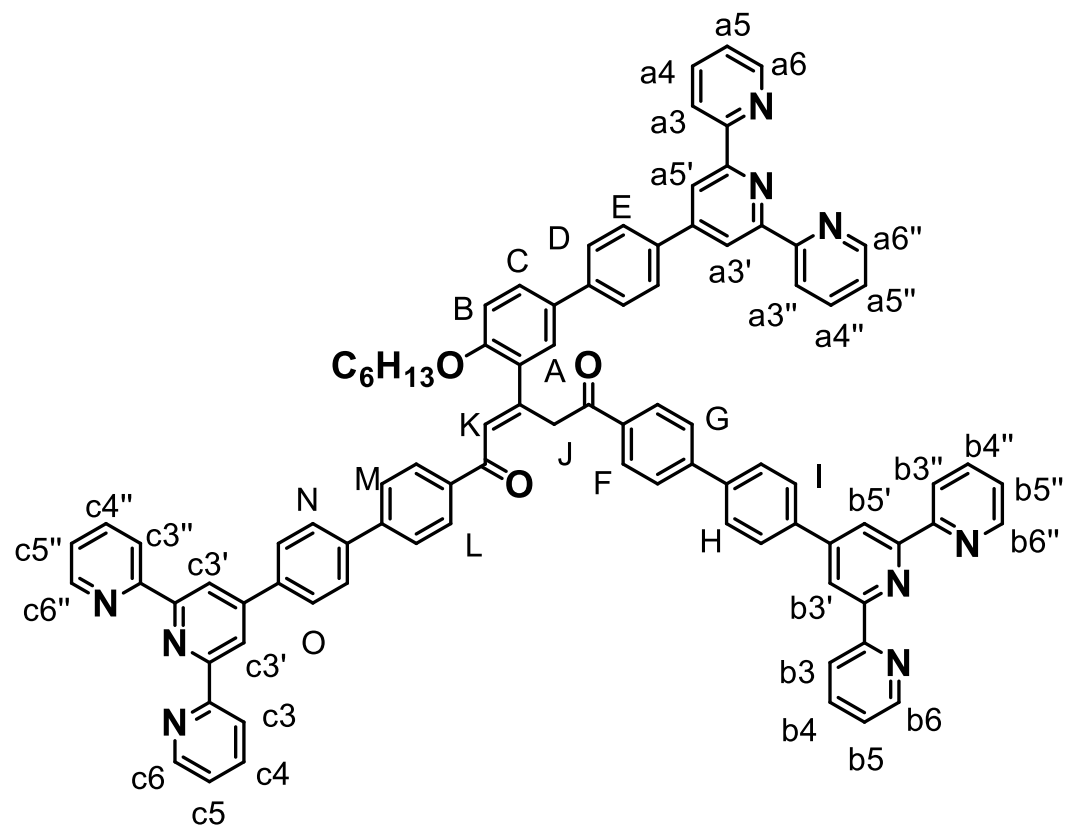

Compound 5: Compound 3 (0.98 g, 1.3 mmol), (4-([2,2':6',2"-terpyridin]-4'-yl)phenyl)boronic $\operatorname{acids}^{7}(2.75 \mathrm{~g}, 7.80 \mathrm{mmol}), \mathrm{Pd}\left(\mathrm{PPh}_{3}\right)_{4}(225 \mathrm{mg}, 0.195 \mathrm{mmol})$ and $\mathrm{NaHCO}_{3}(1.64 \mathrm{~g}, 20.0 \mathrm{mmol})$ were added into a $200 \mathrm{~mL}$ Schlenk flask. Under $\mathrm{N}_{2}$ atmosphere, dioxane $(60 \mathrm{~mL})$ and water $(20 \mathrm{~mL})$ were added. The mixture was heated to $75{ }^{\circ} \mathrm{C}$ and kept stirring for 6 hours. After cooling down to room temperature, the solvent was evaporated. The residue was dissolved in DCM, and then washed with water for 3 times. The organic layer was collected and dried over anhydrous $\mathrm{MgSO}_{4}$. After purification by $\mathrm{Al}_{2} \mathrm{O}_{3}$ column with $\mathrm{DCM} / \mathrm{CH}_{3} \mathrm{OH}(100 / 0.4$, v/v) as the eluent, Compound 5 (1.38 g, 65\%) was obtained as yellow powder. ${ }^{1} \mathrm{H}$ NMR (400 MHz, $\left.\mathrm{CDCl}_{3}, 298 \mathrm{~K}\right) \delta(\mathrm{ppm}) 8.79\left(\mathrm{~s}, 6 \mathrm{H}, \mathrm{TPY}-H^{\mathrm{a}-\mathrm{c} 3^{\prime}, 5^{\prime}}\right), 8.74\left(\mathrm{~m}, 6 \mathrm{H}, \mathrm{TPY}-H^{\mathrm{a}-\mathrm{c} 6,6^{\prime}}\right), 8.72-8.64(\mathrm{~m}$, 6H, TPY- $\left.H^{\mathrm{a}-\mathrm{c} 3,3 ”}\right), 8.16\left(\mathrm{~m}, 3 \mathrm{H}, \mathrm{Ph}-H^{\mathrm{I}, \mathrm{O}}\right), 8.08-7.97\left(\mathrm{~m}, 6 \mathrm{H}, \mathrm{Ph}-H^{\mathrm{E}, \mathrm{F}, \mathrm{L}}\right), 7.93-7.84(\mathrm{~m}, 6 \mathrm{H}$, TPY- $\left.H^{\mathrm{a}-\mathrm{c} 4,4 ”}\right), 7.85-7.72\left(\mathrm{~m}, 12 \mathrm{H}, \mathrm{Ph}-H^{\mathrm{A}, \mathrm{D}, \mathrm{G}, \mathrm{H}, \mathrm{M}, \mathrm{N}}\right), 7.66\left(\mathrm{dd}, \mathrm{J}=8.6,2.3 \mathrm{~Hz}, 1 \mathrm{H}, \mathrm{Ph}-H^{\mathrm{C}}\right), 7.46$ (s, 1H, Ph- $\left.H^{\mathrm{K}}\right), 7.35\left(\mathrm{~m}, 6 \mathrm{H}, \mathrm{TPY}-H^{\mathrm{a}-\mathrm{c} 5,5 ”}\right), 7.03\left(\mathrm{~d}, \mathrm{~J}=8.6 \mathrm{~Hz}, 1 \mathrm{H}, \mathrm{Ph}-H^{\mathrm{B}}\right), 4.99\left(\mathrm{~s}, 2 \mathrm{H}, H^{\mathrm{J}}\right)$, 4.07 (t, J = 6.5 Hz, 2H), $1.85-1.70(\mathrm{~m}, 2 \mathrm{H}), 1.45(\mathrm{~m}, 2 \mathrm{H}), 1.37-1.20(\mathrm{~m}, 4 \mathrm{H}), 0.89$ (d, J = $6.8 \mathrm{~Hz}, 3 \mathrm{H})$. DEPTQ ${ }^{13} \mathrm{C}$ NMR (125 MHz, $\left.\mathrm{CDCl}_{3}, 298 \mathrm{~K}\right) \delta(\mathrm{ppm}) 195.13,190.42,156.26$, $156.18,156.07,155.99,155.94,152.41,149.72,149.50,149.13,144.58,144.43,140.92$, $140.64,140.56,138.12,138.02,136.84,136.81,136.43,132.91,132.31,129.32,129.12$, $129.03,128.89,128.71,128.60,127.85,127.83,127.75,127.73,127.21,127.15,125.89$, $123.83,123.77,121.35,118.69,118.62,112.23,68.72,43.66,31.57,29.19,25.94,22.58,14.06$. HR-ESI-MS $(\mathrm{m} / \mathrm{z})$ : calcd. for $[\mathrm{M}+2 \mathrm{H}]^{2+} 674.7840$ (found 674.7851 ); calcd. for $[\mathrm{M}+3 \mathrm{H}]^{3+}$ 
450.1919 (found 450.1927).

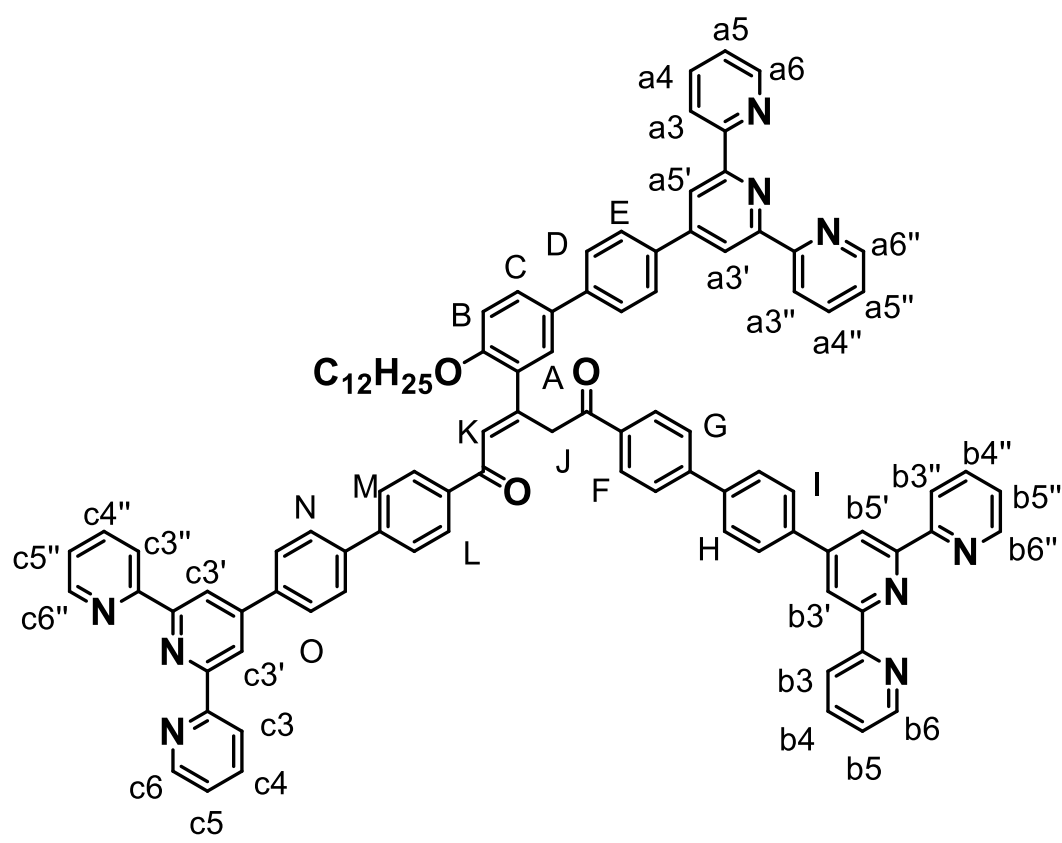

Compound 6: Compound 6 was synthesized using the same method of preparing Compound 5. $1.64 \mathrm{~g}$ Compound 4 (2.0 mmol) afforded $1.79 \mathrm{~g}$ Compound 6 as yellow powder (yield: $62 \%$ ). ${ }^{1} \mathrm{H}$ NMR $\left(400 \mathrm{MHz}, \mathrm{CDCl}_{3}, 298 \mathrm{~K}\right) \delta(\mathrm{ppm}) 8.79$ (s, 6H, TPY- $\left.H^{\mathrm{a}-\mathrm{c} 3^{\prime}, 5^{\prime}}\right), 8.74$ (d, J = 4.0 Hz, 6H, TPY- $\left.H^{\mathrm{a}-\mathrm{c} 6,6 ”}\right), 8.71-8.64\left(\mathrm{~m}, 6 \mathrm{H}, \mathrm{TPY}-H^{\mathrm{a}-\mathrm{c} 3,3 ”}\right), 8.16\left(\mathrm{~m}, 3 \mathrm{H}, \mathrm{Ph}-H^{\mathrm{I}, \mathrm{O}}\right), 8.07-7.97(\mathrm{~m}, 6 \mathrm{H}, \mathrm{Ph}-$ $\left.H^{\mathrm{E}, \mathrm{F}, \mathrm{L}}\right), 7.93-7.84\left(\mathrm{~m}, 6 \mathrm{H}, \mathrm{TPY}-H^{\mathrm{a}-\mathrm{c} 4,4 "}\right), 7.83-7.71\left(\mathrm{~m}, 12 \mathrm{H}, \mathrm{Ph}-H^{\mathrm{A}, \mathrm{D}, \mathrm{G}, \mathrm{H}, \mathrm{M}, \mathrm{N}}\right), 7.66(\mathrm{dd}, \mathrm{J}=$ 8.6, 2.3 Hz, 1H Ph- $\left.H^{\mathrm{C}}\right), 7.46\left(\mathrm{~s}, 1 \mathrm{H}, \mathrm{Ph}-H^{\mathrm{K}}\right), 7.35$ (m, 6H, TPY- $\left.H^{\mathrm{a}-\mathrm{c} 5,5 ”}\right), 7.03$ (d, J = $8.6 \mathrm{~Hz}$, $\left.1 \mathrm{H}, \mathrm{Ph}-H^{\mathrm{B}}\right), 4.99\left(\mathrm{~s}, 2 \mathrm{H}, H^{\mathrm{J}}\right), 4.06(\mathrm{t}, \mathrm{J}=6.5 \mathrm{~Hz}, 2 \mathrm{H}), 1.86-1.66(\mathrm{~m}, 2 \mathrm{H}), 1.44(\mathrm{~s}, 2 \mathrm{H}), 1.22$ $(\mathrm{m}, 16 \mathrm{H}), 0.83(\mathrm{t}, \mathrm{J}=6.7 \mathrm{~Hz}, 3 \mathrm{H})$. DEPTQ ${ }^{13} \mathrm{C} \mathrm{NMR}\left(125 \mathrm{MHz}, \mathrm{CDCl}_{3}, 298 \mathrm{~K}\right) \delta(\mathrm{ppm}) 195.16$, $190.41,156.28,156.20,156.07,156.01,155.95,152.46,149.74,149.52,149.14,144.57$, $144.44,140.93,140.64,140.58,138.13,138.02,136.85,136.44,132.92,132.33,129.13$, $128.90,128.73,128.60,127.85,127.75,127.22,127.16,125.88,123.83,123.78,121.35$, $118.70,118.64,112.24,68.73,43.68,31.89,29.71,29.67,29.66,29.65,29.46,29.36,29.24$, 26.32, 22.67, 14.09. HR-ESI-MS (m/z): calcd. for $[\mathrm{M}+2 \mathrm{H}]^{2+} 716.8309$ (found 716.8301); calcd. for $[\mathrm{M}+3 \mathrm{H}]^{3+} 478.2232$ (found 478.2246). 


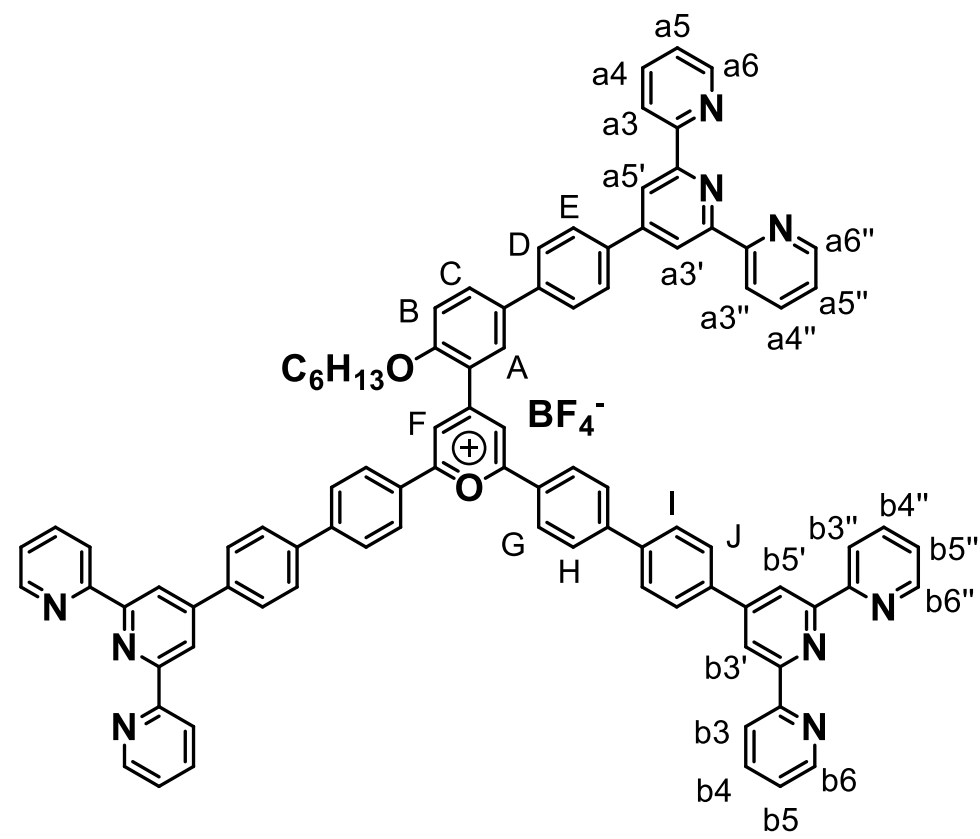

Compound 7: Compound $5(0.67 \mathrm{~g}, 0.50 \mathrm{mmol})$ was dissolved in a mixture of $\mathrm{CHCl}_{3}(80 \mathrm{~mL})$ and $\mathrm{CH}_{3} \mathrm{OH}(80 \mathrm{~mL})$. The suspension was kept stirring at room temperature until a clear solution was formed. $\mathrm{HBF}_{4}(1 \mathrm{~mL}, 50$ wt. \% in water, diluted by additional $5 \mathrm{~mL} \mathrm{CH} 3 \mathrm{OH}$, excess amount) was added dropwise to this clear solution, then kept stirring at room temperature overnight. The precipitate was collected by centrifugation and washed repeatedly with DCM and then water. After drying in vacuum, compound 7 was obtained as a dark red solid (0.68 g, 96\%). ${ }^{1} \mathrm{H}$ NMR (400 MHz, $d_{6}$-DMSO, 298K) $\delta$ (ppm) 9.17 (s, 2H, Py- $H^{\mathrm{F}}$ ), 8.77

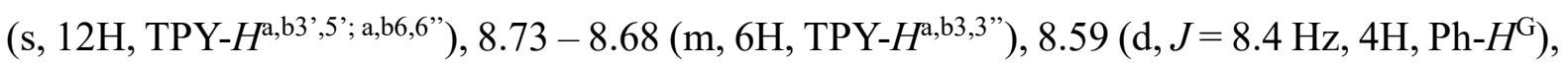
$8.38\left(\mathrm{~s}, 1 \mathrm{H}, \mathrm{Ph}-H^{\mathrm{A}}\right), 8.17$ (d, $\left.J=8.4 \mathrm{~Hz}, 4 \mathrm{H}, \mathrm{Ph}-H^{\mathrm{H}}\right), 8.14-7.98$ (m, 19H, TPY- $H^{\mathrm{a}, \mathrm{b} 4,4}$ ", Ph$\left.H^{\mathrm{C}, \mathrm{D}, \mathrm{E}, \mathrm{IJ} J}\right), 7.58\left(\mathrm{~m}, 6 \mathrm{H}\right.$, , TPY- $\left.H^{\mathrm{a}, \mathrm{b} 5,5 ”}\right), 7.50\left(\mathrm{~d}, J=8.8 \mathrm{~Hz}, 1 \mathrm{H}, \mathrm{Ph}-H^{\mathrm{B}}\right), 4.29(\mathrm{t}, J=5.6 \mathrm{~Hz}, 2 \mathrm{H})$, $1.85(\mathrm{~m}, 2 \mathrm{H}), 1.47(\mathrm{~m}, 2 \mathrm{H}), 1.34-1.20(\mathrm{~m}, 2 \mathrm{H}), 1.15(\mathrm{~m}, 2 \mathrm{H}), 0.74(\mathrm{t}, J=7.2 \mathrm{~Hz}, 3 \mathrm{H})$. DEPTQ ${ }^{13} \mathrm{C}$ NMR (125 MHz, $d_{6}$-DMSO, 298K) $\delta(\mathrm{ppm}) 168.25,163.80,157.98,153.73,152.90$, $152.67,149.24,148.84,148.77,147.81,145.07,140.34,139.63,138.87,137.14,135.74$, $134.61,132.46,129.09,127.90,127.77,127.73,127.55,127.43,125.54,125.29,122.00$, $118.71,114.25,69.42,31.36,28.98,25.59,22.13,14.00$. HR ESI-MS $(\mathrm{m} / \mathrm{z})$ : calcd. for $\left[\mathrm{M}-\mathrm{BF}_{4}^{-}\right]^{+} 1330.5496$ (found 1330.5487), calcd. for $\left[\mathrm{M}-\mathrm{BF}_{4}{ }^{-}+\mathrm{H}\right]^{2+} 665.7787$ (found 665.7780). 


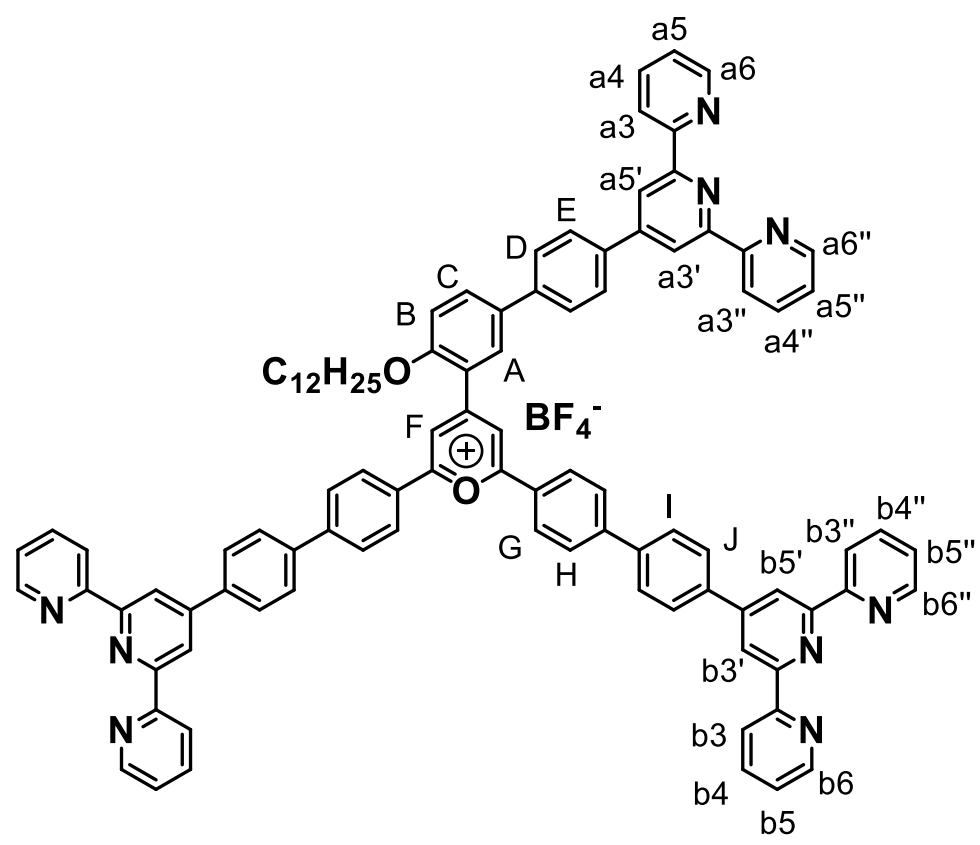

Compound 8: Compound 8 was prepared using the same procedure of synthesizing Compound 7. $930 \mathrm{mg}$ Compound 6 (0.65 mmol) afforded $870 \mathrm{mg}$ dark red powder (yield: $90 \%) .{ }^{1} \mathrm{H}$ NMR (400 MHz, $d_{6}$-DMSO, 298K) $\delta(\mathrm{ppm}) 9.14$ (s, 2H, Py- $\left.H^{\mathrm{F}}\right), 8.76$ (s, 12H, TPY- $\left.H^{\mathrm{a}, \mathrm{b} 3^{\prime}, 5^{\prime} ; \mathrm{a}, \mathrm{b} 6,6^{\prime}}\right)$, $8.72\left(\mathrm{~m}, 6 \mathrm{H}, \mathrm{TPY}-H^{\mathrm{a}, \mathrm{b3}, 3 ”}\right), 8.57\left(\mathrm{~d}, J=8.3 \mathrm{~Hz}, 4 \mathrm{H}, \mathrm{Ph}-H^{\mathrm{G}}\right), 8.35\left(\mathrm{~s}, 1 \mathrm{H}, \mathrm{Ph}-H^{\mathrm{A}}\right), 8.14(\mathrm{~d}, J=$ $\left.8.4 \mathrm{~Hz}, 4 \mathrm{H}, \mathrm{Ph}-H^{\mathrm{H}}\right), 8.13-8.04\left(\mathrm{~m}, 16 \mathrm{H}, \mathrm{TPY}-H^{\mathrm{a}, \mathrm{b} 4,4}\right.$,, $\left.\mathrm{Ph}-H^{\mathrm{E}, \mathrm{I}, \mathrm{J}}\right), 8.03(\mathrm{~d}, J=4.3 \mathrm{~Hz}, 2 \mathrm{H}, \mathrm{Ph}-$ $\left.H^{\mathrm{D}}\right), 8.01\left(\mathrm{~s}, 1 \mathrm{H}, \mathrm{Ph}-H^{\mathrm{C}}\right), 7.62-7.56\left(\mathrm{~m}, 6 \mathrm{H}, \mathrm{TPY}-H^{\mathrm{a}, \mathrm{b} 5,5 "}\right), 7.46\left(\mathrm{~d}, J=9.0 \mathrm{~Hz}, 1 \mathrm{H}, \mathrm{Ph}-H^{\mathrm{C}}\right)$, $4.26(\mathrm{~m}, 2 \mathrm{H}), 1.83(\mathrm{~m}, 2 \mathrm{H}), 1.44(\mathrm{~m}, 2 \mathrm{H}), 1.22(\mathrm{~m}, 2 \mathrm{H}), 1.00(\mathrm{~m}, 14 \mathrm{H}), 0.62(\mathrm{t}, J=6.8 \mathrm{~Hz}$, 3H). DEPTQ ${ }^{13} \mathrm{C}$ NMR (125 MHz, $d_{6}$-DMSO, 298K) $\delta$ (ppm) 168.06, 163.71, 157.91, 152.89, $151.94,151.79,149.35,148.84,147.19,145.01,140.38,139.44,138.73,136.79,135.28$, $134.74,132.48,128.98,127.83,127.77,127.72,127.49,127.34,125.83,125.55,122.34$, $118.98,117.57,114.25,69.55,31.31,29.50,29.46,29.32,29.21,29.20,29.19$, 28.77, 26.13, 22.05, 13.82. HR ESI-MS $(\mathrm{m} / \mathrm{z})$ : calcd. for $\left[\mathrm{M}-\mathrm{BF}_{4}{ }^{-}+\mathrm{H}\right]^{2+} 707.8257$ (found 707.8262), calcd. for $\left[\mathrm{M}-\mathrm{BF}_{4}{ }^{-}+2 \mathrm{H}\right]^{3+} 472.2197$ (found 472.2208). 


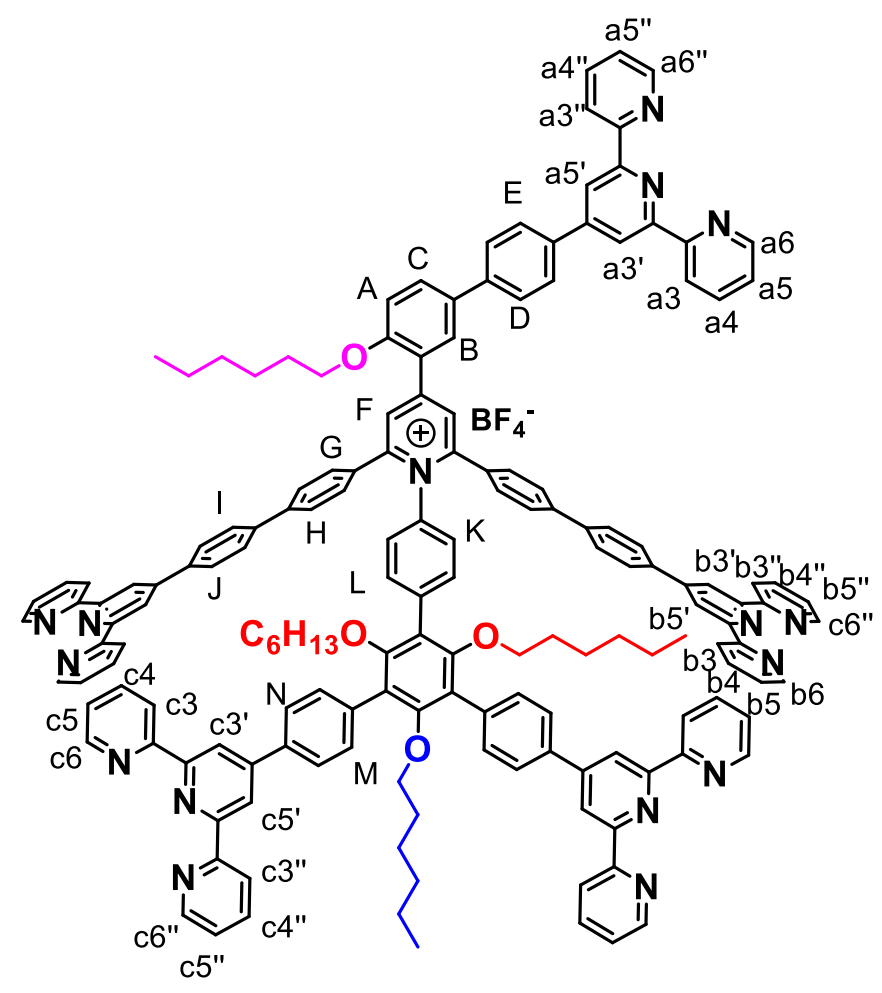

Ligand L1: A mixture of Compound 7 (339 mg, 0.239 mmol), Compound 9 (216 mg, 0.200 $\mathrm{mmol})$, and molecular sieve $4 \AA$ (30 mg) in DMSO $(20 \mathrm{~mL})$ was stirred at $120^{\circ} \mathrm{C}$ for 36 hours. After cooling down to room temperature, the mixture was dissolved in DCM (100 mL) and washed repeatedly with brine water. The organic layer was collected and dried over anhydrous $\mathrm{MgSO}_{4}$. After removal of the solvent under reduced pressure, the crude product was purified by $\mathrm{Al}_{2} \mathrm{O}_{3}$ column chromatography with $\mathrm{DCM} / \mathrm{CH}_{3} \mathrm{OH}(100 / 0.8, \mathrm{v} / \mathrm{v})$ as the eluent for twice, which gave $\mathbf{L 1}$ as a yellowish powder $(220 \mathrm{mg}, 45 \%) .{ }^{1} \mathrm{H} \mathrm{NMR}\left(600 \mathrm{MHz}, \mathrm{CDCl}_{3}, 300 \mathrm{~K}\right) \delta$ (ppm) $8.78\left(\mathrm{~s}, 2 \mathrm{H}, \mathrm{TPY}-H^{\mathrm{a} 3^{\prime}, 5^{\prime}}\right), 8.75-8.73\left(\mathrm{~m}, 2 \mathrm{H}, \mathrm{TPY}-H^{\mathrm{a} 6,6^{\prime}}\right), 8.73\left(\mathrm{~s}, 4 \mathrm{H}, \mathrm{TPY}-H^{\mathrm{b} 3^{\prime}, 5^{\prime}}\right), 8.71$ (s, 4H, TPY- $\left.H^{\mathrm{c}^{\prime}, 5^{\prime}}\right), 8.67$ (d, $\left.J=7.9 \mathrm{~Hz}, 2 \mathrm{H}, \mathrm{TPY}-H^{\mathrm{a}, 3,3^{\prime}}\right), 8.63$ (m, 16H, TPY- $\left.H^{\mathrm{b}, \mathrm{c} 6,6^{\prime \prime} ; \mathrm{b}, \mathrm{c} 3,3^{\prime \prime}}\right)$, $8.41\left(\mathrm{~s}, 2 \mathrm{H}, \mathrm{Py}-H^{\mathrm{F}}\right), 8.20\left(\mathrm{~d}, J=2.2 \mathrm{~Hz}, 1 \mathrm{H}, \mathrm{Ph}-H^{\mathrm{B}}\right), 8.01\left(\mathrm{~d}, J=8.4 \mathrm{~Hz}, 2 \mathrm{H}, \mathrm{Ph}-H^{\mathrm{E}}\right), 7.97(\mathrm{~d}$, $\left.J=8.7 \mathrm{~Hz}, 2 \mathrm{H}, \mathrm{Ph}-H^{\mathrm{K}}\right), 7.94\left(\mathrm{~d}, J=8.4 \mathrm{~Hz}, 4 \mathrm{H}, \mathrm{Ph}-H^{\mathrm{G}}\right), 7.92-7.88\left(\mathrm{~m}, 8 \mathrm{H}, \mathrm{Ph}-H^{\mathrm{J}, \mathrm{N}}\right), 7.88-$ $7.80\left(\mathrm{~m}, 13 \mathrm{H}, \mathrm{TPY}-H^{\mathrm{a}-\mathrm{c} 4,4^{\prime}}, \mathrm{Ph}-H^{\mathrm{C}, \mathrm{D}}\right), 7.65\left(\mathrm{~m}, 8 \mathrm{H}, \mathrm{Ph}-H^{\mathrm{I}, \mathrm{H}}\right), 7.57\left(\mathrm{~d}, J=8.8 \mathrm{~Hz}, 2 \mathrm{H}, \mathrm{Ph}-H^{\mathrm{L}}\right)$, $7.55\left(\mathrm{~d}, J=8.3 \mathrm{~Hz}, 4 \mathrm{H}, \mathrm{Ph}-H^{\mathrm{M}}\right), 7.35$ (ddd, $\left.J=7.5,4.7,1.1 \mathrm{~Hz}, 2 \mathrm{H}, \mathrm{TPY}-H^{\mathrm{a} 5,5 ”}\right), 7.30-7.26$ (m, 8H, TPY- $\left.H^{\mathrm{b}, \mathrm{c} 5,5 ”}\right), 7.19\left(\mathrm{~d}, J=8.7 \mathrm{~Hz}, 1 \mathrm{H}, \mathrm{Ph}-H^{\mathrm{A}}\right), 4.20\left(\mathrm{t}, J=6.3 \mathrm{~Hz}, 2 \mathrm{H},-\mathrm{CH}_{2}-\right), 3.21$ (t, $\left.J=6.5 \mathrm{~Hz}, 2 \mathrm{H},-\mathrm{CH}_{2}-\right), 2.91\left(\mathrm{t}, J=6.0 \mathrm{~Hz}, 4 \mathrm{H},-\mathrm{CH}_{2^{-}}\right), 1.94-1.85(\mathrm{~m}, 2 \mathrm{H}), 1.45(\mathrm{~m}, 2 \mathrm{H}), 1.32$ $(\mathrm{m}, 2 \mathrm{H}), 1.08-0.93(\mathrm{~m}, 4 \mathrm{H}), 0.90-0.76(\mathrm{~m}, 12 \mathrm{H}), 0.75-0.66(\mathrm{~m}, 4 \mathrm{H}), 0.64-0.58(\mathrm{~m}, 7 \mathrm{H})$, $0.54(\mathrm{~m}, 10 \mathrm{H})$. DEPTQ ${ }^{13} \mathrm{C} \mathrm{NMR}\left(125 \mathrm{MHz}, \mathrm{CDCl}_{3}, 300 \mathrm{~K}\right) \delta$ (ppm) 156.74, 156.24, 156.10, 
$155.97,155.94,155.92,155.80,155.15,154.99,150.07,149.65,149.31,149.14,149.08$, $149.06,139.95,139.74,138.19$, 137.95, 137.40, 136.97, 136.80, 136.73, 136.68, 135.42, 134.23 , 132.65, 132.05, 131.68, 131.46, 130.72, 130.19, 129.12, 128.24, 127.88, 127.86, $127.40,127.34,126.74,126.59,126.12,123.85,123.77,123.73,123.63,121.33,121.22$, $121.19,119.96,118.80,118.72,118.63,113.06,73.63,73.29,69.16,31.53,31.36,31.29,29.68$, 29.50, 29.17, 26.11, 25.36, 25.20, 22.57, 22.53, 22.42, 14.09, 13.92, 13.84. HR ESI-MS ( $\mathrm{m} / \mathrm{z})$ : calcd. for $\left[\mathrm{M}-\mathrm{BF}_{4}^{-}+2 \mathrm{H}\right]^{3+} 799.3774$ (found 799.3788), calcd. for $\left[\mathrm{M}-\mathrm{BF}_{4}{ }^{-}+3 \mathrm{H}\right]^{4+} 599.7850$ (found 599.7855).

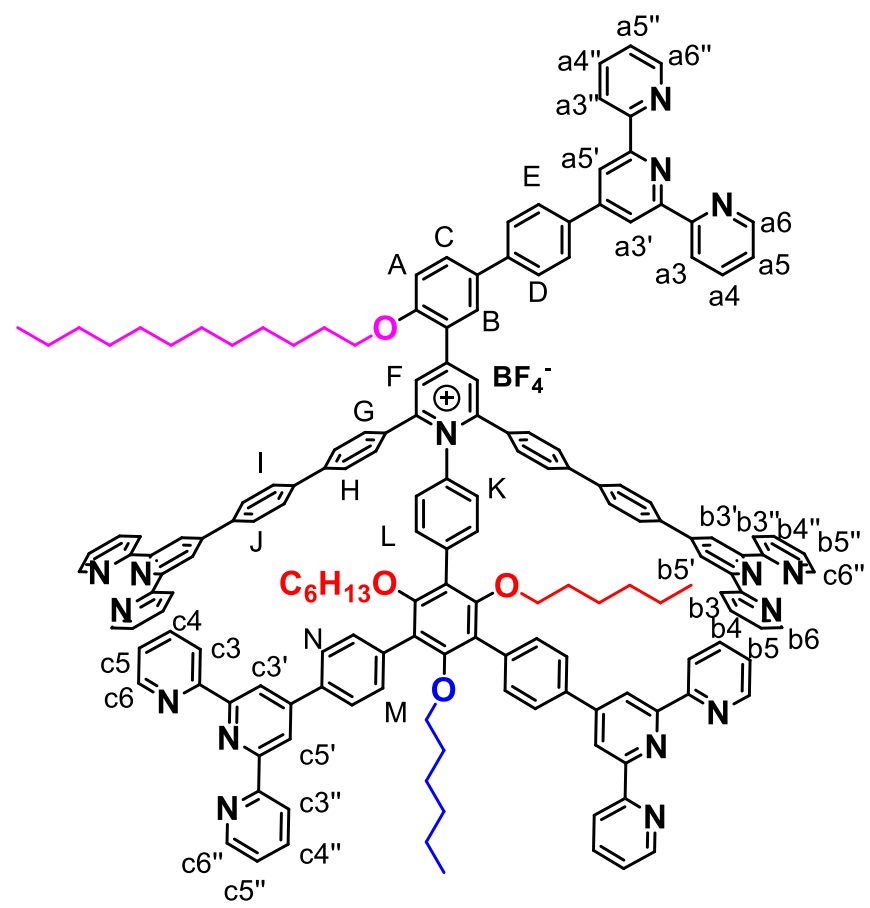

Ligand L2: L2 was prepared using the similar approach as L1. $300 \mathrm{mg}$ Compound 8 produced $196 \mathrm{mg}$ yellow powder (yield: 38\%). ${ }^{1} \mathrm{H}$ NMR (600 MHz, $\left.\mathrm{CDCl}_{3}, 300 \mathrm{~K}\right) \delta(\mathrm{ppm}) 8.78(\mathrm{~s}, 2 \mathrm{H}$, TPY- $\left.H^{\mathrm{a} 3^{\prime}, 5^{\prime}}\right), 8.75-8.73\left(\mathrm{~m}, 2 \mathrm{H}, \mathrm{TPY}-H^{\mathrm{a} 6,6^{\prime}}\right), 8.73$ (s, 4H, TPY- $\left.H^{\mathrm{b} 3^{\prime}, 5^{\prime}}\right), 8.71$ (s, 4H, TPY- $\left.H^{\mathrm{c} 3^{\prime}, 5^{\prime}}\right)$, 8.67 (d, $J=7.9$ Hz, 2H, TPY- $\left.H^{\mathrm{a} 3,3 ”}\right), 8.63$ (m, 16H, TPY- $H^{\mathrm{b}, \mathrm{c} 6,6 "}$; TPY- $\left.H^{\mathrm{b}, \mathrm{c} 3,3 "}\right), 8.41$ (s, 2H, Py$\left.H^{\mathrm{F}}\right), 8.20\left(\mathrm{~d}, J=2.2 \mathrm{~Hz}, 1 \mathrm{H}, \mathrm{Ph}-H^{\mathrm{B}}\right), 8.01\left(\mathrm{~d}, J=8.4 \mathrm{~Hz}, 2 \mathrm{H}, \mathrm{Ph}-H^{\mathrm{E}}\right), 7.97(\mathrm{~d}, J=8.7 \mathrm{~Hz}, 2 \mathrm{H}$, $\left.\mathrm{Ph}-H^{\mathrm{K}}\right), 7.94\left(\mathrm{~d}, J=8.4 \mathrm{~Hz}, 4 \mathrm{H}, \mathrm{Ph}-H^{\mathrm{G}}\right), 7.92-7.80\left(\mathrm{~m}, 21 \mathrm{H}, \mathrm{Ph}-H^{\mathrm{J}, \mathrm{N}}, \mathrm{TPY}-H^{\mathrm{a}-\mathrm{c} 4,4}, \mathrm{Ph}-H^{\mathrm{C}, \mathrm{D}}\right)$, $7.65\left(\mathrm{~m}, 8 \mathrm{H}, \mathrm{Ph}-H^{\mathrm{I}, \mathrm{H}}\right), 7.57\left(\mathrm{~d}, J=8.8 \mathrm{~Hz}, 2 \mathrm{H}, \mathrm{Ph}-H^{\mathrm{L}}\right), 7.55\left(\mathrm{~d}, J=8.3 \mathrm{~Hz}, 4 \mathrm{H}, \mathrm{Ph}-H^{\mathrm{M}}\right), 7.35$ (ddd, $J=7.5,4.7,1.1 \mathrm{~Hz}, 2 \mathrm{H}$, TPY- $H^{\mathrm{a}, 5}$ ") $) 7.30-7.26$ (m, 8H, TPY- $\left.H^{\mathrm{b}, \mathrm{c} 5,5 ”}\right), 7.19$ (d, $J=8.7$ $\left.\mathrm{Hz}, 1 \mathrm{H}, \mathrm{Ph}-H^{\mathrm{A}}\right), 4.20\left(\mathrm{t}, J=6.3 \mathrm{~Hz}, 2 \mathrm{H},-\mathrm{CH}_{2}-\right), 3.21\left(\mathrm{t}, J=6.5 \mathrm{~Hz}, 2 \mathrm{H},-\mathrm{CH}_{2}-\right), 2.91(\mathrm{t}, J=$ $\left.6.0 \mathrm{~Hz}, 4 \mathrm{H},-\mathrm{CH}_{2}-\right), 1.95-1.86(\mathrm{~m}, 2 \mathrm{H}), 1.45(\mathrm{~m}, 2 \mathrm{H}), 1.32(\mathrm{~m}, 3 \mathrm{H}), 1.07-0.94(\mathrm{~m}, 5 \mathrm{H}), 0.91$ 
$-0.75(\mathrm{~m}, 13 \mathrm{H}), 0.75-0.65(\mathrm{~m}, 5 \mathrm{H}), 0.65-0.59(\mathrm{~m}, 7 \mathrm{H}), 0.59-0.47(\mathrm{~m}, 11 \mathrm{H})$. DEPTQ ${ }^{13} \mathrm{C}$ NMR (125 MHz, $\left.\mathrm{CDCl}_{3}, 300 \mathrm{~K}\right) \delta(\mathrm{ppm}) 156.77,156.25,156.11,156.00,155.99,155.93$, $155.80,155.15,154.86,150.08,149.67,149.31$, 149.15, 149.08, 149.07, 141.65, 139.95, $139.75,138.19,138.01,137.43,136.97,136.81,136.72,136.68,135.44,134.25,132.68$, $132.07,131.65,131.46,130.77,130.17,129.10,128.30,127.88,127.41,127.35,126.73$, $126.59,126.12$, 123.84, 123.82, 123.77, 123.72, 123.63, 121.34, 121.22, 121.19, 118.81, 118.74, 118.64, 113.07, 73.63, 73.29, 69.17, 31.88, 31.40, 31.36, 31.29, 29.71, 29.69, 29.67, $29.60,29.50,29.41,29.35,29.22,26.43,25.36,25.21,22.63,22.57,22.42,14.05,13.92,13.84$. HR ESI-MS $(m / z)$ : calcd. for $\left[\mathrm{M}-\mathrm{BF}_{4}{ }^{-}+2 \mathrm{H}\right]^{3+} 827.4087$ (found 827.4105), calcd. for $\left[\mathrm{M}-\mathrm{BF}_{4}{ }^{-}+3 \mathrm{H}\right]^{4+} 620.8085$ (found 620.8069). 


\section{Synthesis of the Complexes HP1 and HP2}

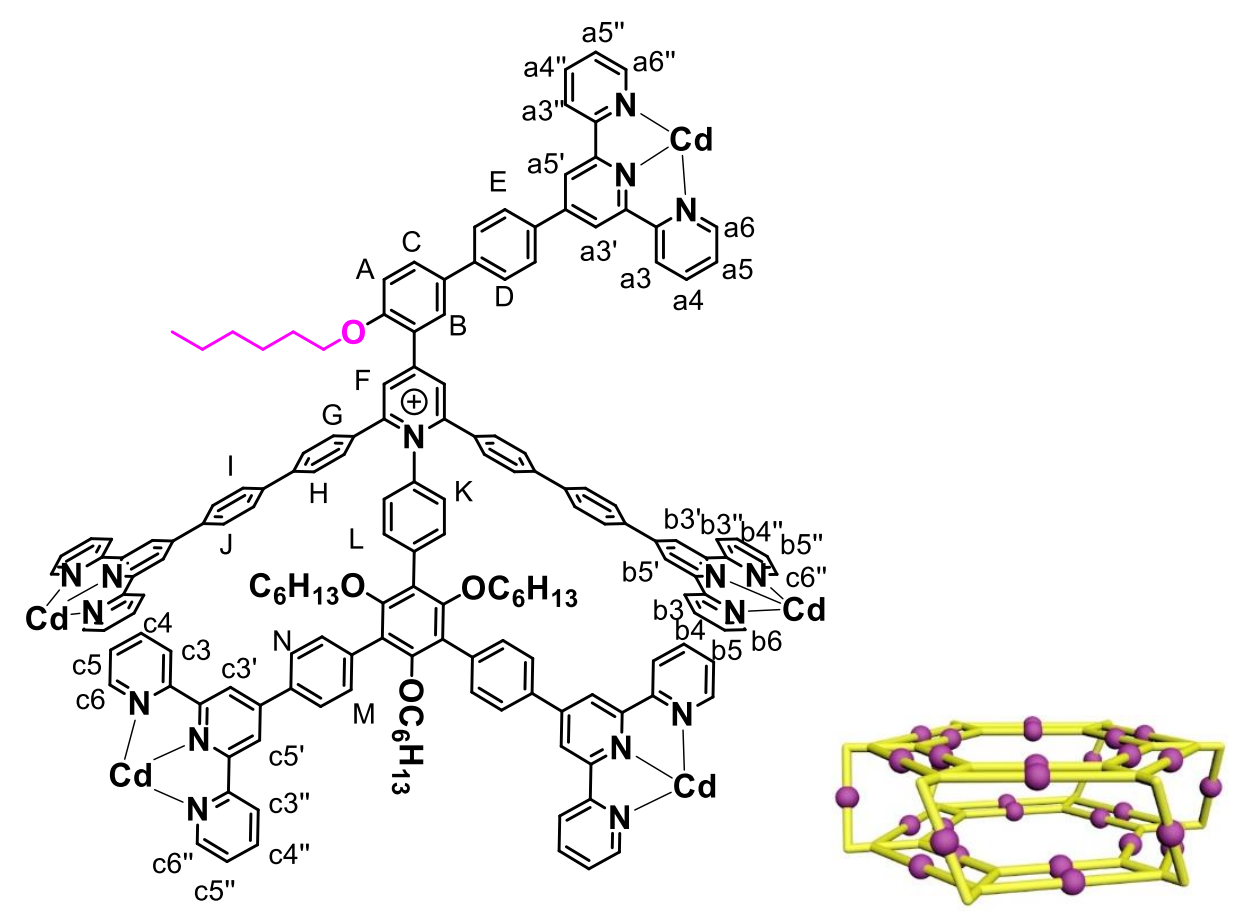

HP1: To a solution of ligand $\mathbf{L 1}(10.0 \mathrm{mg}, 4.03 \mu \mathrm{mol})$ in DMSO $(0.5 \mathrm{~mL})$, a solution of $\mathrm{Cd}\left(\mathrm{NO}_{3}\right)_{2} \cdot 4 \mathrm{H}_{2} \mathrm{O}(3.1 \mathrm{mg}, 10 \mu \mathrm{mol})$ in $\mathrm{DMSO}(0.5 \mathrm{~mL})$ was added, and then the mixture was kept at $80{ }^{\circ} \mathrm{C}$ for $12 \mathrm{~h}$. After cooling to room temperature, the assembly solution was added to $10 \mathrm{~mL} \mathrm{CH}_{3} \mathrm{OH}$ solution of $\mathrm{NH}_{4} \mathrm{PF}_{6}(80 \mathrm{mg})$, and yellow precipitate was formed. The precipitate was collected by centrifugation and washed by fresh $\mathrm{CH}_{3} \mathrm{OH}$ and water repeatedly. $10.5 \mathrm{mg}$ yellow solid was obtained (yield 74\%). ${ }^{1} \mathrm{H}$ NMR (800 MHz, $d_{6}$-DMSO, $\left.300 \mathrm{~K}\right) \delta$ (ppm) 8.96, 9.02 (s, 2H, TPY- $H^{\mathrm{a}^{\prime}}$, TPY- $\left.H^{\mathrm{a} 5^{\prime}}\right), 8.81\left(\mathrm{~s}, 4 \mathrm{H}, \mathrm{TPY}-H^{\mathrm{b} 3^{\prime}, 5^{\prime}}\right), 8.77\left(\mathrm{~s}, 4 \mathrm{H}, \mathrm{TPY}-H^{\mathrm{c}^{\prime}, 5^{\prime}}\right), 8.67$ (m, 9H, TPY- $H^{\mathrm{a} 3}$, TPY- $H^{\mathrm{b} 3,3 ”}$, Ph- $\left.H^{\mathrm{N}}\right), 8.62\left(\mathrm{~m}, 5 \mathrm{H}, \mathrm{TPY}-H^{\mathrm{a} 3 ”}, \mathrm{Ph}-H^{\mathrm{G}}\right), 8.58\left(\mathrm{~m}, 6 \mathrm{H}, \mathrm{TPY}-H^{\mathrm{c} 3,3 ”}\right.$, Ph- $\left.H^{\mathrm{E}}\right), 8.39\left(\mathrm{~m}, 4 \mathrm{H}, \mathrm{Ph}-H^{\mathrm{J}}\right), 8.28\left(\mathrm{~s}, 2 \mathrm{H}, \mathrm{Py}-H^{\mathrm{F}}\right), 8.07$ (m, 6H, TPY- $\left.H^{\mathrm{a} 4,4}, \mathrm{Ph}-H^{\mathrm{I}}\right), 8.00$ (br, 13H, TPY- $\left.H^{\mathrm{b}-\mathrm{c} 4,4^{\prime}}, \mathrm{Ph}-H^{\mathrm{B}, \mathrm{H}}\right), 7.92\left(\mathrm{~m}, 2 \mathrm{H}, \mathrm{TPY}-H^{\mathrm{a} 6,6 ”}\right), 7.89\left(\mathrm{~m}, 10 \mathrm{H}, \mathrm{TPY}-H^{\mathrm{b}-\mathrm{c} 6,6 ”}, \mathrm{Ph}-H^{\mathrm{K}}\right)$, $7.74\left(\mathrm{~m}, 5 \mathrm{H}, \mathrm{TPY}-H^{\mathrm{b} 5,5 ”}, \mathrm{Ph}-H^{\mathrm{C}}\right), 7.54\left(\mathrm{~m}, 6 \mathrm{H}, \mathrm{TPY}-H^{\mathrm{a} 5,5^{\prime}}, \mathrm{Ph}-H^{\mathrm{M}}\right), 7.50\left(\mathrm{~m}, 3 \mathrm{H}, \mathrm{Ph}-H^{\mathrm{A}, \mathrm{L}}\right)$, $7.43\left(\mathrm{~m}, 6 \mathrm{H}, \mathrm{TPY}-H^{\mathrm{c} 5,5^{\prime \prime}}, \mathrm{Ph}-H^{\mathrm{D}}\right), 4.27\left(\mathrm{~m}, 2 \mathrm{H},-\mathrm{CH}_{2^{-}}\right), 2.80\left(\mathrm{~m}, 4 \mathrm{H},-\mathrm{CH}_{2^{-}}\right), 1.77(\mathrm{~m}, 2 \mathrm{H}), 1.35$ (m, 3H), $1.16(\mathrm{~m}, 3 \mathrm{H}), 1.07(\mathrm{~m}, 3 \mathrm{H}), 0.96(\mathrm{~m}, 3 \mathrm{H}), 0.87(\mathrm{~m}, 4 \mathrm{H}), 0.72(\mathrm{~m}, 10 \mathrm{H}), 0.55(\mathrm{~m}, 4 \mathrm{H})$, $0.47(\mathrm{~m}, 11 \mathrm{H}), 0.20(\mathrm{~m}, 15 \mathrm{H}), 0.11(\mathrm{~m}, 3 \mathrm{H})$. DEPTQ ${ }^{13} \mathrm{C}$ NMR spectrum was shown in Figure S36. The peaks were not listed due to the low intensity of the signals in the aromic region. ESIMS $(m / z): 2516.8\left[\mathrm{M}^{-16 \mathrm{PF}_{6}}{ }^{-}\right]^{16+}$ (calcd. $\left.\mathrm{m} / z: 2516.7\right), 2360.2\left[\mathrm{M}-17 \mathrm{PF}_{6}{ }^{-}\right]^{17+}$ (calcd. $\mathrm{m} / z$ : 


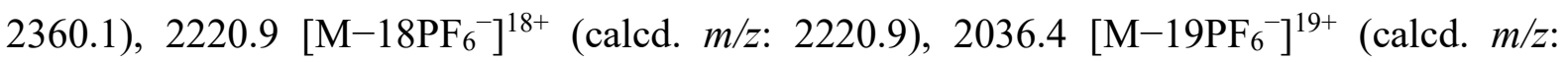

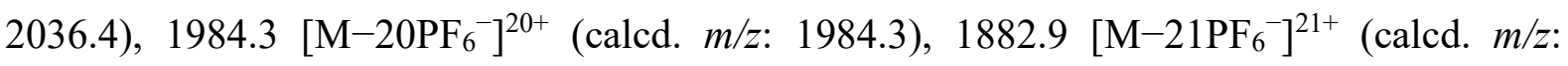

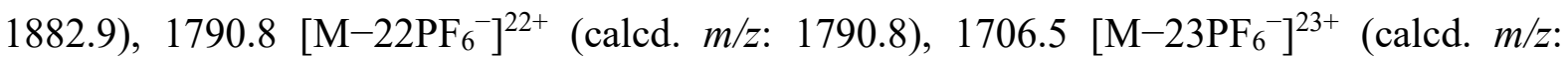
1706.6), $1629.3\left[\mathrm{M}^{\left.-24 \mathrm{PF}_{6}\right]^{-24+}}\right.$ (calcd. $\left.m / z: 1629.4\right), 1558.4\left[\mathrm{M}-25 \mathrm{PF}_{6}{ }^{-25+}\right.$ (calcd. $m / z$ : 1558.4), $1493.0\left[\mathrm{M}-26 \mathrm{PF}_{6}\right]^{26+}$ (calcd. $m / z: 1493.0$ ).
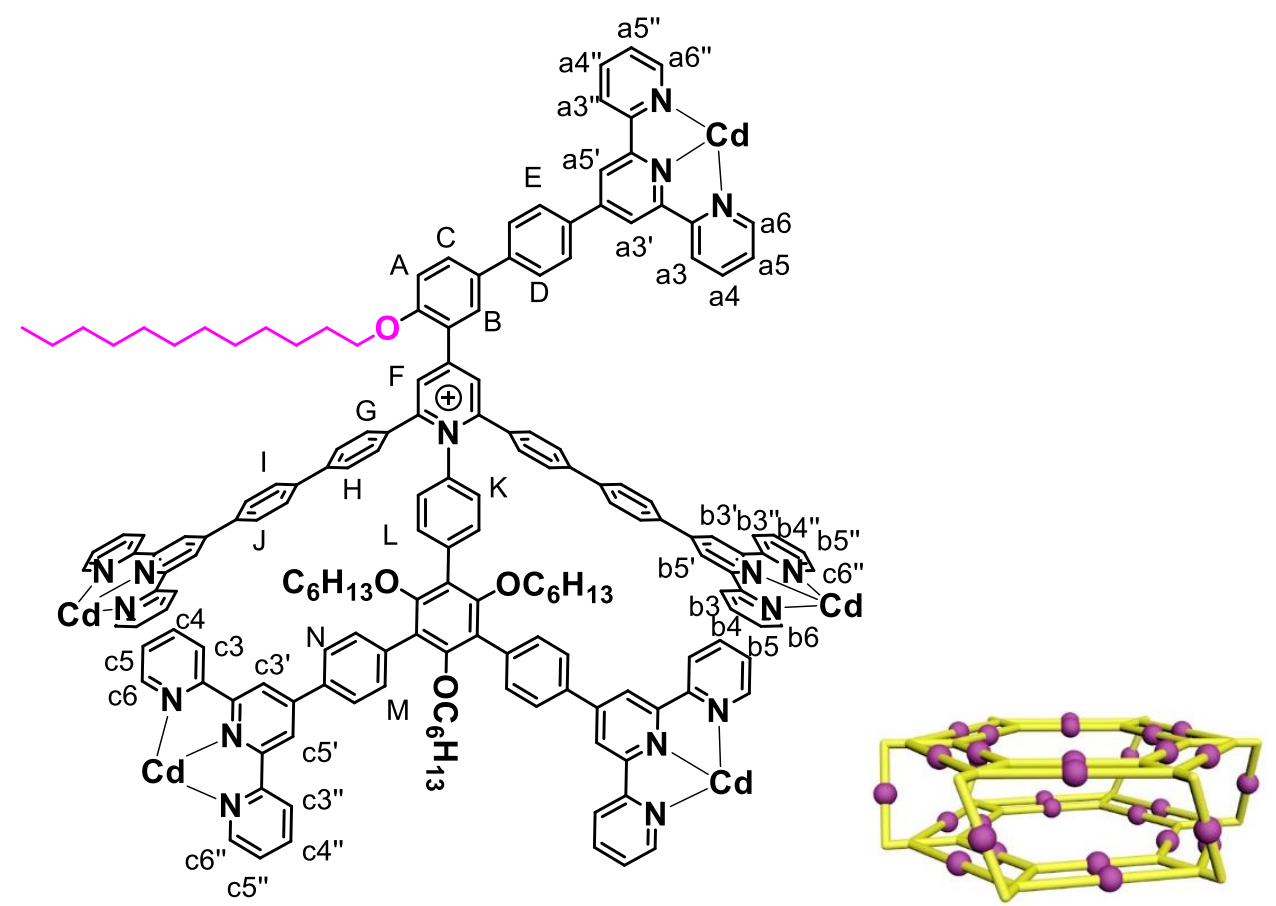

HP2: To a solution of ligand $\mathbf{L 2}(10.3 \mathrm{mg}, 4.00 \mu \mathrm{mol})$ in DMSO $(0.5 \mathrm{~mL})$, a solution of $\mathrm{Cd}\left(\mathrm{NO}_{3}\right)_{2} \cdot{ }_{4} \mathrm{H}_{2} \mathrm{O}(3.1 \mathrm{mg}, 10 \mu \mathrm{mol})$ in DMSO $(0.5 \mathrm{~mL})$ was added, and then the mixture was kept at $80^{\circ} \mathrm{C}$ for $12 \mathrm{~h}$. After cooling to room temperature, the assembly solution was added to $10 \mathrm{~mL} \mathrm{CH}_{3} \mathrm{OH}$ solution of $\mathrm{NH}_{4} \mathrm{PF}_{6}(80 \mathrm{mg})$, and yellow precipitate was formed. The precipitate was collected by centrifugation and washed by fresh $\mathrm{CH}_{3} \mathrm{OH}$ and water repeatedly. $11.5 \mathrm{mg}$ yellow solid was obtained (yield 79\%). ${ }^{1} \mathrm{H}$ NMR (800 MHz, $d_{6}$-DMSO, $\left.300 \mathrm{~K}\right) \delta(\mathrm{ppm}) 9.03$, 8.97 (s, 2H, TPY- $H^{\mathrm{a} 3^{\prime}}$, TPY- $\left.H^{\mathrm{a} 5^{\prime}}\right), 8.81$ (s, 4H, TPY- $\left.H^{\mathrm{b} 3^{\prime}, 5^{\prime}}\right), 8.77\left(\mathrm{~s}, 4 \mathrm{H}, \mathrm{TPY}-H^{\mathrm{c}^{\prime}, 5^{\prime}}\right), 8.68$ (m, 9H, TPY- $H^{\mathrm{a} 3}$, TPY- $H^{\mathrm{b} 3,3 "}$, Ph- $\left.H^{\mathrm{N}}\right), 8.62\left(\mathrm{~m}, 5 \mathrm{H}, \mathrm{TPY}-H^{\mathrm{a} 3 "}, \mathrm{Ph}-H^{\mathrm{G}}\right), 8.58\left(\mathrm{~m}, 6 \mathrm{H}\right.$, TPY- $H^{\mathrm{c} 3,3 "}$, Ph- $\left.H^{\mathrm{E}}\right), 8.40\left(\mathrm{~m}, 4 \mathrm{H}, \mathrm{Ph}-H^{\mathrm{J}}\right), 8.29\left(\mathrm{br}, 2 \mathrm{H}, \mathrm{Ph}-H^{\mathrm{F}}\right), 8.06\left(\mathrm{~m}, 6 \mathrm{H}, \mathrm{TPY}-H^{\mathrm{a} 4,4}\right.$ ”, Ph- $\left.H^{\mathrm{I}}\right), 7.98(\mathrm{~m}$, 13H, TPY- $\left.H^{\mathrm{b}-\mathrm{c} 4,4 "}, \mathrm{Ph}-H^{\mathrm{B}, \mathrm{H}}\right), 7.93\left(\mathrm{~m}, 2 \mathrm{H}, \mathrm{TPY}-H^{\mathrm{a} 6,6 ”}\right), 7.89\left(\mathrm{~m}, 10 \mathrm{H}, \mathrm{TPY}-H^{\mathrm{b}-\mathrm{c} 6,6 ”}, \mathrm{Ph}-H^{\mathrm{K}}\right)$, $7.74\left(\mathrm{~m}, 5 \mathrm{H}, \mathrm{TPY}-H^{\mathrm{b} 5,5^{\prime}}, \mathrm{Ph}-H^{\mathrm{C}}\right), 7.54\left(\mathrm{~m}, 6 \mathrm{H}, \mathrm{TPY}-H^{\mathrm{a} 5,5^{\prime}}, \mathrm{Ph}-H^{\mathrm{M}}\right), 7.50\left(\mathrm{~m}, 3 \mathrm{H}, \mathrm{Ph}-H^{\mathrm{A}, \mathrm{L}}\right)$, $7.43\left(\mathrm{~m}, 6 \mathrm{H}, \mathrm{TPY}-H^{\mathrm{c} 5,5^{\prime}}, \mathrm{Ph}-H^{\mathrm{D}}\right), 4.26\left(\mathrm{~m}, 2 \mathrm{H},-\mathrm{CH}_{2^{-}}\right), 2.80\left(\mathrm{~m}, 4 \mathrm{H},-\mathrm{CH}_{2^{-}}\right), 1.76(\mathrm{~m}, 3 \mathrm{H}), 1.32$ 
(m, 3H), $1.23(\mathrm{~m}, 4 \mathrm{H}), 1.17(\mathrm{~m}, 4 \mathrm{H}), 1.02(\mathrm{~m}, 25 \mathrm{H}), 0.88(\mathrm{~m}, 4 \mathrm{H}), 0.72(\mathrm{~m}, 6 \mathrm{H}), 0.57(\mathrm{~m}, 8 \mathrm{H})$, $0.47(\mathrm{~m}, 12 \mathrm{H}), 0.20(\mathrm{~m}, 18 \mathrm{H})$. DEPTQ ${ }^{13} \mathrm{C}$ NMR spectrum was shown in Figure S42. The peaks were not listed due to the low intensity of the signals in the aromic region. ESI-MS $(\mathrm{m} / \mathrm{z})$ : $2579.8\left[\mathrm{M}-16 \mathrm{PF}_{6}{ }^{-}\right]^{16+}$ (calcd. $\left.m / z: 2579.7\right), 2419.7\left[\mathrm{M}-17 \mathrm{PF}_{6}{ }^{-}\right]^{17+}$ (calcd. $\left.m / z: 2419.5\right), 2277.1$ $\left[\mathrm{M}-18 \mathrm{PF}_{6}{ }^{-}\right]^{18+}$ (calcd. $\left.m / z: 2277.0\right), 2149.7\left[\mathrm{M}-19 \mathrm{PF}_{6}{ }^{-}\right]^{19+}$ (calcd. $\left.\mathrm{m} / z: 2049.6\right), 2034.9$

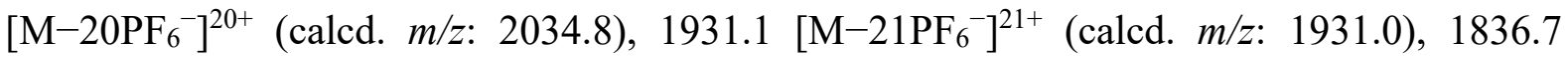
$\left[\mathrm{M}-22 \mathrm{PF}_{6}{ }^{-}\right]^{22+}$ (calcd. $\left.m / z: 1836.6\right), 1750.5\left[\mathrm{M}-23 \mathrm{PF}_{6}{ }^{-23+}\right.$ (calcd. $\left.\mathrm{m} / z: 1750.5\right), 1671.5$ $\left[\mathrm{M}-24 \mathrm{PF}_{6}{ }^{-}\right]^{24+}$ (calcd. $\left.\mathrm{m} / \mathrm{z}: 1671.5\right), 1598.7\left[\mathrm{M}-25 \mathrm{PF}_{6}{ }^{-25+}\right.$ (calcd. $\left.\mathrm{m} / z: 1598.8\right), 1531.9$ $\left[\mathrm{M}-26 \mathrm{PF}_{6}{ }^{-}\right]^{26+}$ (calcd. $m / z$ : 1531.8). 


\section{4. ${ }^{1} \mathrm{H}$ NMR, DEPTQ ${ }^{13} \mathrm{C}$ NMR, 2D COSY NMR, 2D NOESY NMR, and ESI-MS spectra}

of Compounds 1-8 and Ligands L1-L2

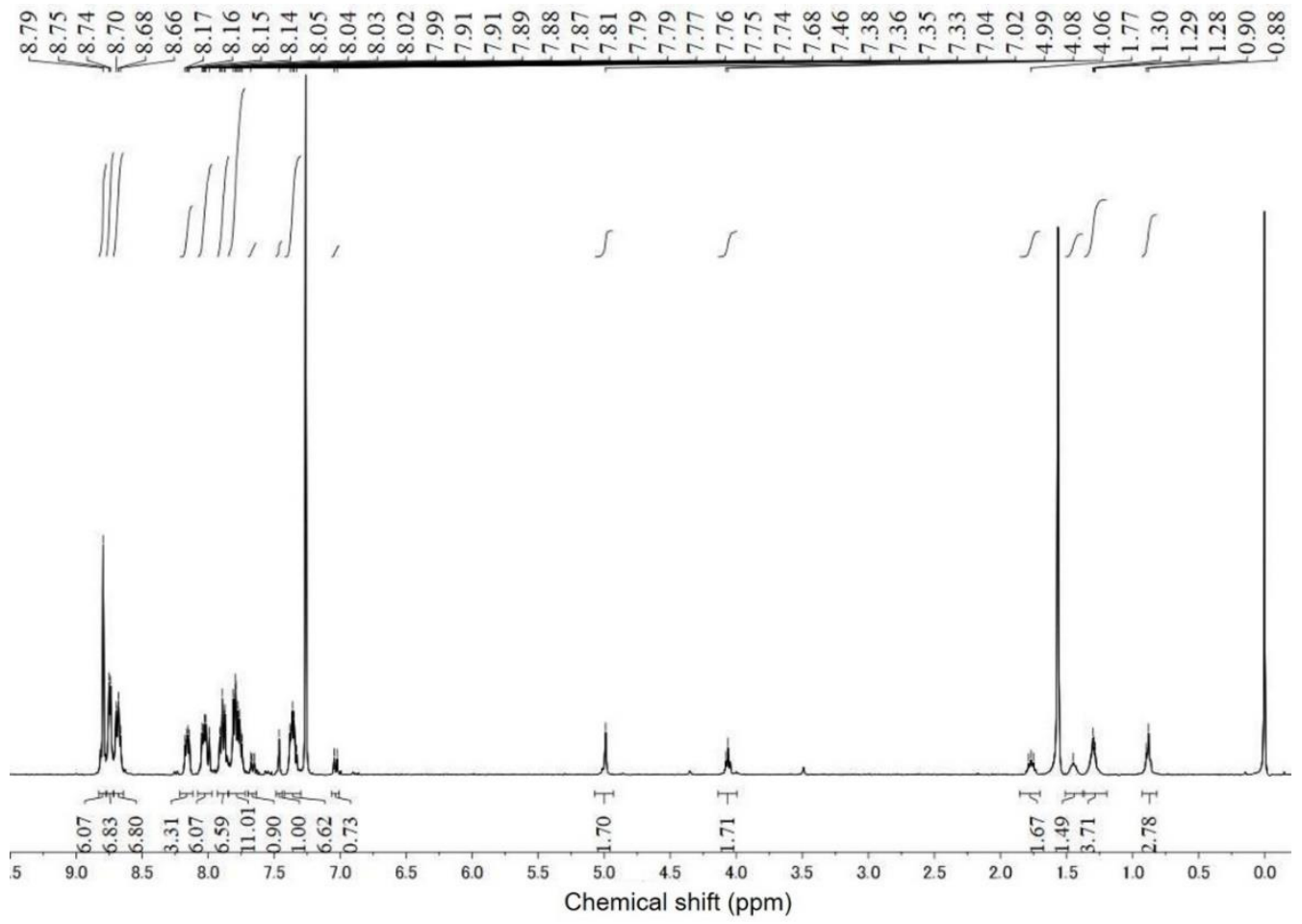

Figure S1: ${ }^{1} \mathrm{H}$ NMR $\left(400 \mathrm{MHz}, \mathrm{CDCl}_{3}, 300 \mathrm{~K}\right)$ spectrum of Compound 5.

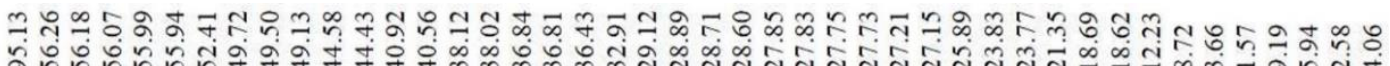

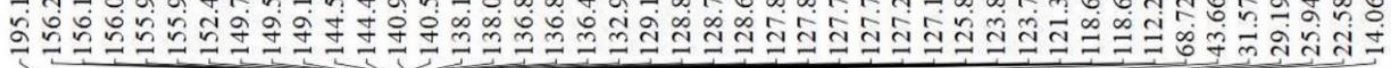

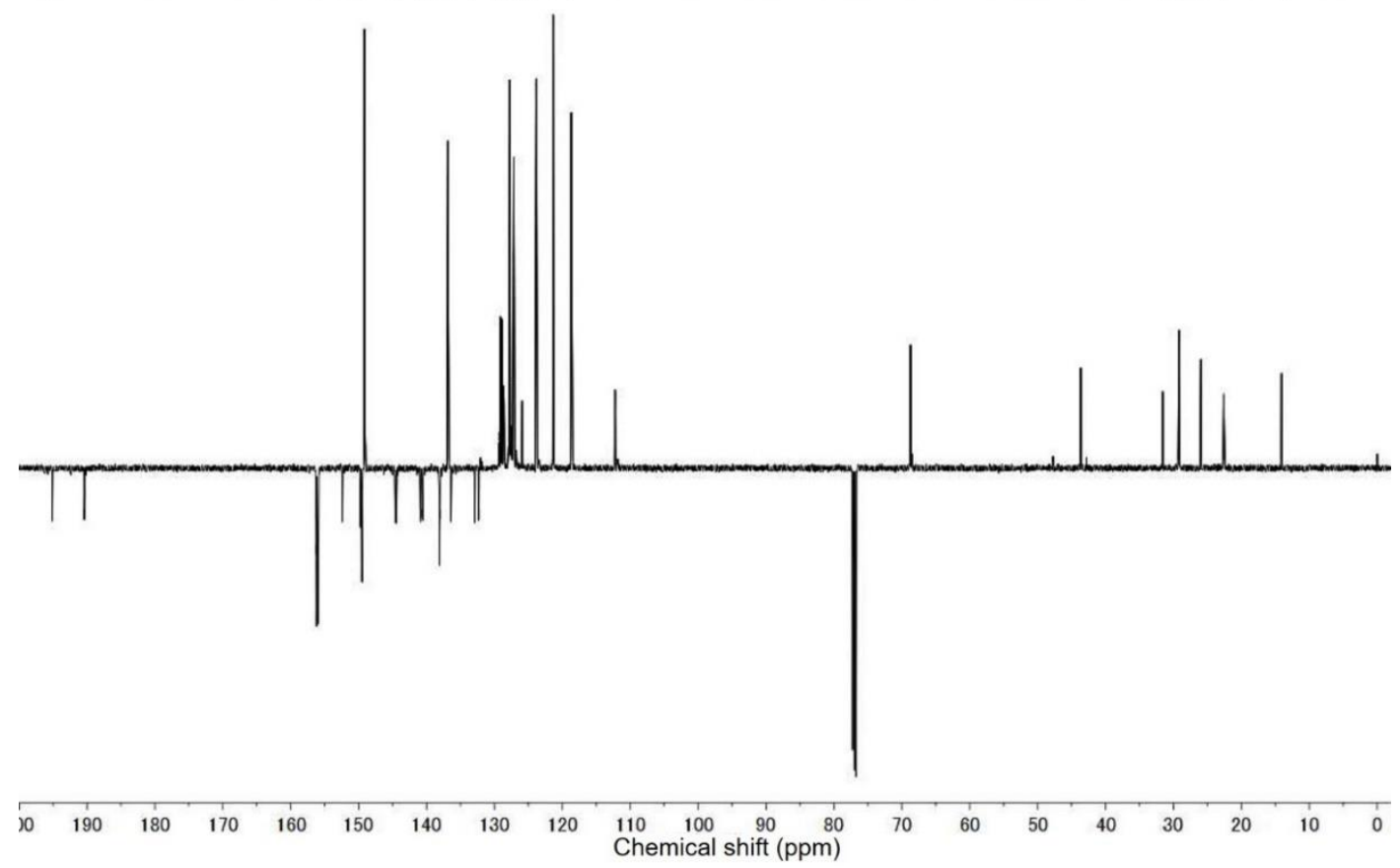

Figure S2: DEPTQ ${ }^{13} \mathrm{C}$ NMR $\left(125 \mathrm{MHz}, \mathrm{CDCl}_{3}, 300 \mathrm{~K}\right)$ spectrum of Compound 5. 


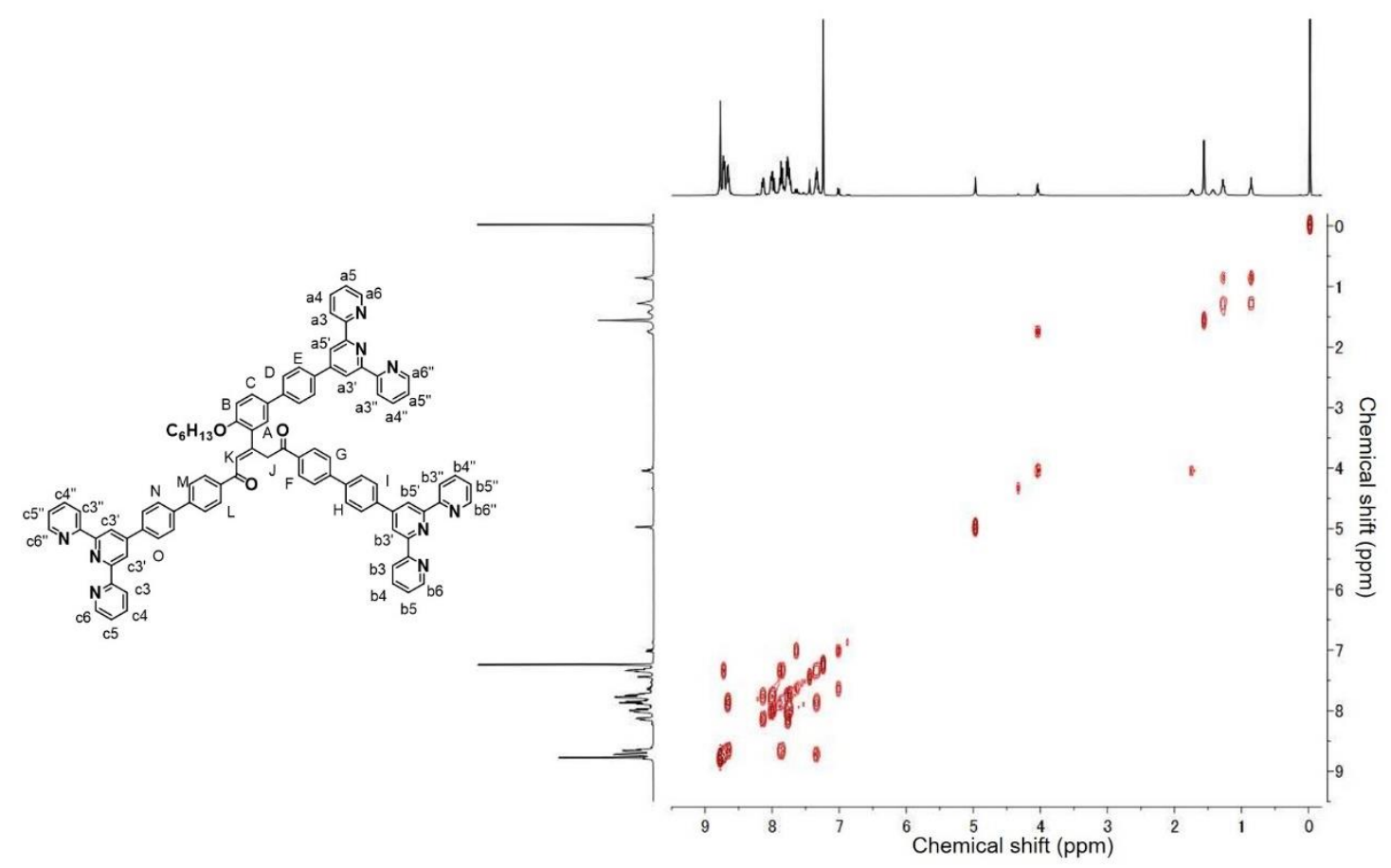

Figure S3: 2D COSY NMR (400 MHz, $\mathrm{CDCl}_{3}, 300 \mathrm{~K}$ ) spectrum of Compound 5.

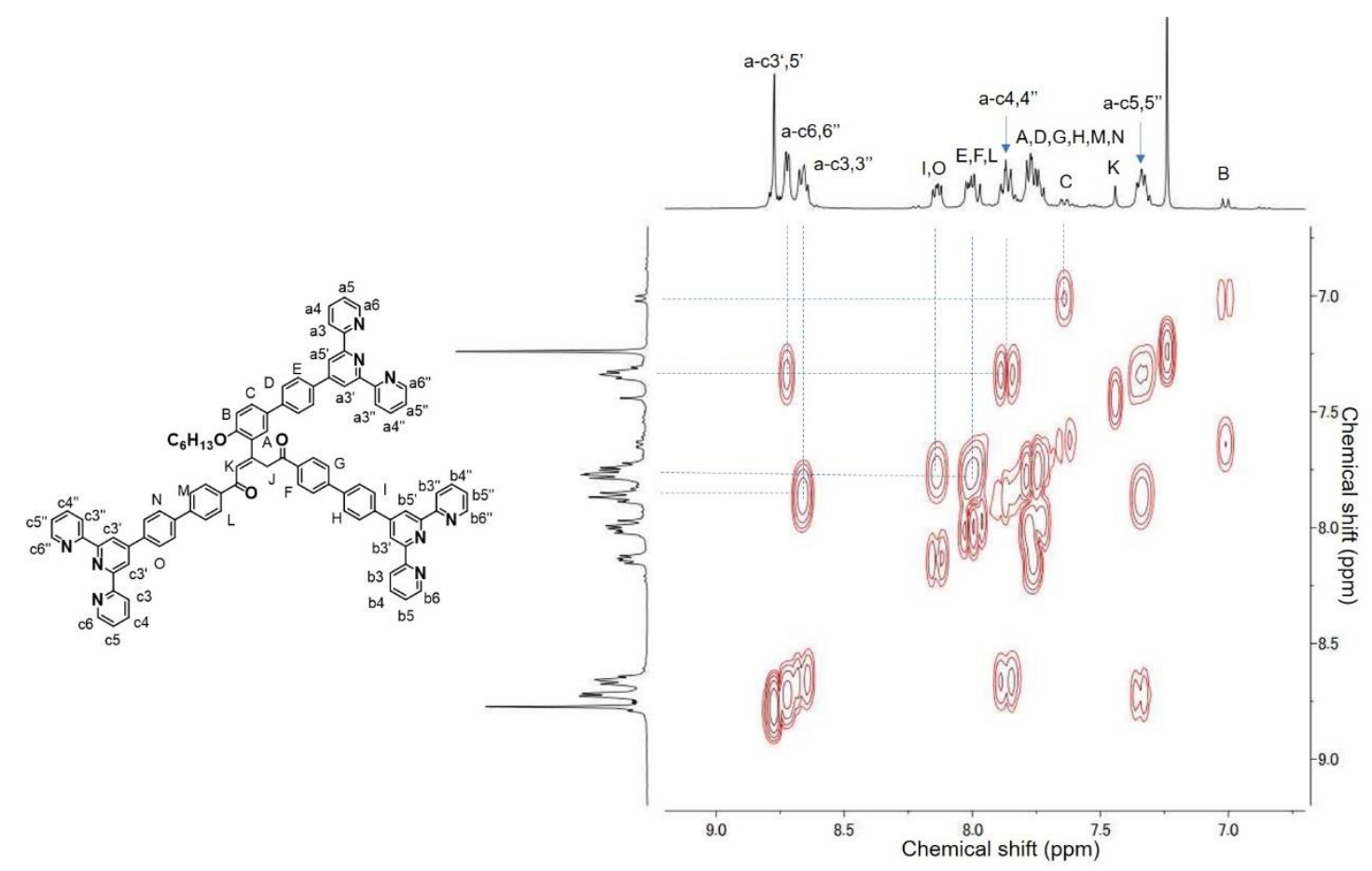

Figure S4: 2D COSY NMR (400 MHz, $\mathrm{CDCl}_{3}, 300 \mathrm{~K}$ ) spectrum of Compound 5 (aromatic region). 


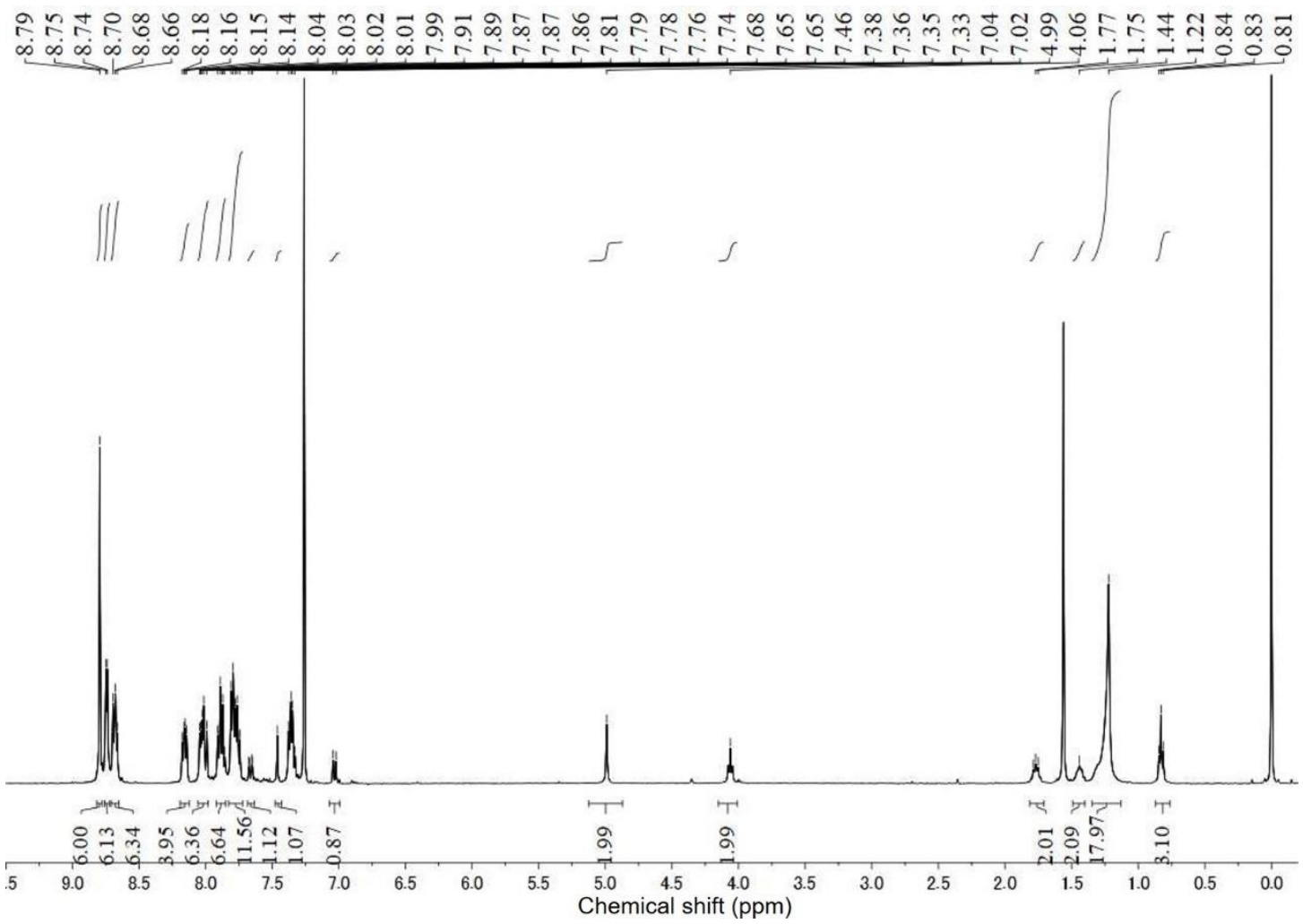

Figure S5: ${ }^{1} \mathrm{H}$ NMR $\left(400 \mathrm{MHz}, \mathrm{CDCl}_{3}, 300 \mathrm{~K}\right)$ spectrum of Compound 6.

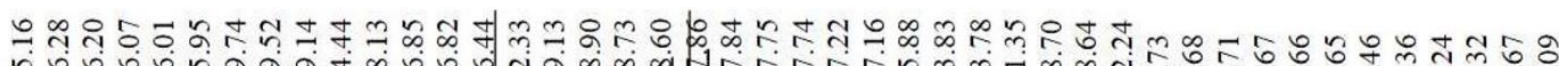

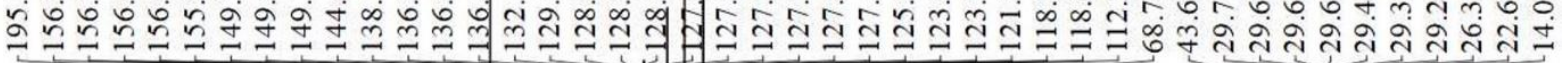

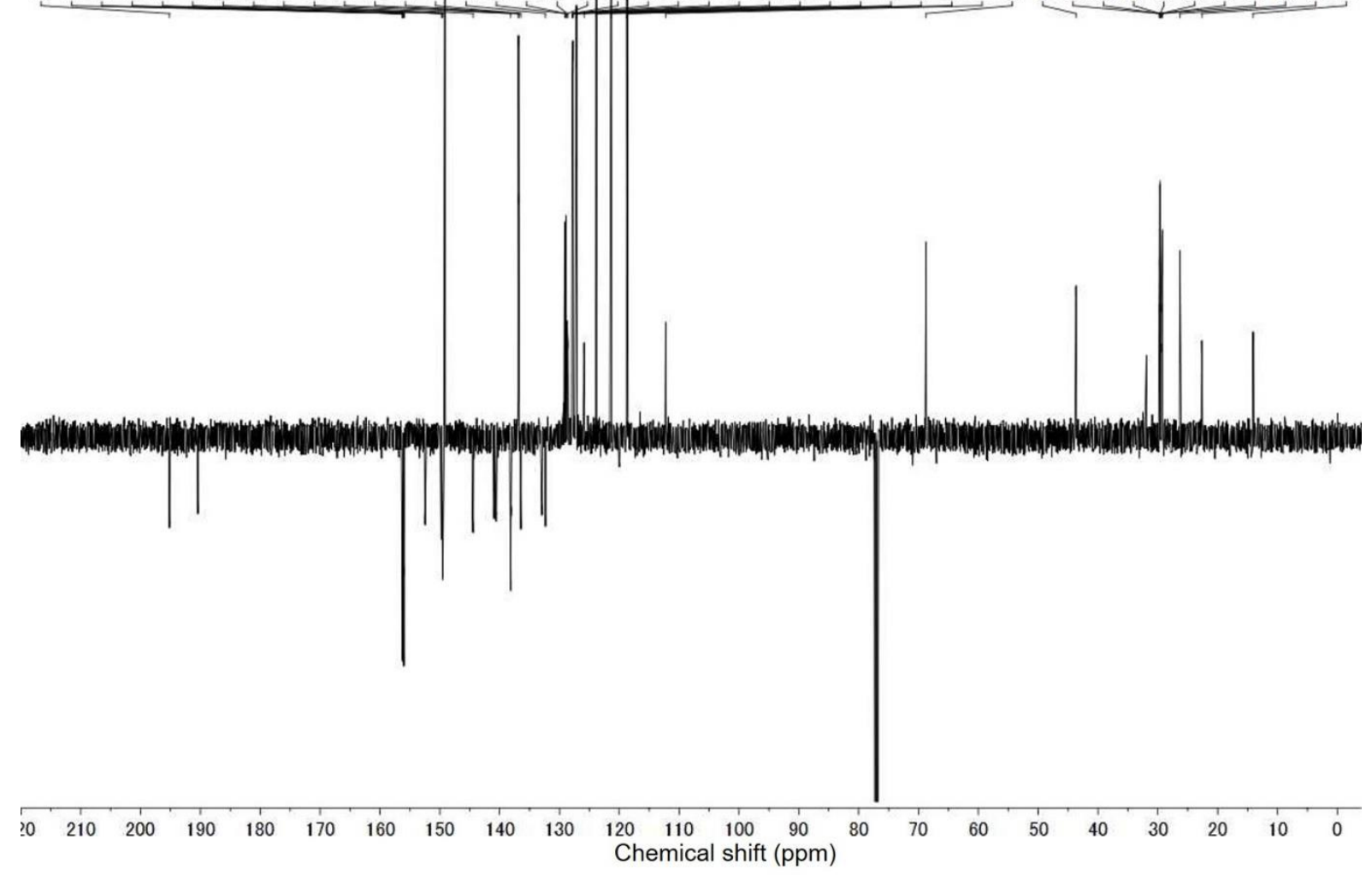

Figure S6: DEPTQ ${ }^{13} \mathrm{C}$ NMR $\left(125 \mathrm{MHz}, \mathrm{CDCl}_{3}, 300 \mathrm{~K}\right)$ spectrum of Compound 6. 


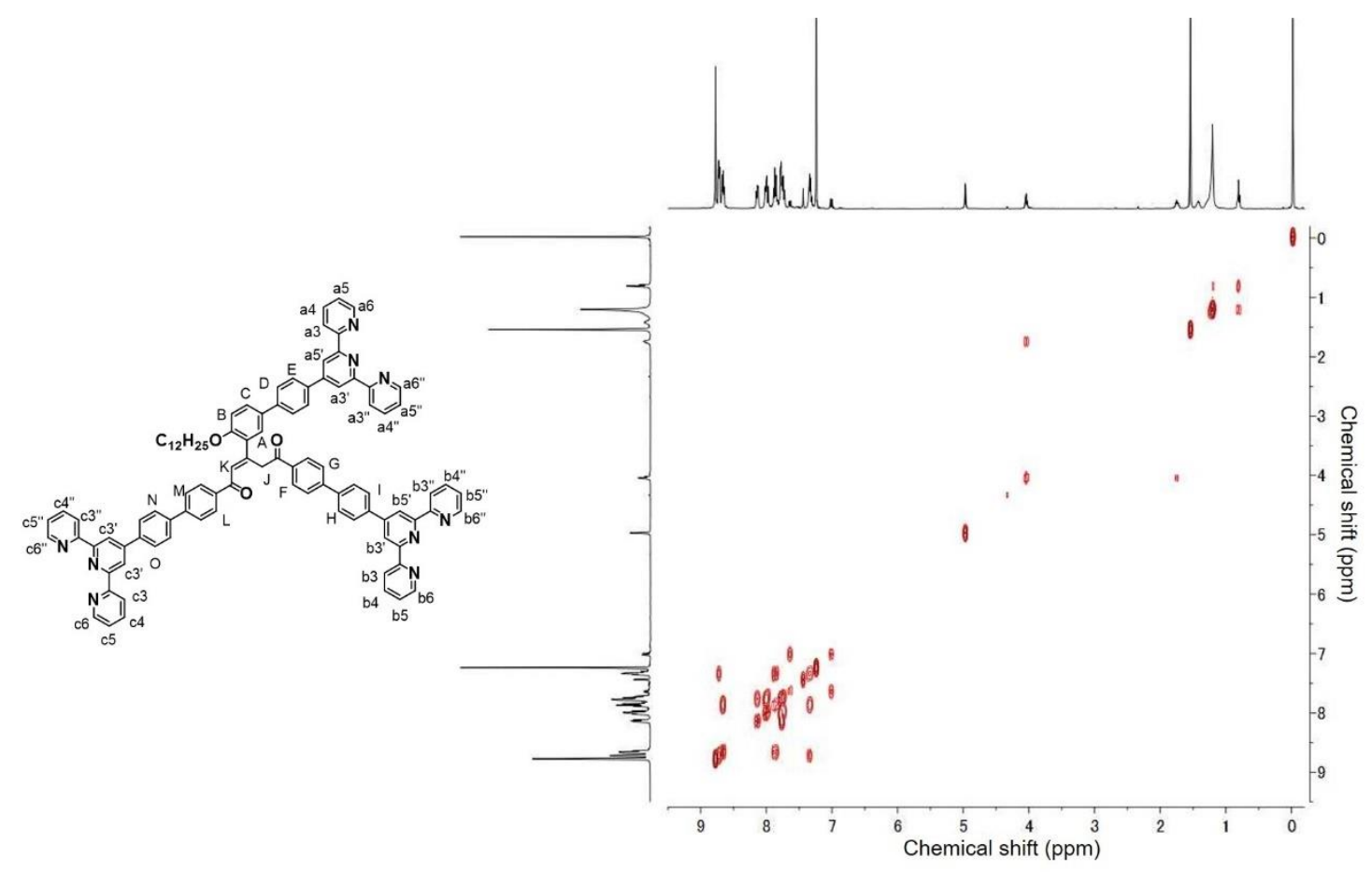

Figure S7: 2D COSY NMR (400 MHz, $\mathrm{CDCl}_{3}, 300 \mathrm{~K}$ ) spectrum of Compound 6.

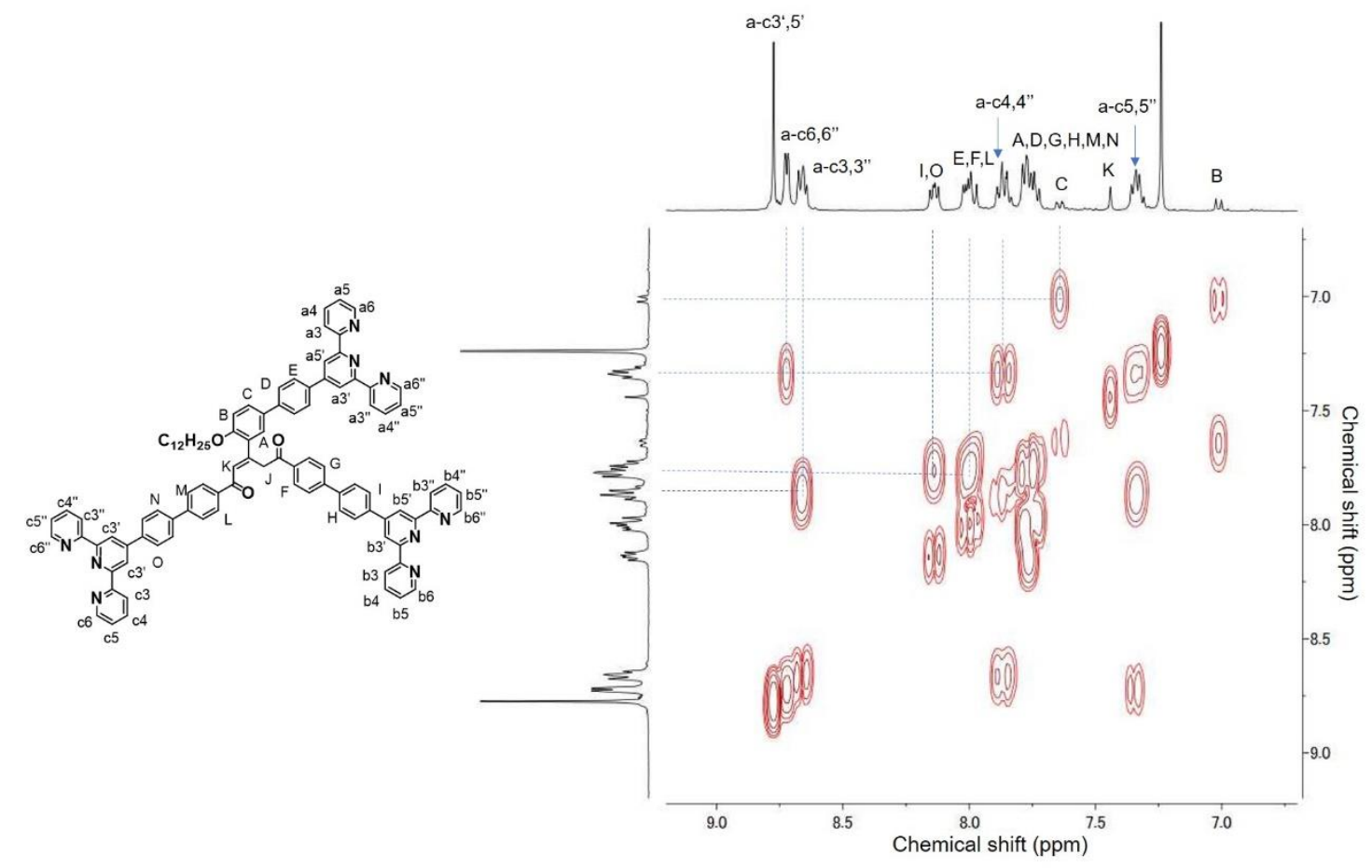

Figure S8: 2D COSY NMR (400 MHz, $\mathrm{CDCl}_{3}, 300 \mathrm{~K}$ ) spectrum of Compound 6 (aromatic region). 


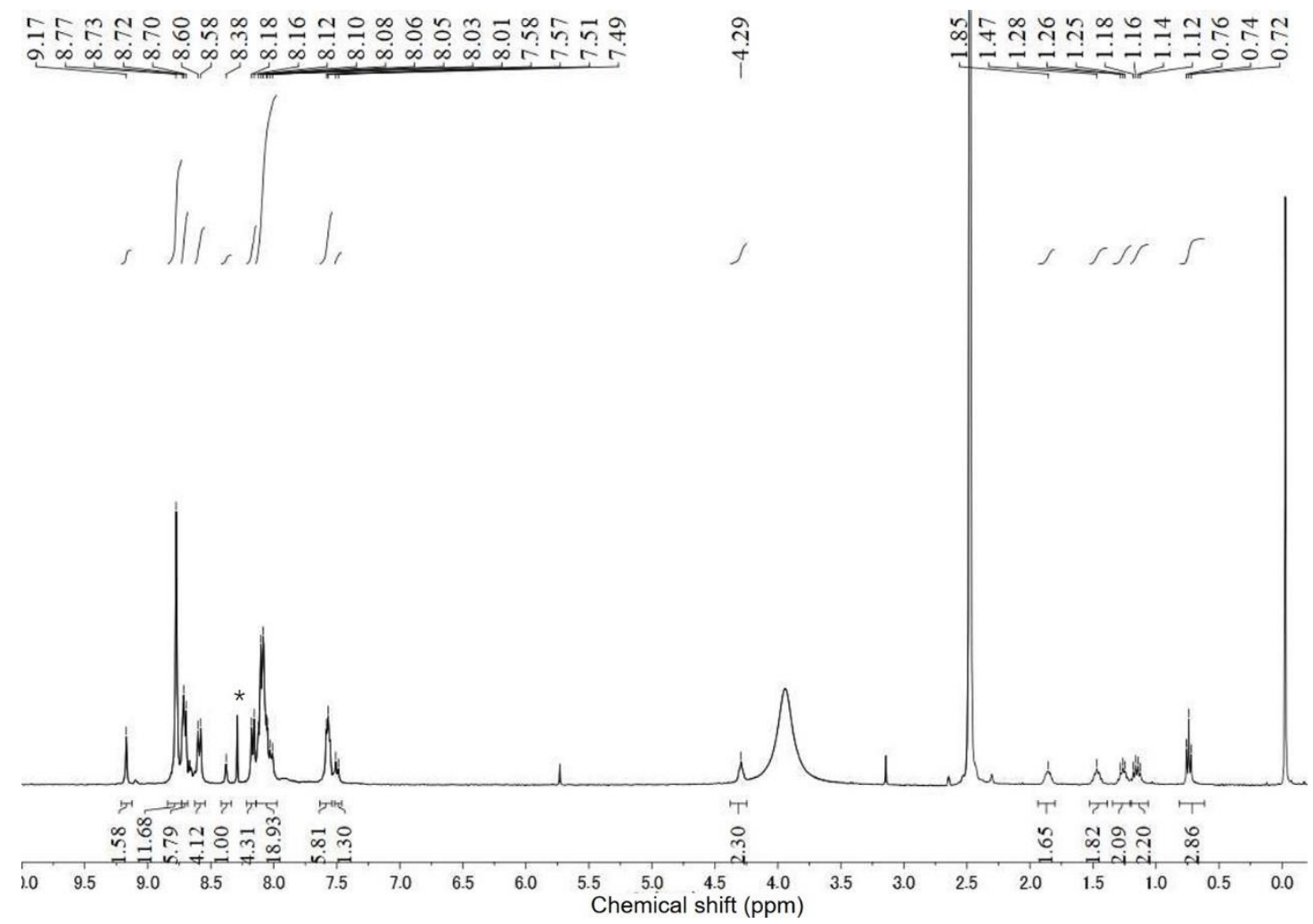

Figure S9: ${ }^{1} \mathrm{H}$ NMR $\left(400 \mathrm{MHz}, d_{6}\right.$-DMSO, $\left.300 \mathrm{~K}\right)$ spectrum of Compound 7. * represents the solvent residue of $\mathrm{CHCl}_{3}$.

भุक

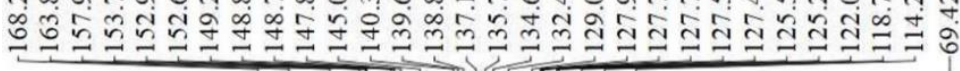

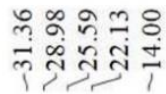

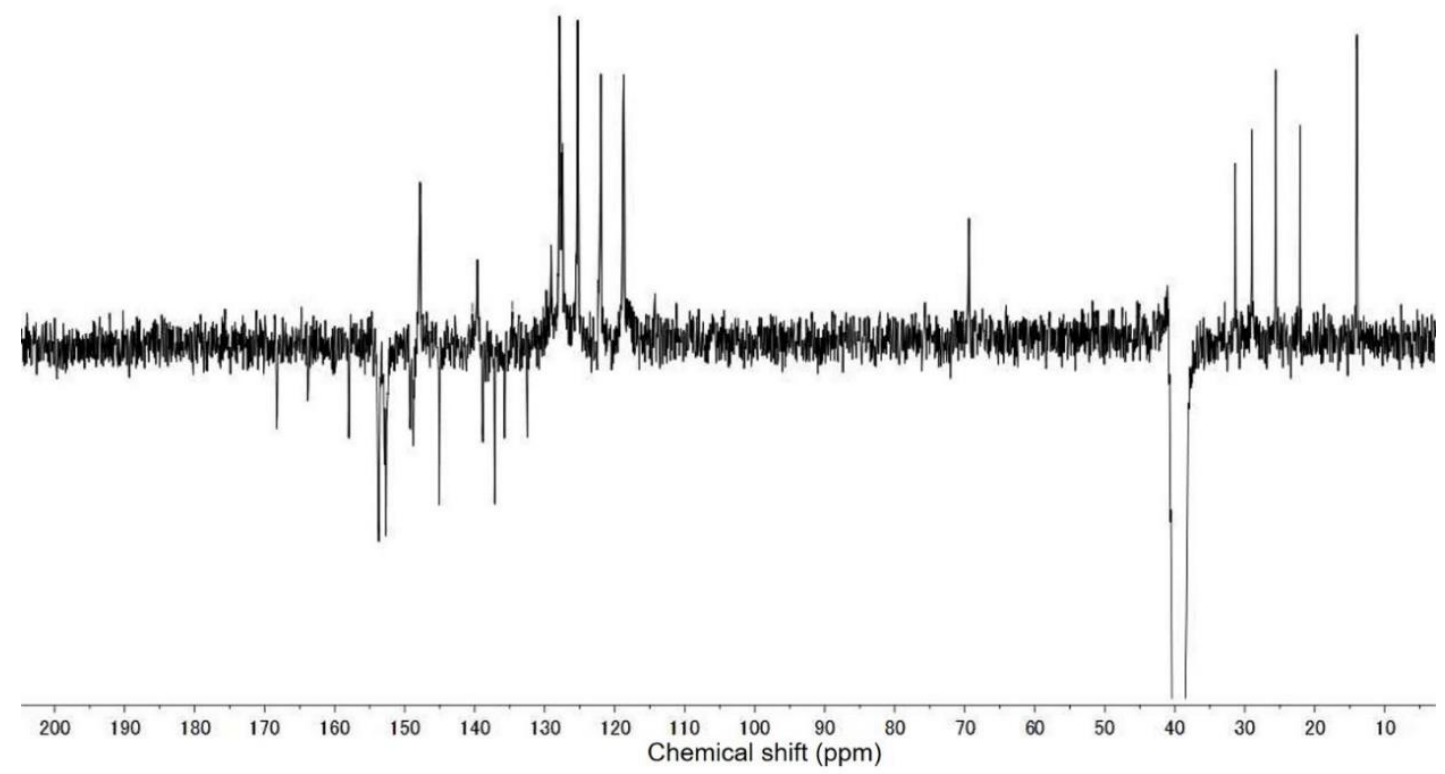

Figure S10: DEPTQ ${ }^{13} \mathrm{C}$ NMR $\left(125 \mathrm{MHz}, d_{6}\right.$-DMSO, $\left.300 \mathrm{~K}\right)$ spectrum of Compound 7. 


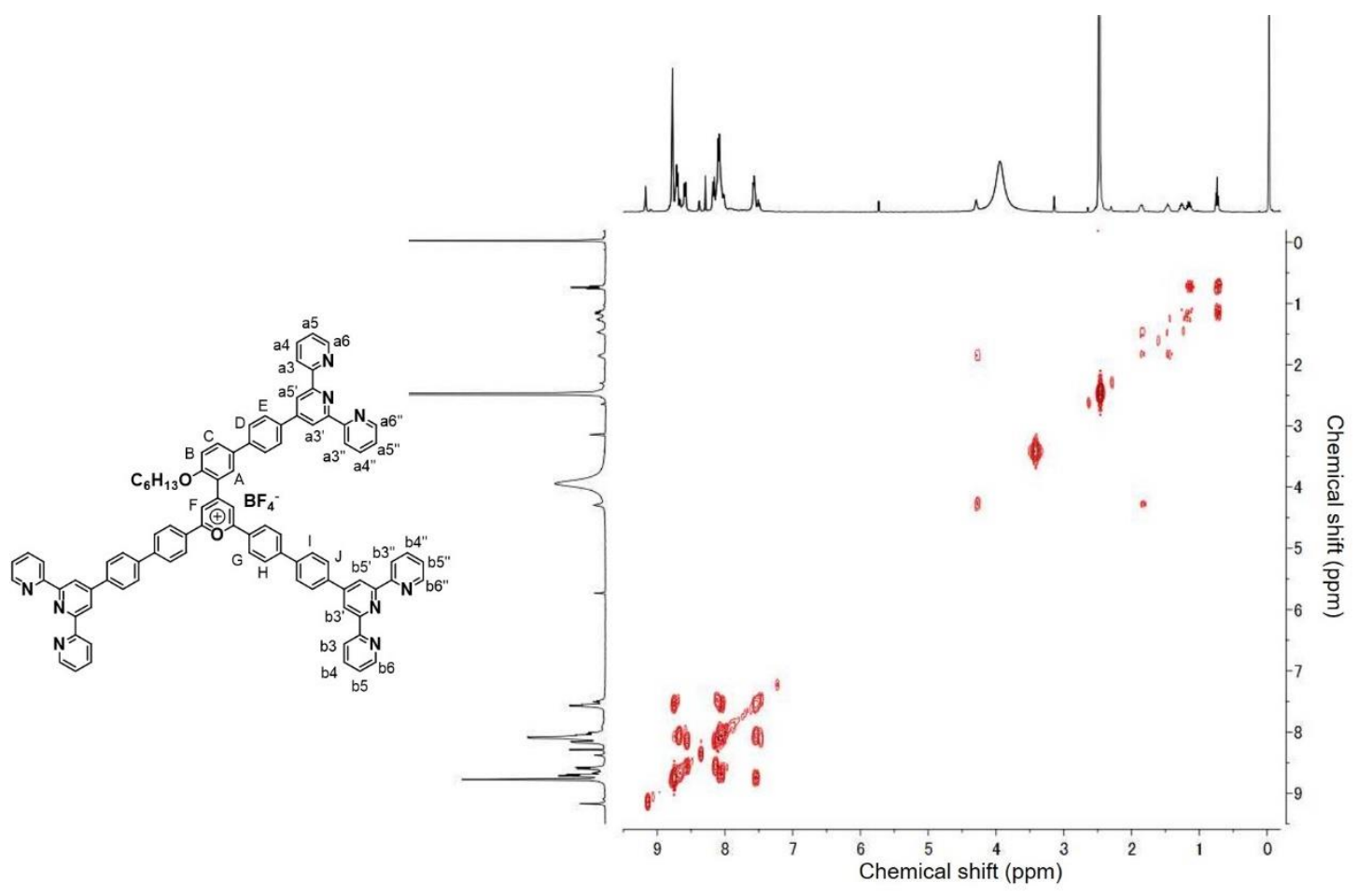

Figure S11: 2D COSY NMR (400 MHz, $\left.d_{6}-\mathrm{DMSO}, 300 \mathrm{~K}\right)$ spectrum of Compound 7.

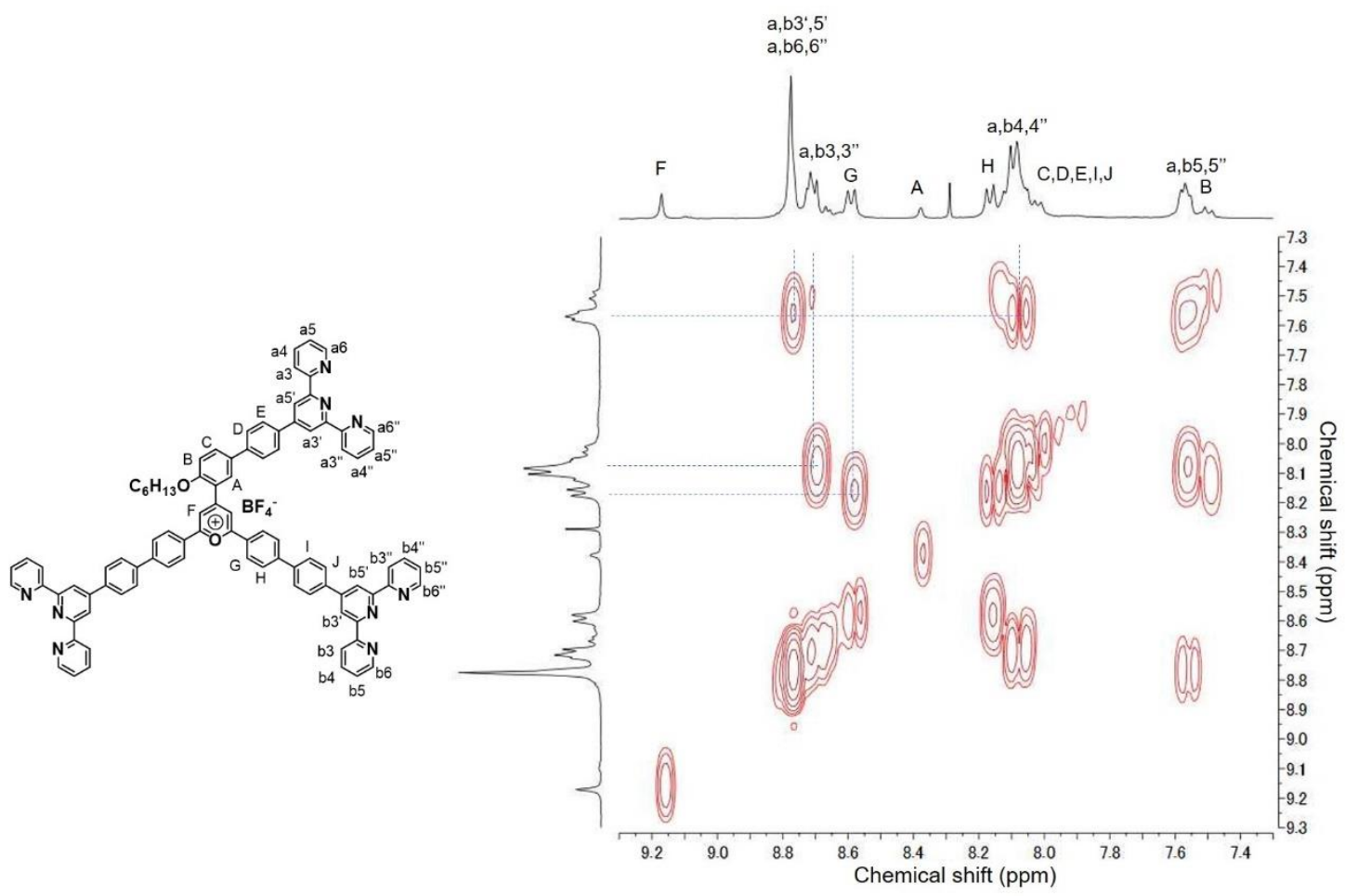

Figure S12: 2D COSY NMR (400 MHz, $d_{6}$-DMSO, $\left.300 \mathrm{~K}\right)$ spectrum of Compound 7 (aromatic region). 


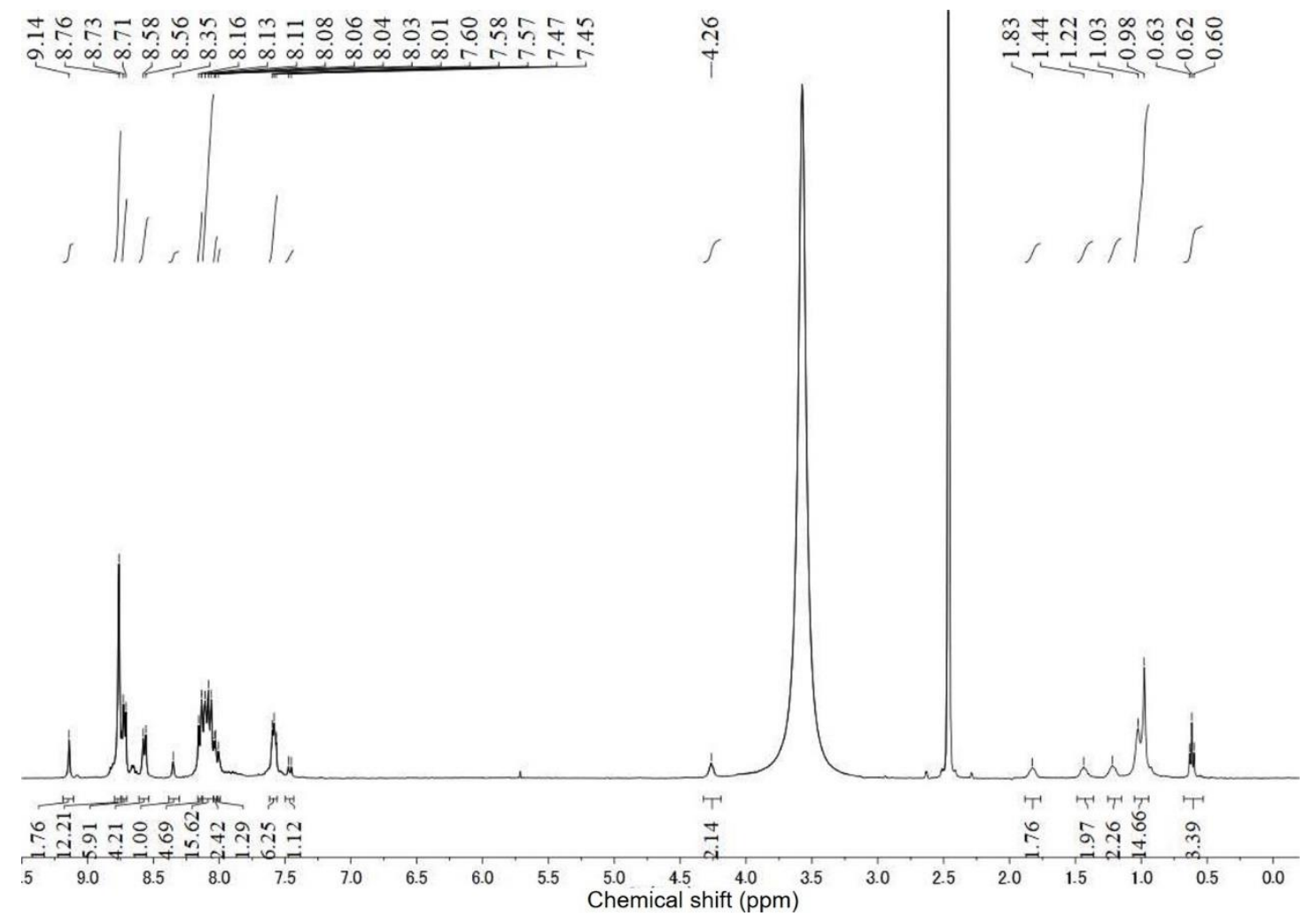

Figure S13: ${ }^{1} \mathrm{H}$ NMR (400 MHz, $d_{6}$-DMSO, $\left.300 \mathrm{~K}\right)$ spectrum of Compound 8.

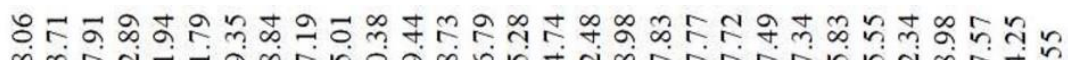

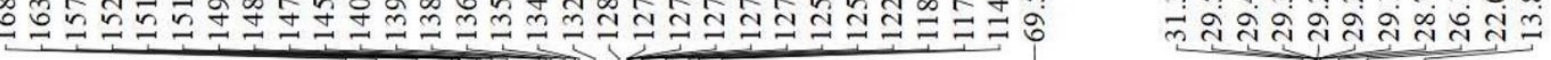

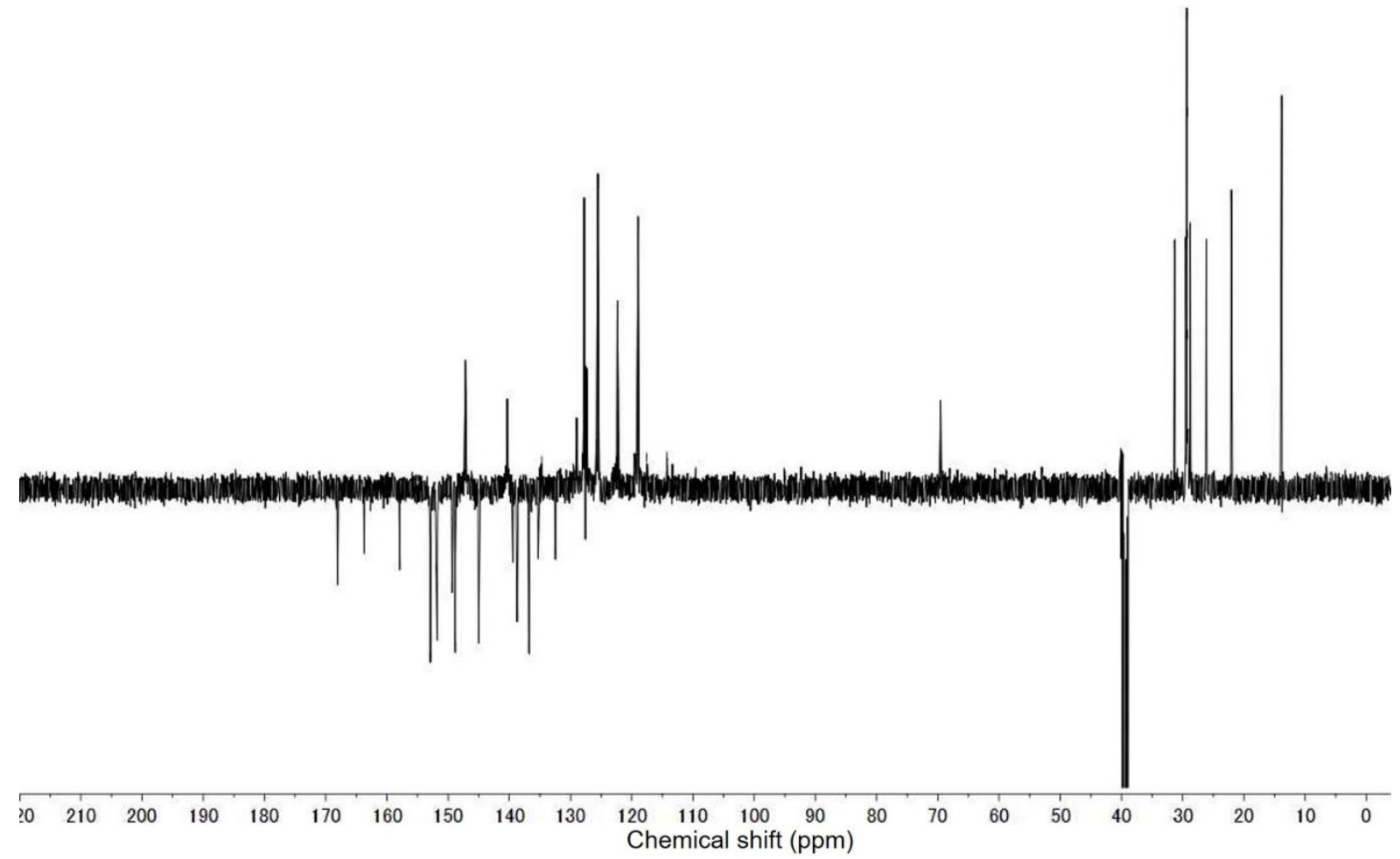

Figure S14: DEPTQ ${ }^{13} \mathrm{C}$ NMR (125 MHz, $d_{6}$-DMSO, $\left.300 \mathrm{~K}\right)$ spectrum of Compound 8. 


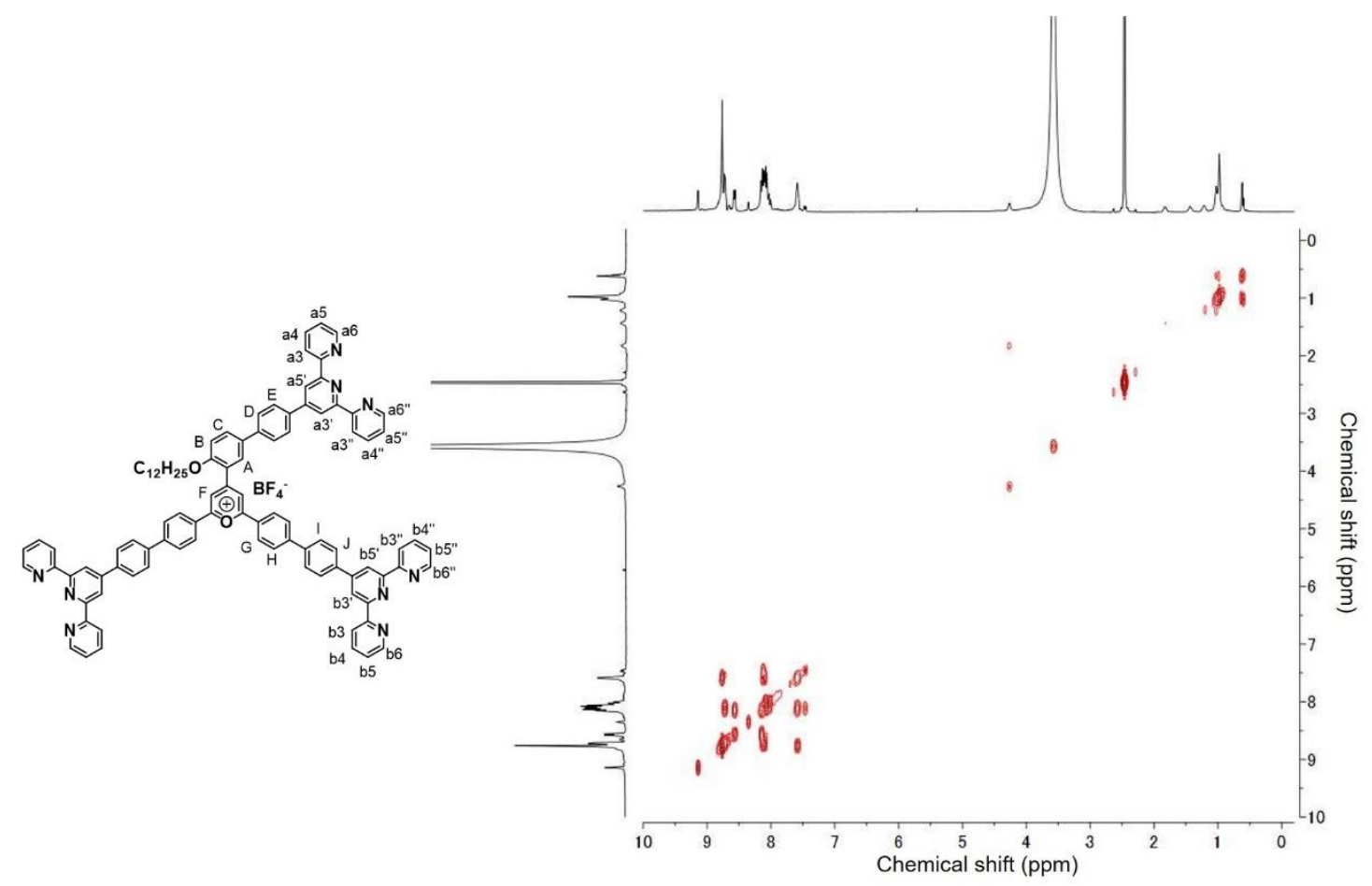

Figure S15: 2D COSY NMR (400 MHz, $\left.d_{6}-\mathrm{DMSO}, 300 \mathrm{~K}\right)$ spectrum of Compound 8.

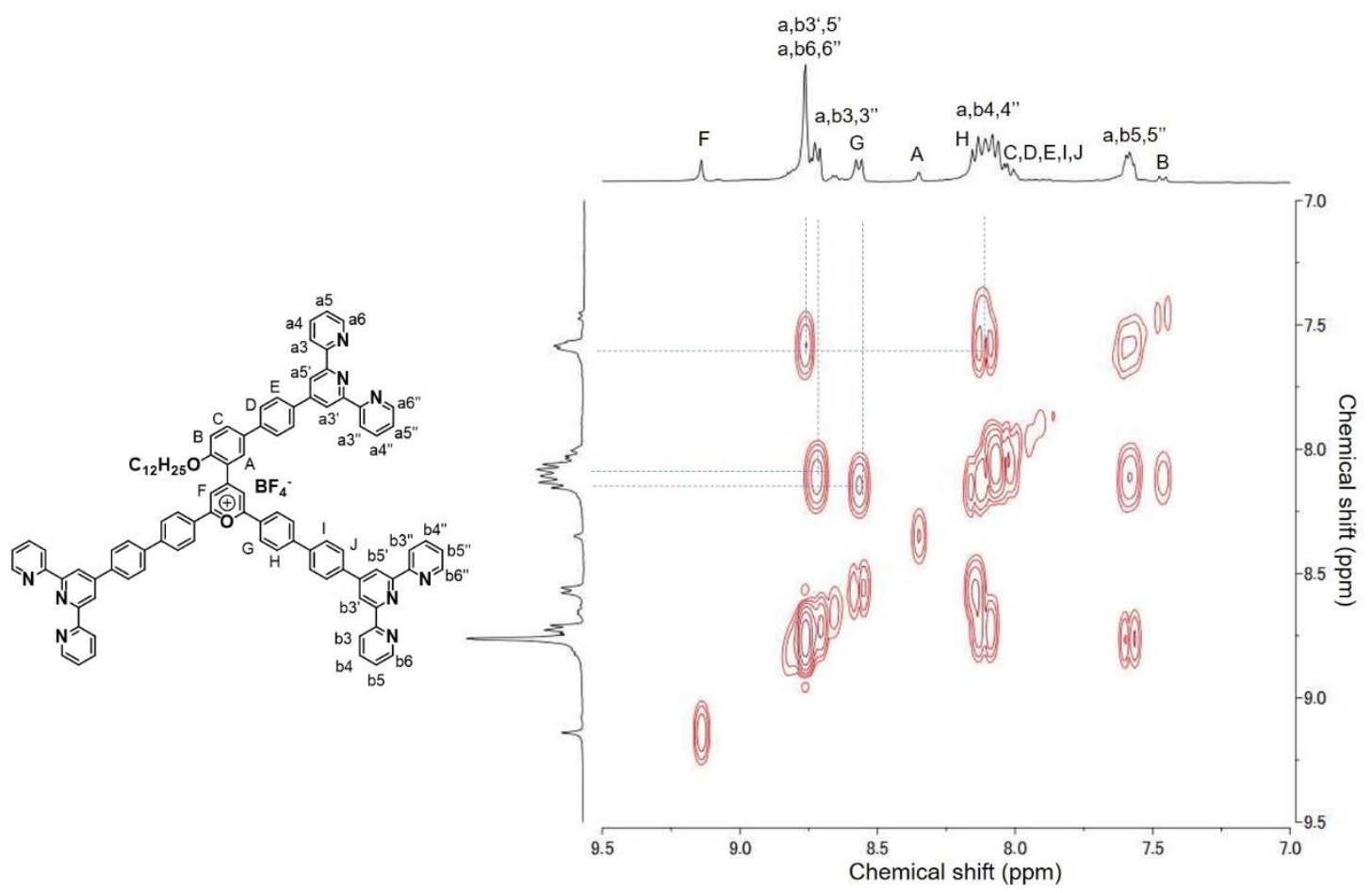

Figure S16: 2D COSY NMR (400 MHz, $d_{6}$-DMSO, $300 \mathrm{~K}$ ) spectrum of Compound 8 (aromatic region). 


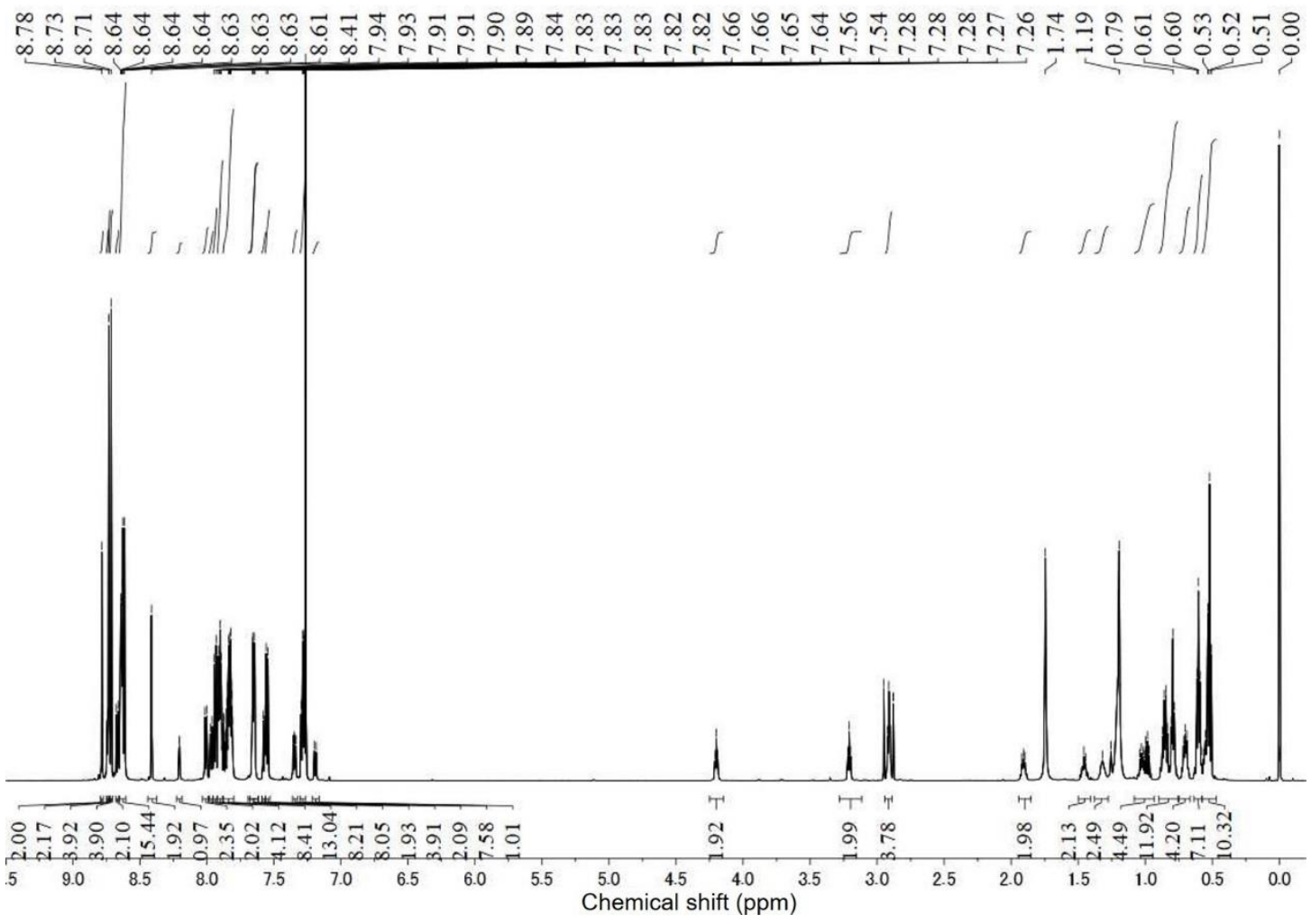

Figure S17: ${ }^{1} \mathrm{H}$ NMR $\left(600 \mathrm{MHz}, \mathrm{CDCl}_{3}, 300 \mathrm{~K}\right)$ spectrum of Ligand $\mathbf{L 1}$.

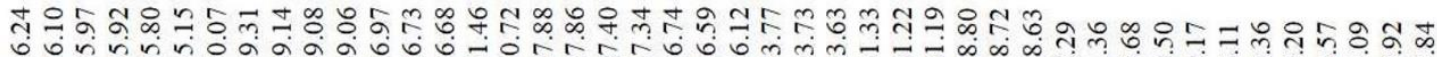

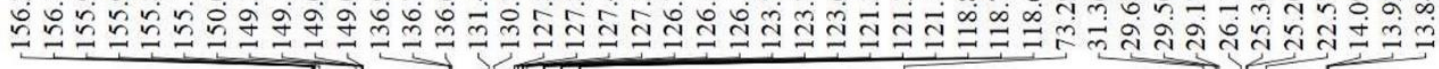

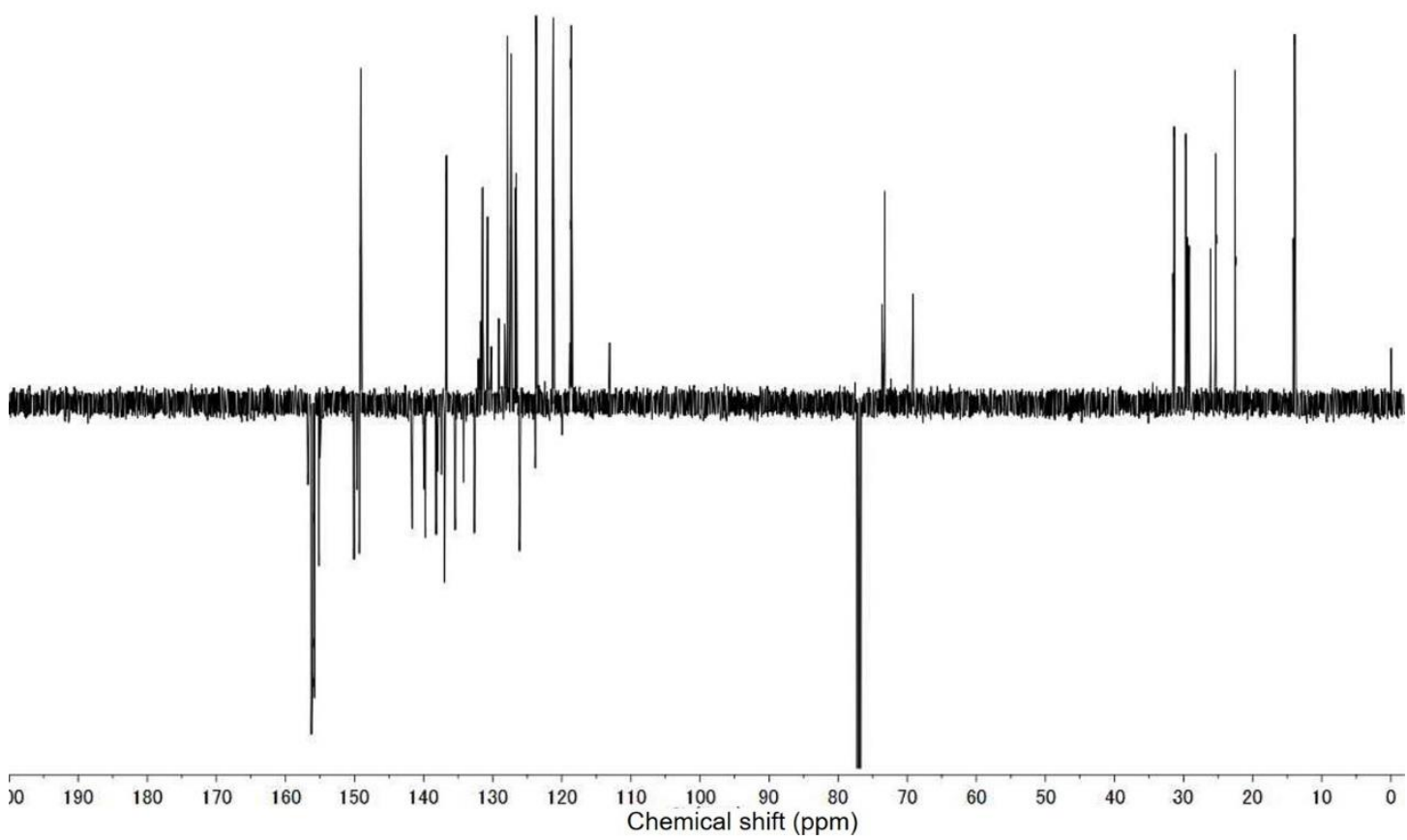

Figure S18: DEPTQ ${ }^{13} \mathrm{C}$ NMR $\left(125 \mathrm{MHz}, \mathrm{CDCl}_{3}, 300 \mathrm{~K}\right)$ spectrum of Ligand L1. 


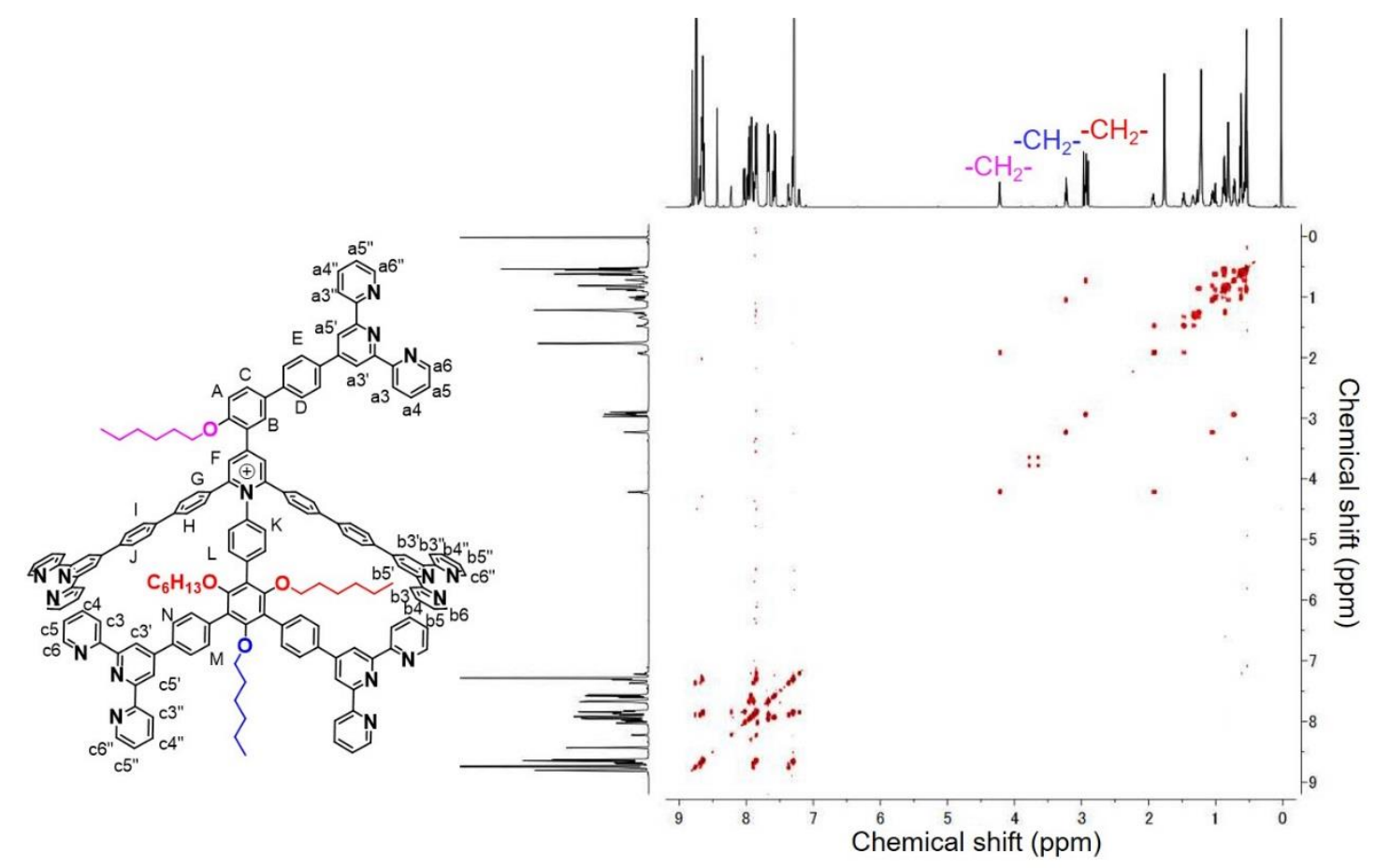

Figure S19: 2D COSY NMR (600 MHz, $\left.\mathrm{CDCl}_{3}, 300 \mathrm{~K}\right)$ spectrum of Ligand $\mathbf{L 1}$.

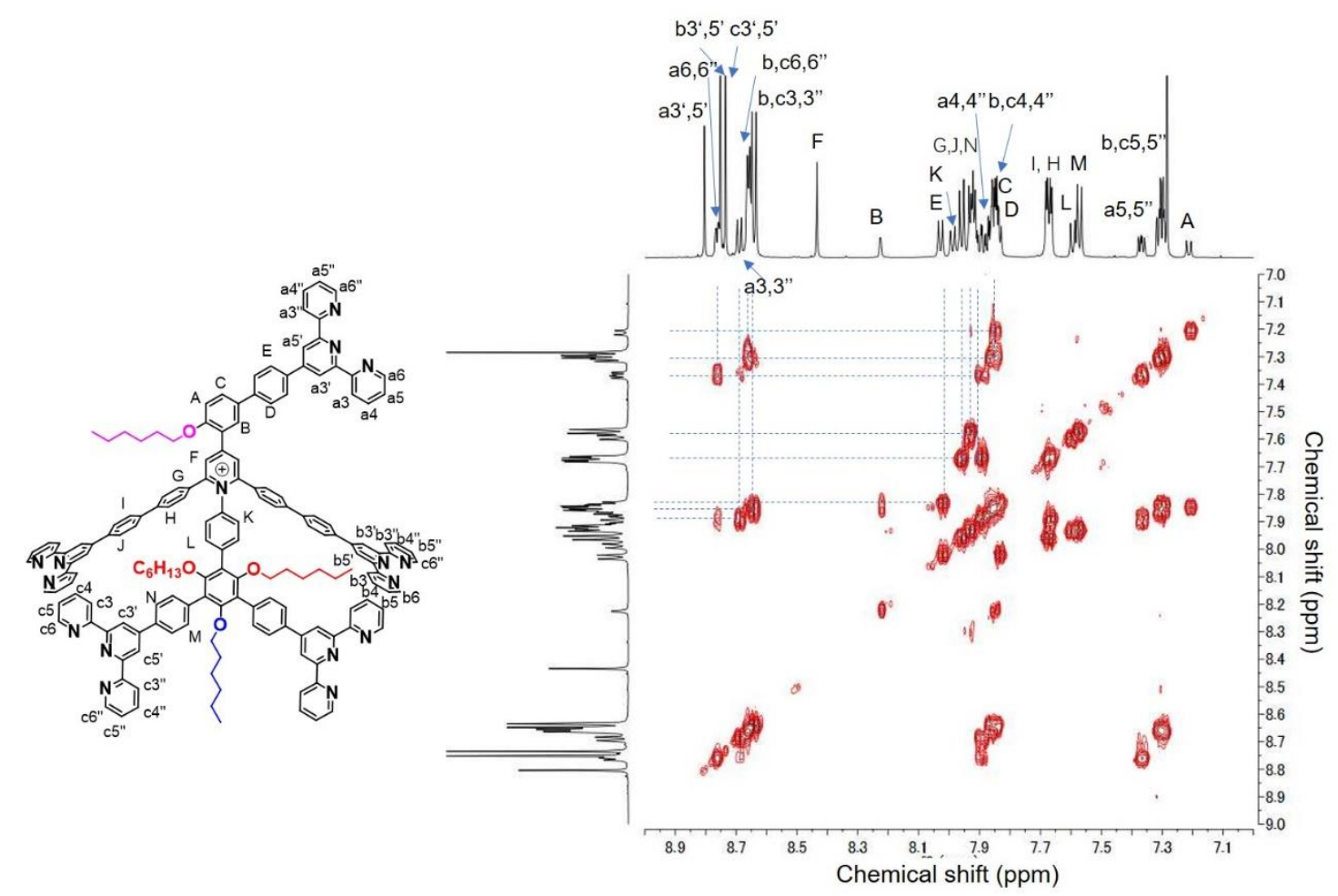

Figure S20: 2D COSY NMR (600 MHz, $\left.\mathrm{CDCl}_{3}, 300 \mathrm{~K}\right)$ spectrum of Ligand L1 (aromatic region). 


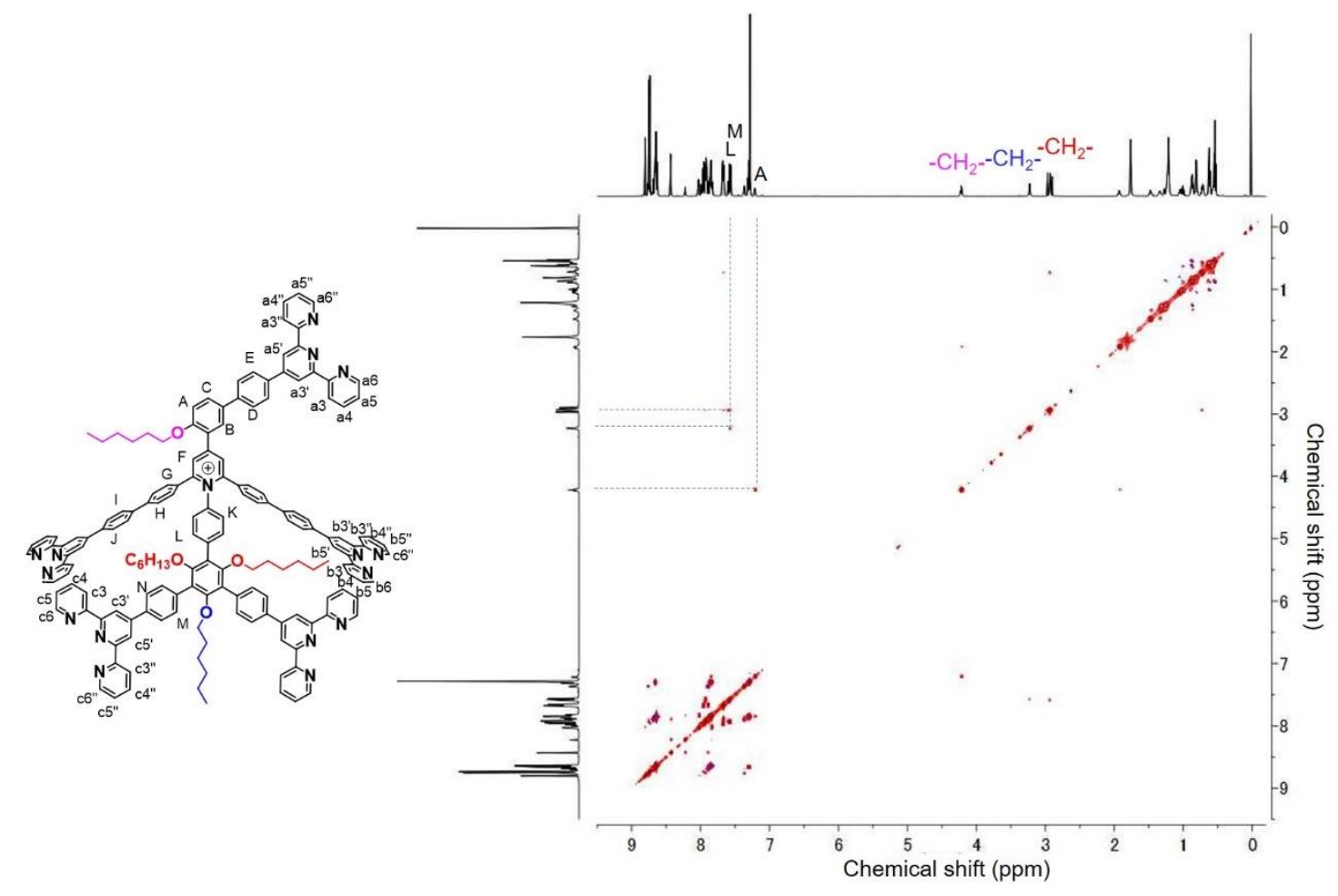

Figure S21: 2D NOESY NMR (600 MHz, $\left.\mathrm{CDCl}_{3}, 300 \mathrm{~K}\right)$ spectrum of Ligand $\mathbf{L 1}$.

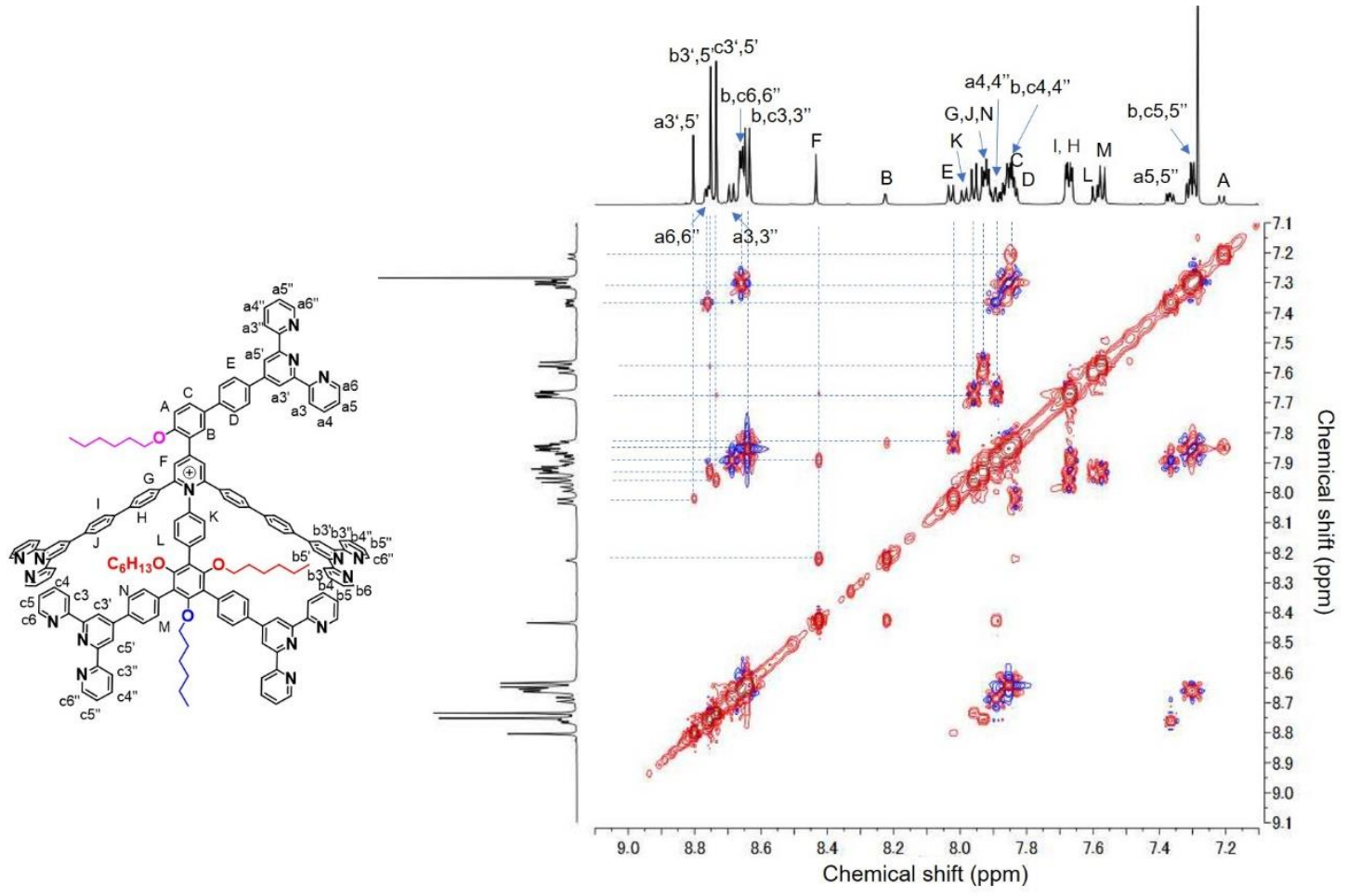

Figure S22: 2D NOESY NMR (600 MHz, $\left.\mathrm{CDCl}_{3}, 300 \mathrm{~K}\right)$ spectrum of Ligand $\mathbf{L 1}$ (aromatic region). 


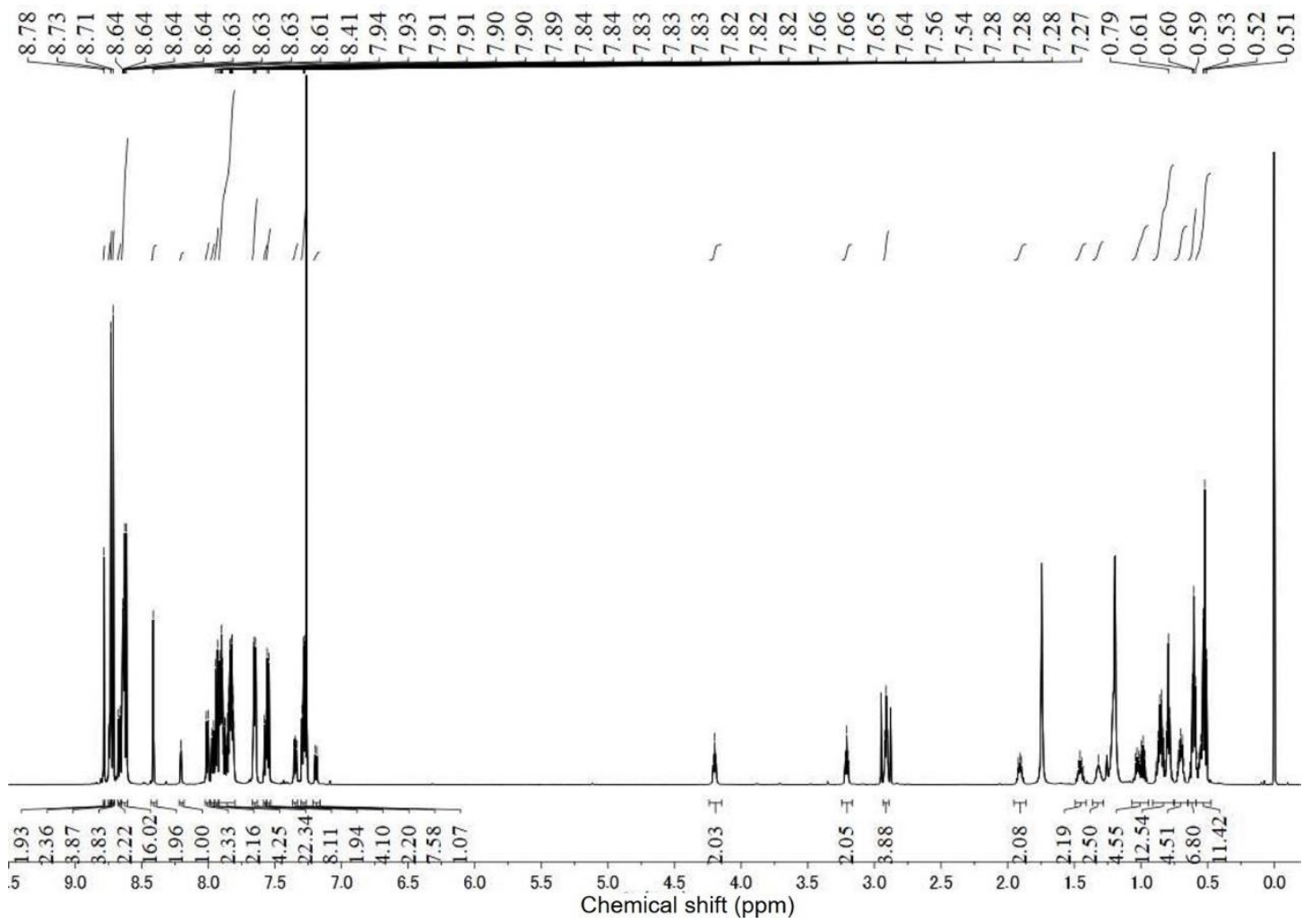

Figure S23: ${ }^{1} \mathrm{H}$ NMR $\left(600 \mathrm{MHz}, \mathrm{CDCl}_{3}, 300 \mathrm{~K}\right)$ spectrum of Ligand $\mathbf{L 2}$.

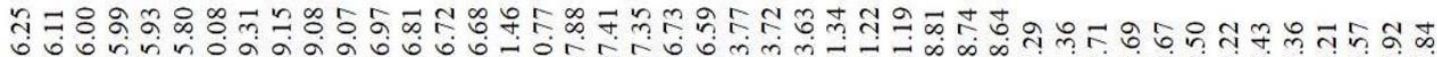

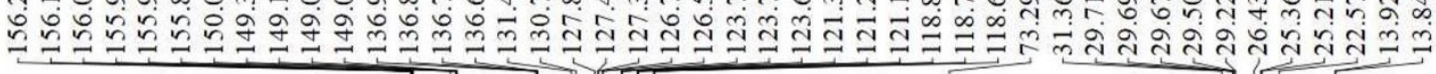

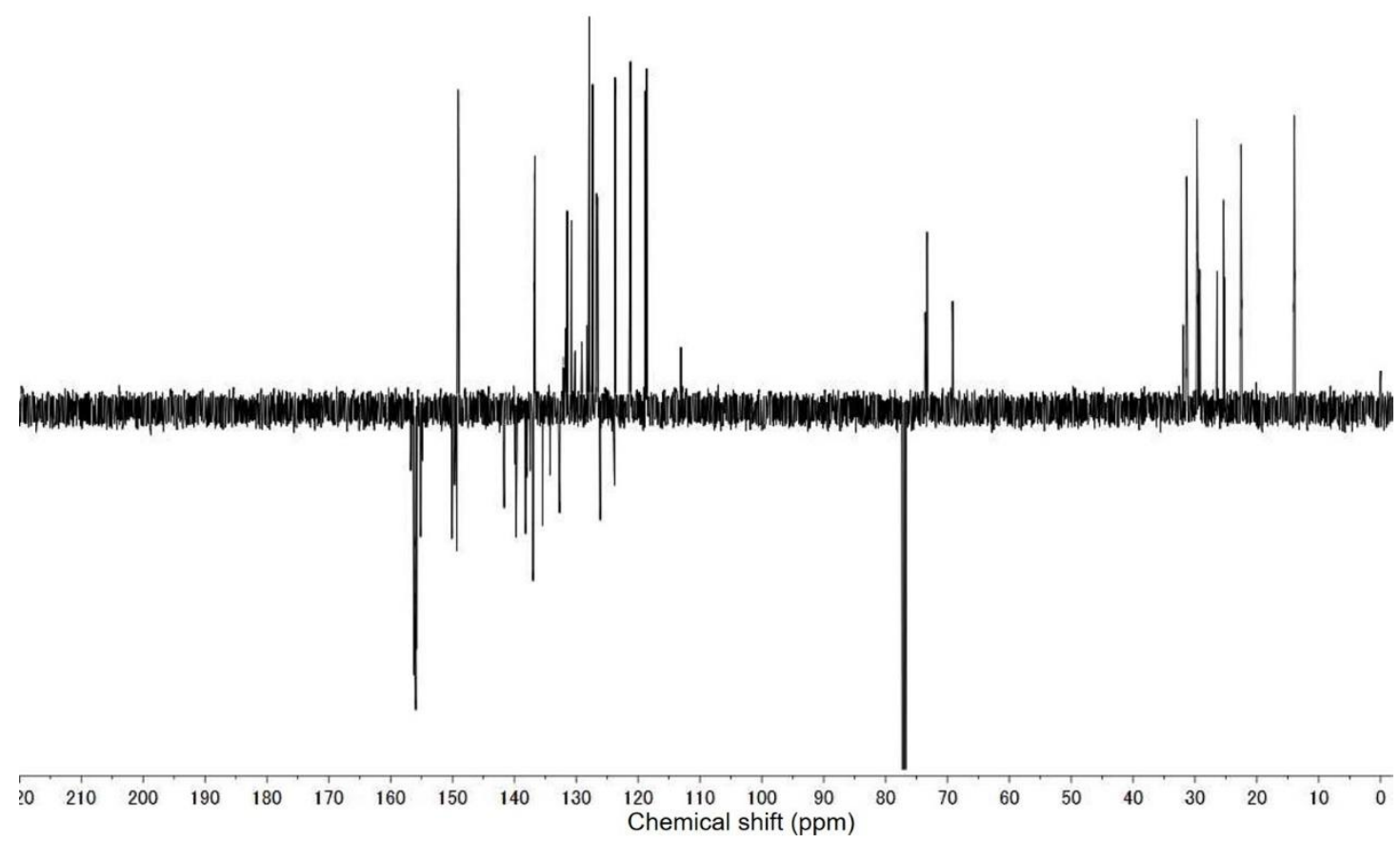

Figure S24: DEPTQ ${ }^{13} \mathrm{C}$ NMR $\left(125 \mathrm{MHz}, \mathrm{CDCl}_{3}, 300 \mathrm{~K}\right)$ spectrum of Ligand $\mathbf{L 2}$. 


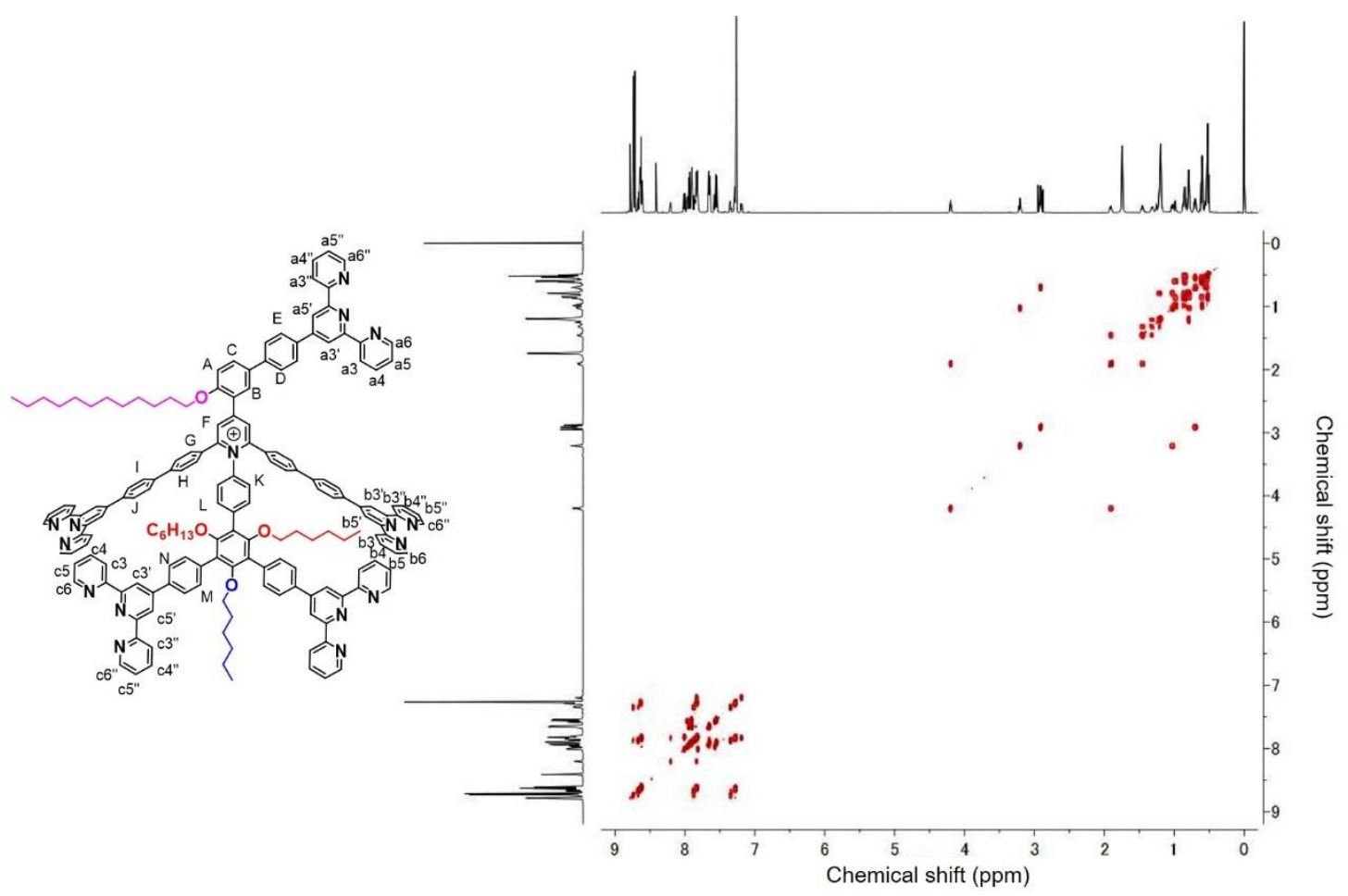

Figure S25: 2D COSY NMR (600 MHz, $\left.\mathrm{CDCl}_{3}, 300 \mathrm{~K}\right)$ spectrum of Ligand $\mathbf{L 2}$.

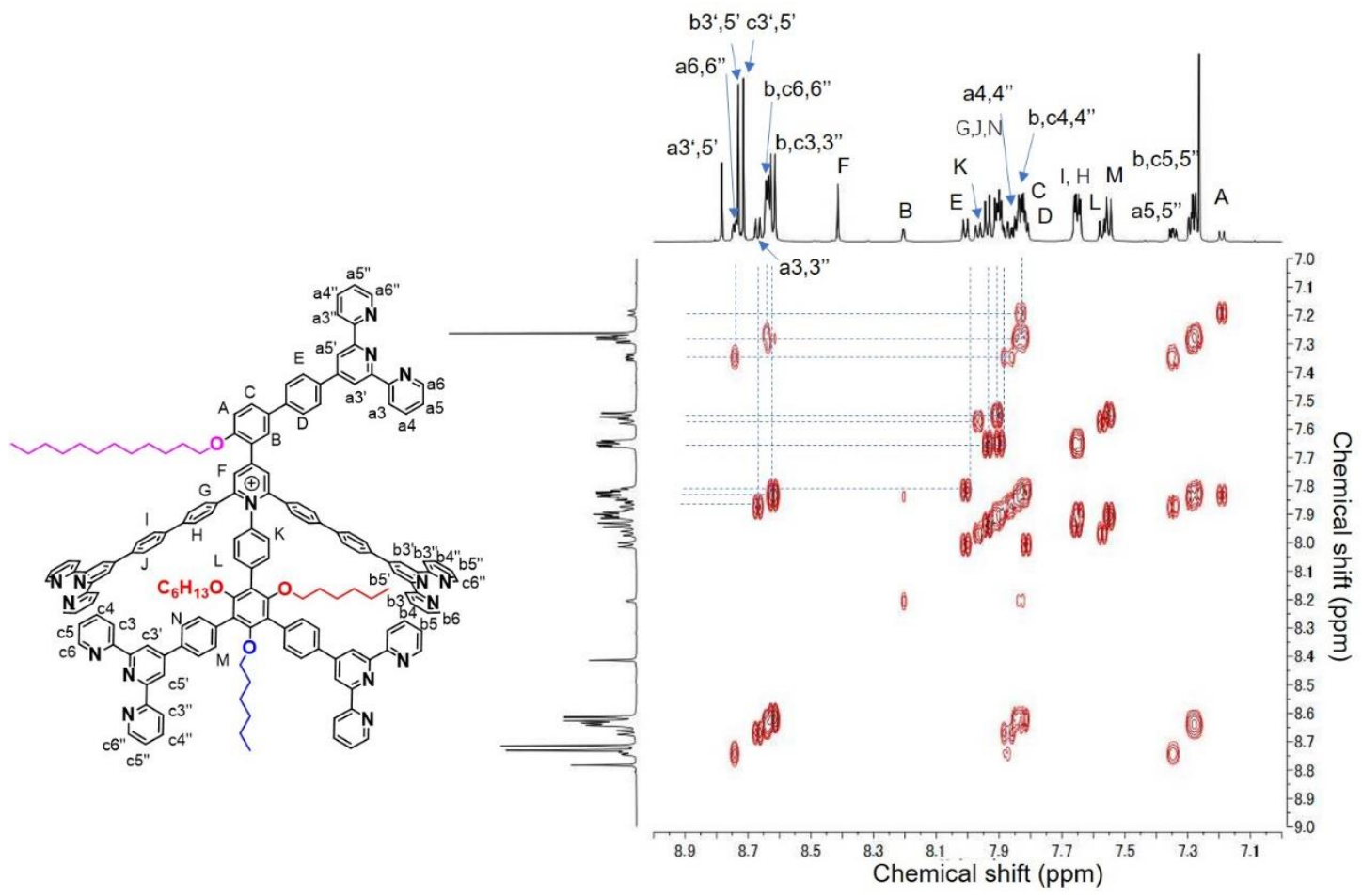

Figure S26: 2D COSY NMR (600 MHz, $\left.\mathrm{CDCl}_{3}, 300 \mathrm{~K}\right)$ spectrum of Ligand $\mathbf{L 2}$ (aromatic region). 


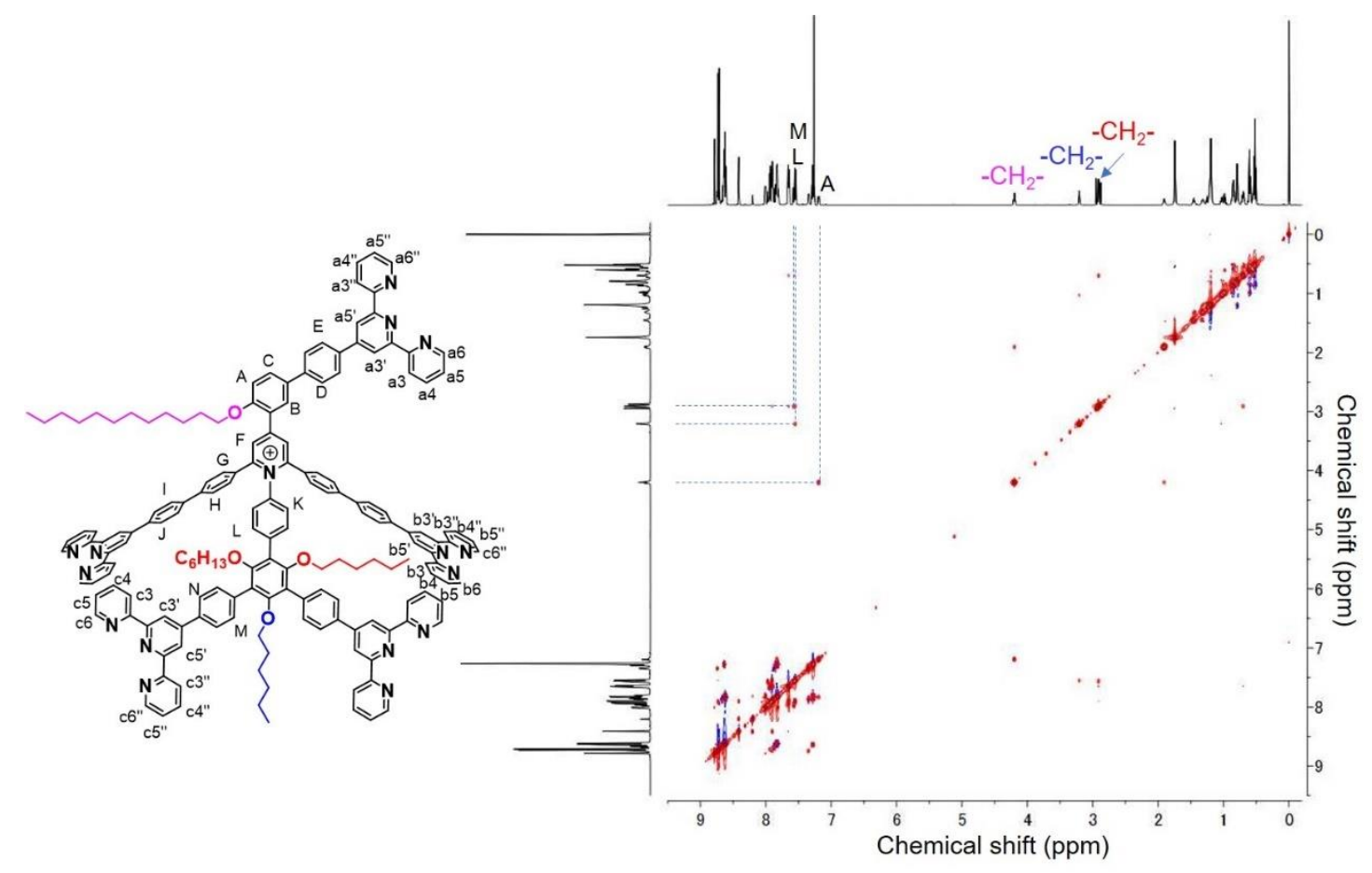

Figure S27: 2D NOESY NMR (600 MHz, $\left.\mathrm{CDCl}_{3}, 300 \mathrm{~K}\right)$ spectrum of Ligand $\mathbf{L 2}$.

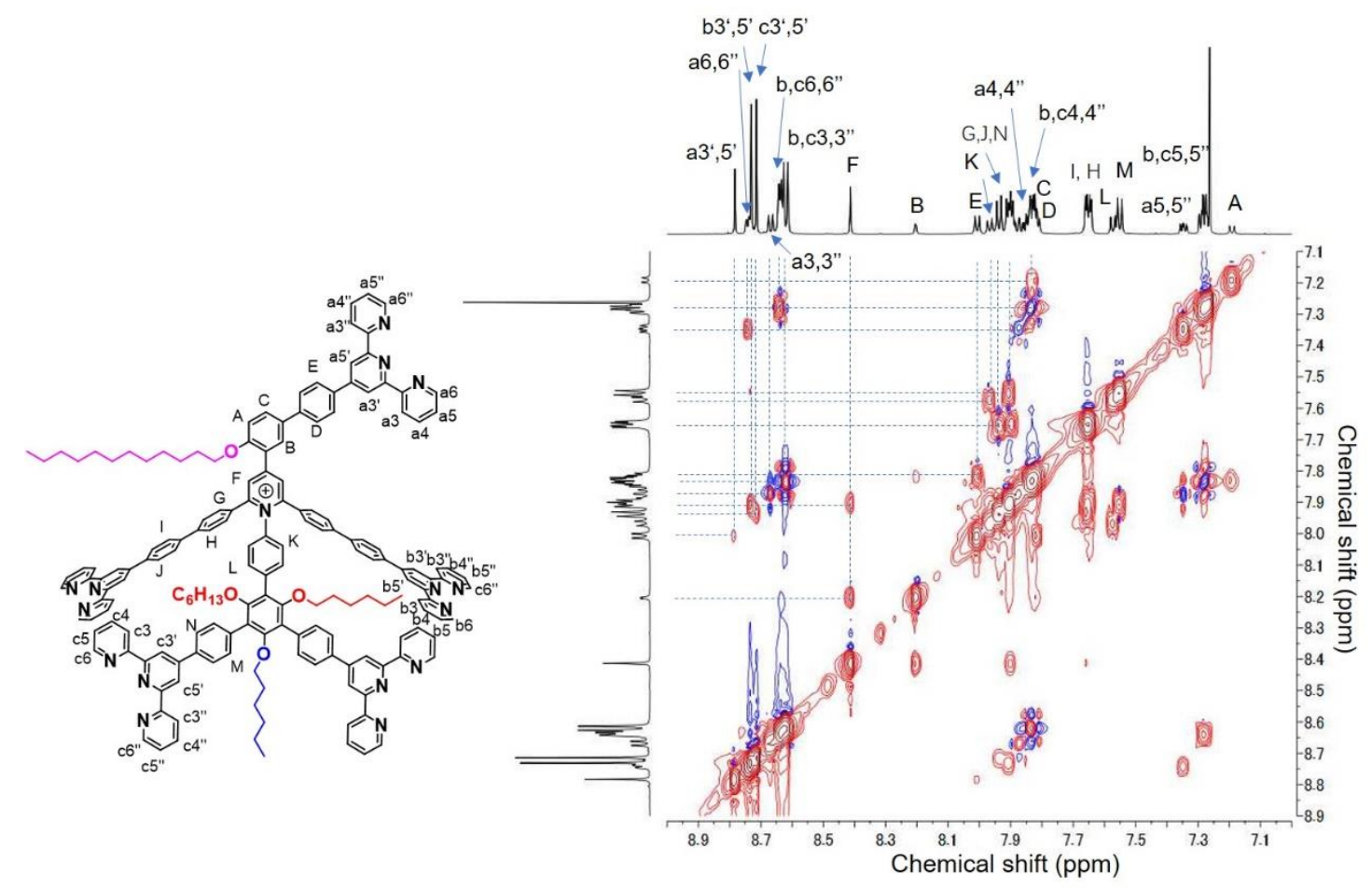

Figure S28: 2D NOESY NMR $\left(600 \mathrm{MHz}, \mathrm{CDCl}_{3}, 300 \mathrm{~K}\right)$ spectrum of Ligand $\mathbf{L 2}$ (aromatic region). 


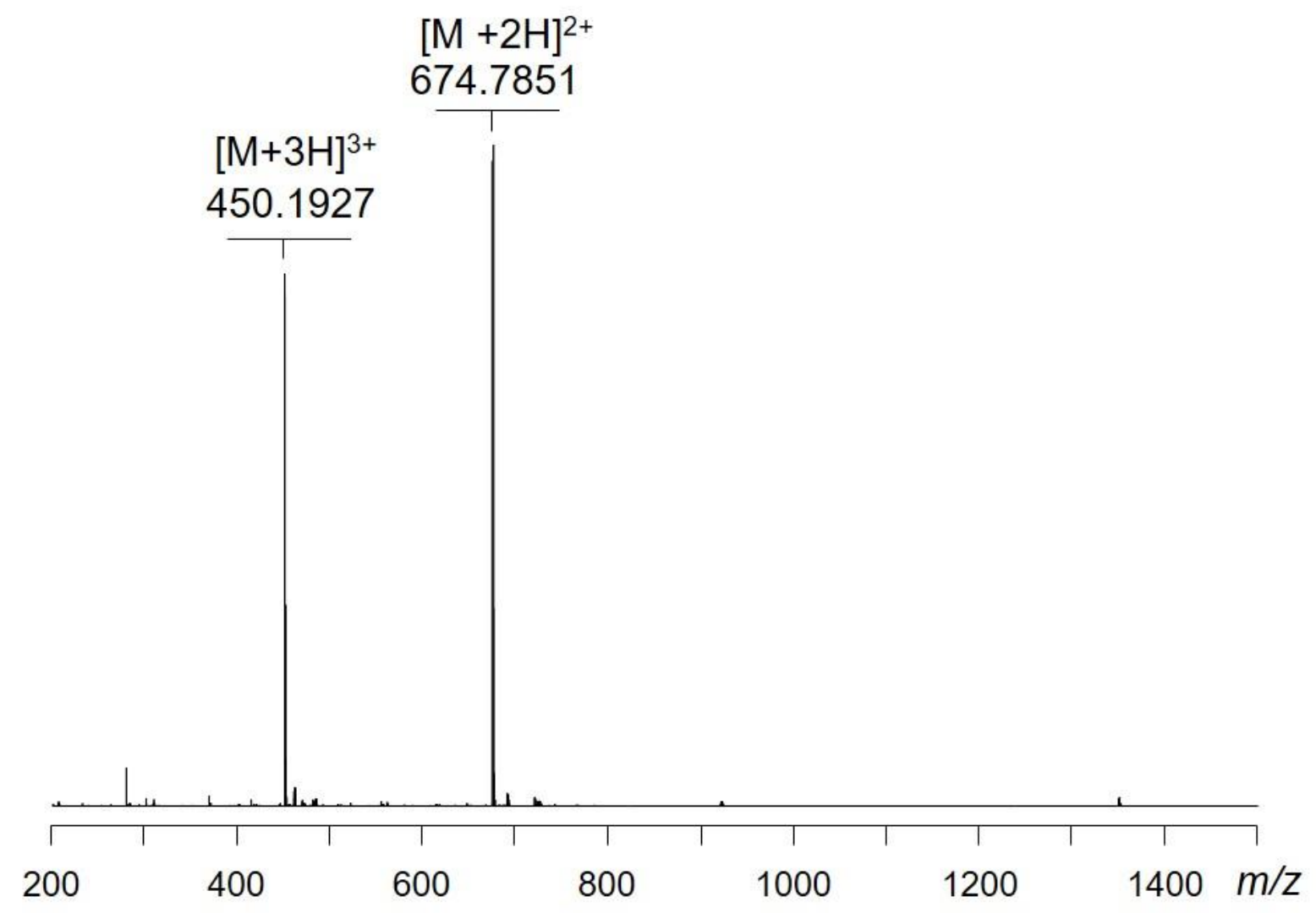

Figure S29: HR ESI-MS spectrum of 5 in $\mathrm{CHCl}_{3} / \mathrm{MeOH}(1 / 3)$.

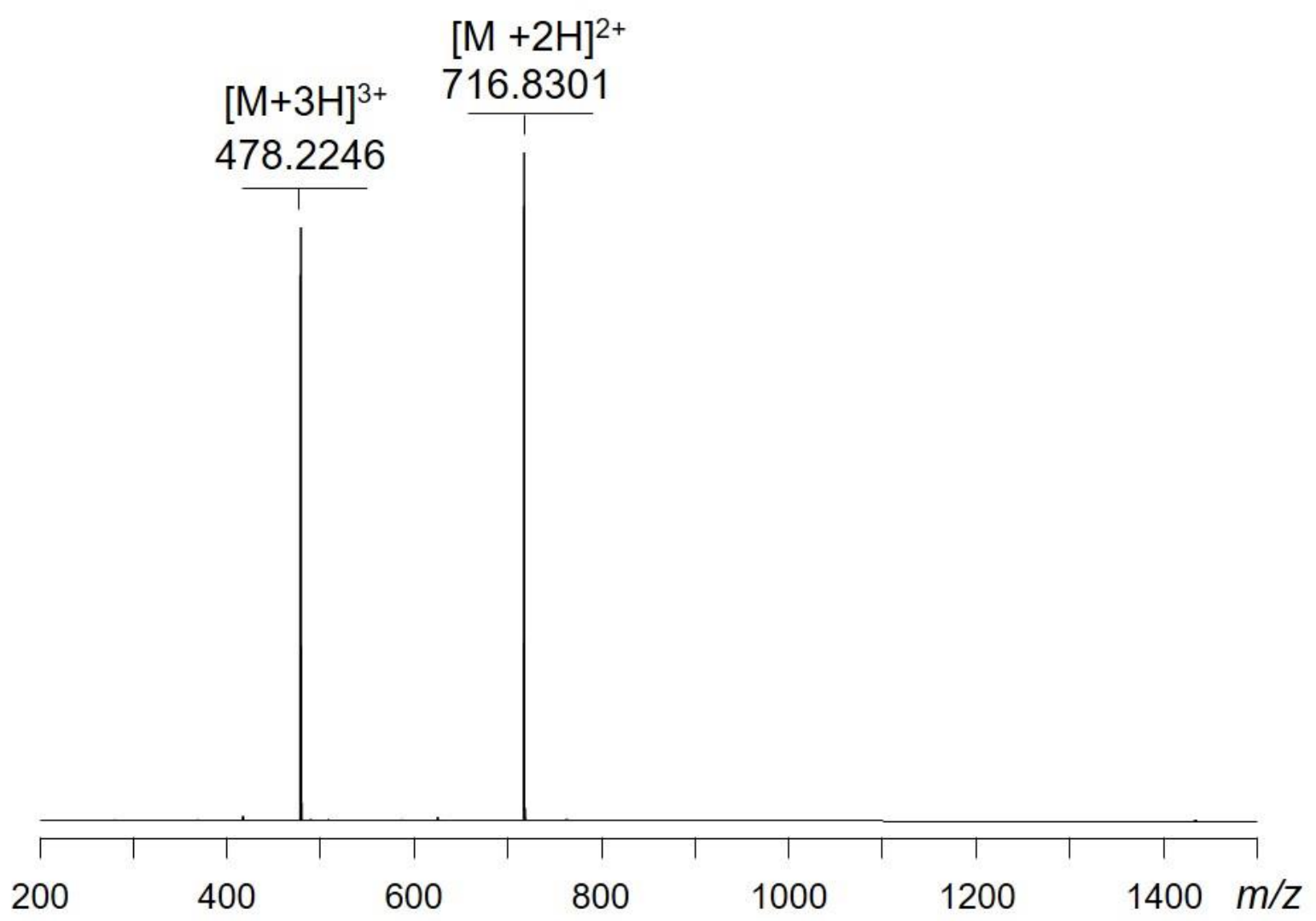

Figure S30: HR ESI-MS spectrum of 6 in $\mathrm{CHCl}_{3} / \mathrm{MeOH}(1 / 3)$. 


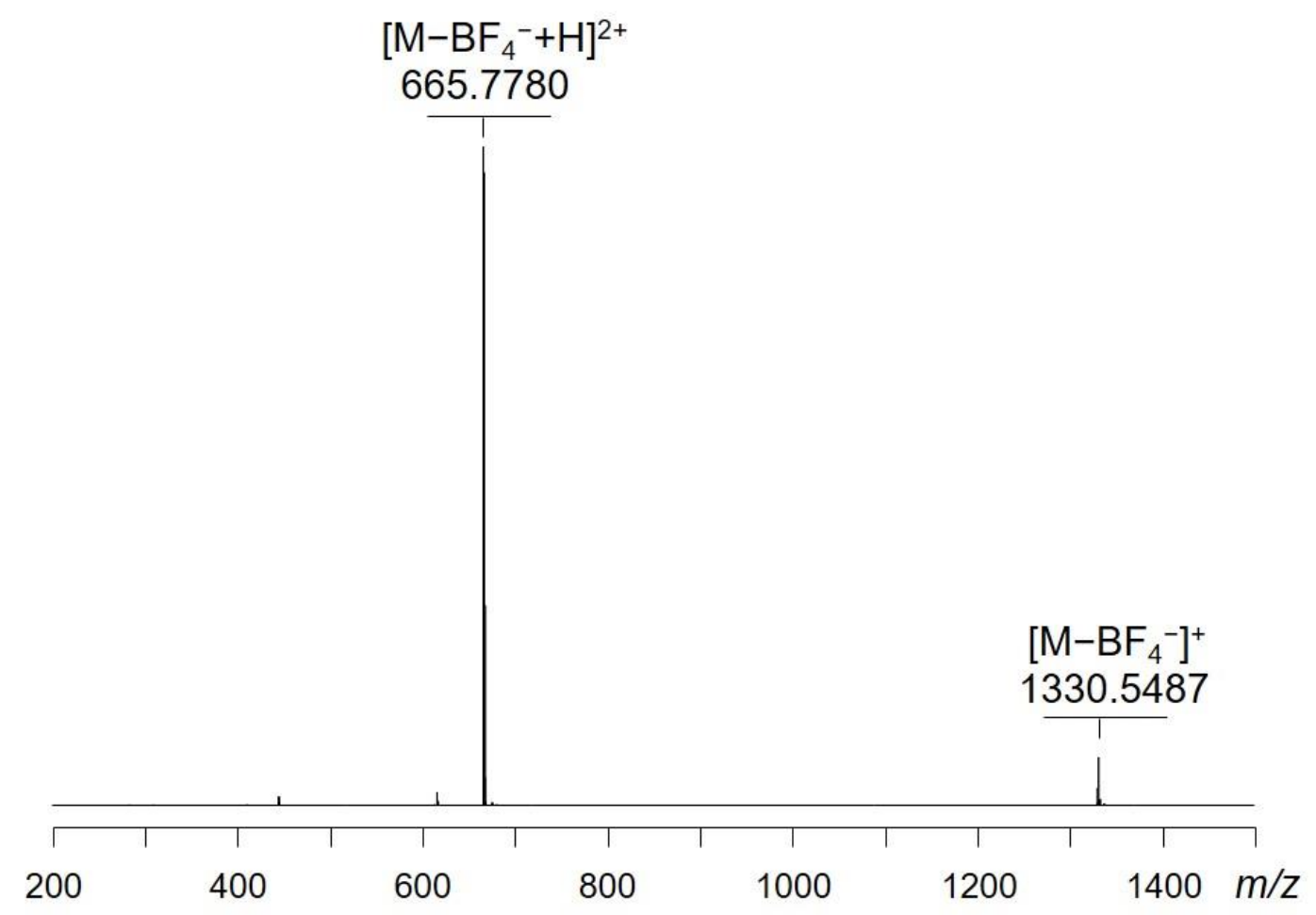

Figure S31: HR ESI-MS spectrum of 7 in DMSO/Acetone/MeOH (1/10/30).

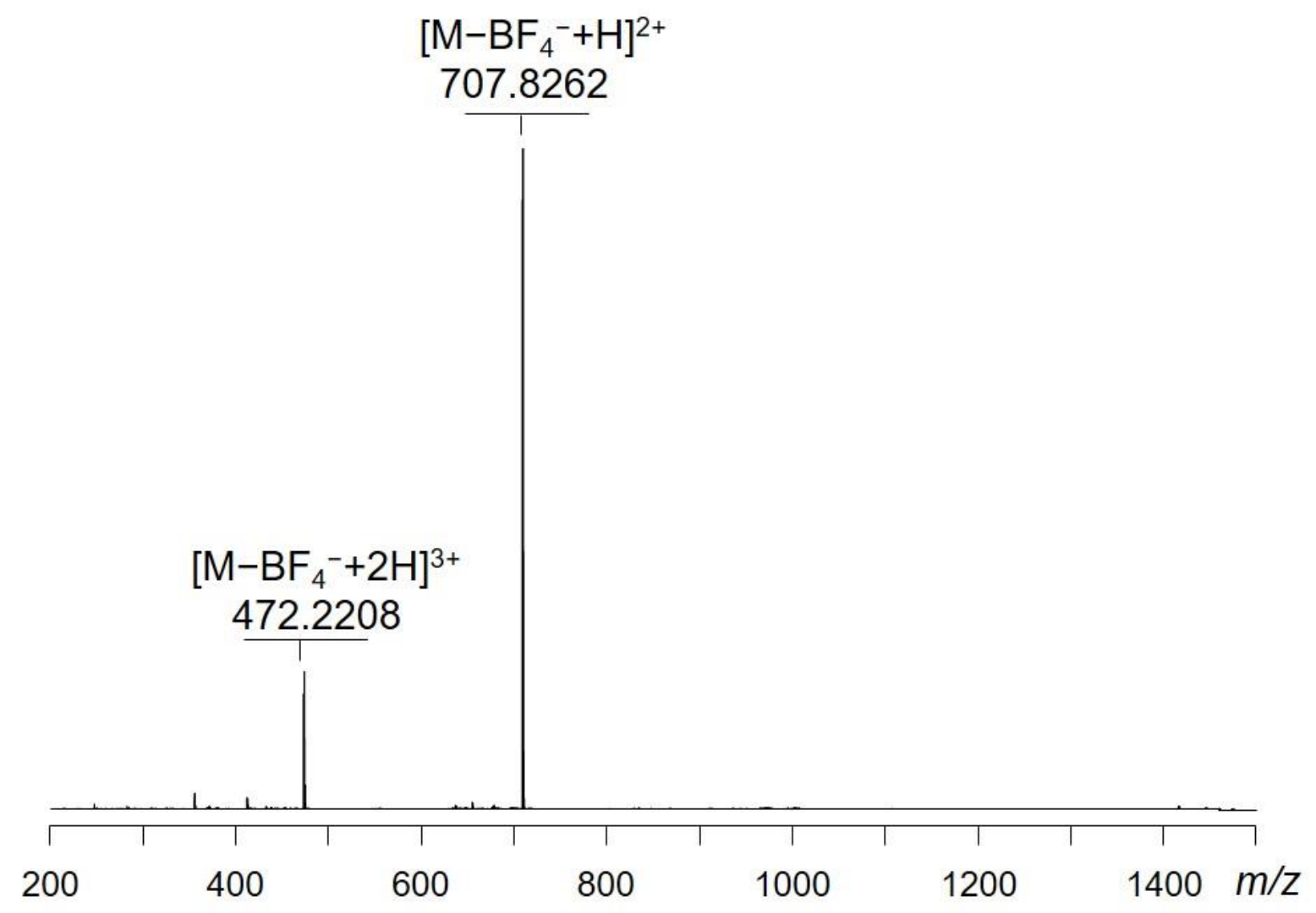

Figure S32: HR ESI-MS spectrum of 8 in DMSO/Acetone/MeOH (1/10/30). 


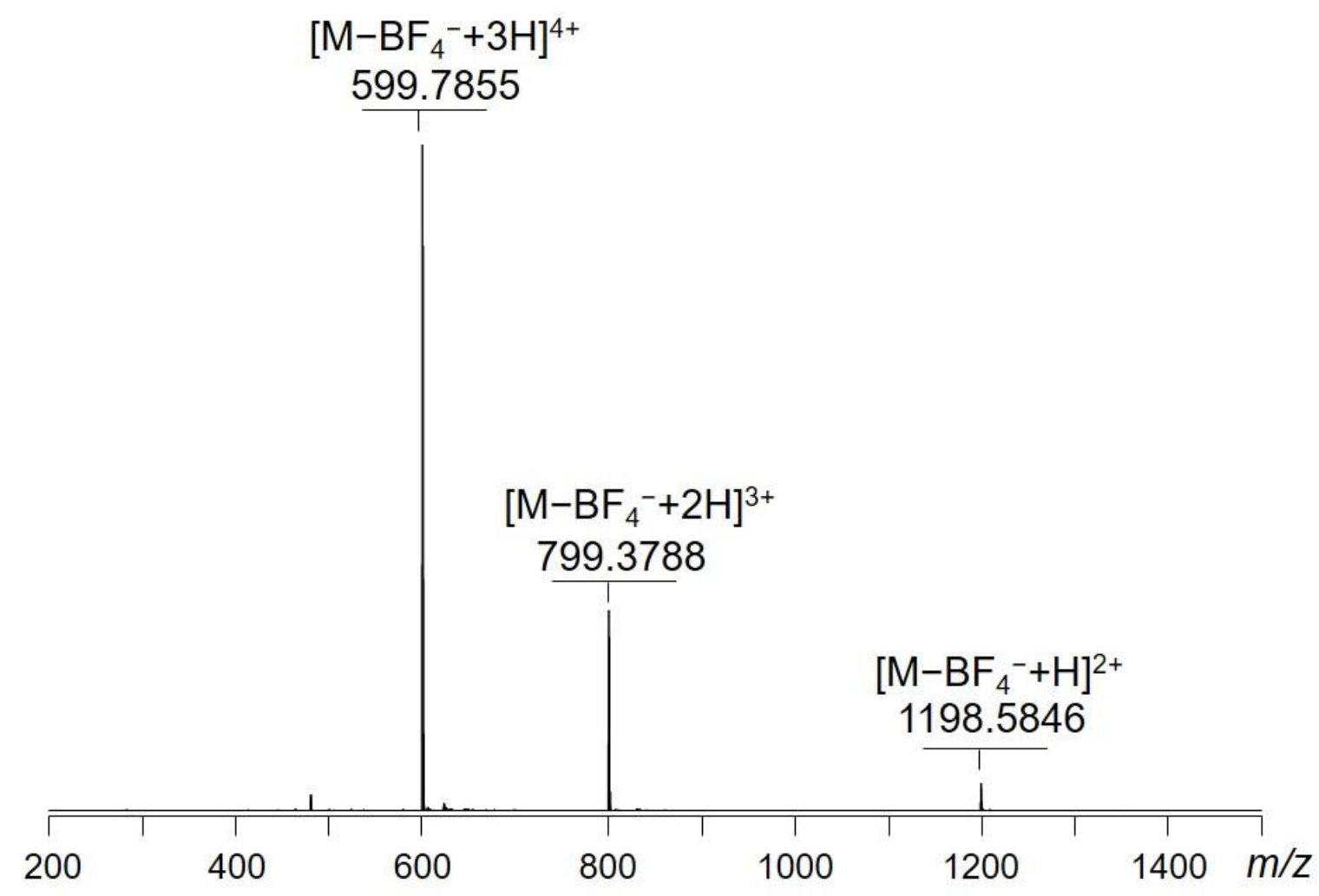

Figure S33: HR ESI-MS spectrum of Ligand $\mathbf{L 1}$ in $\mathrm{CHCl}_{3} / \mathrm{MeOH}(1 / 3)$.

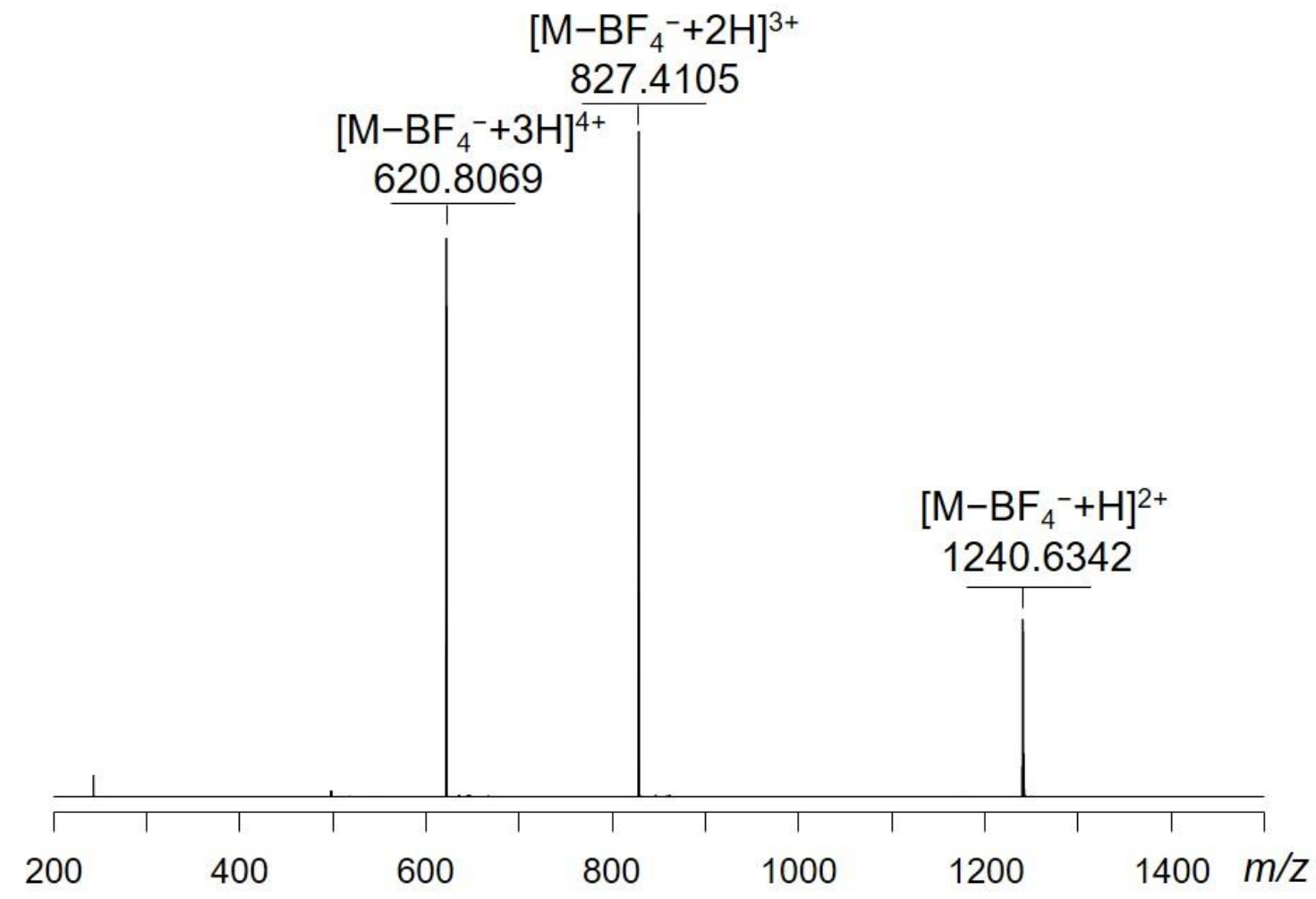

Figure S34: HR ESI-MS spectrum of Ligand $\mathbf{L 2}$ in $\mathrm{CHCl}_{3} / \mathrm{MeOH}(1 / 3)$. 
5. ${ }^{1} \mathrm{H}$ NMR, DEPTQ ${ }^{13} \mathrm{C}$ NMR, 2D COSY NMR, 2D NOESY NMR, 2D DOSY NMR, ESIMS and TWIM-MS spectra of Complexes HP1 and HP2

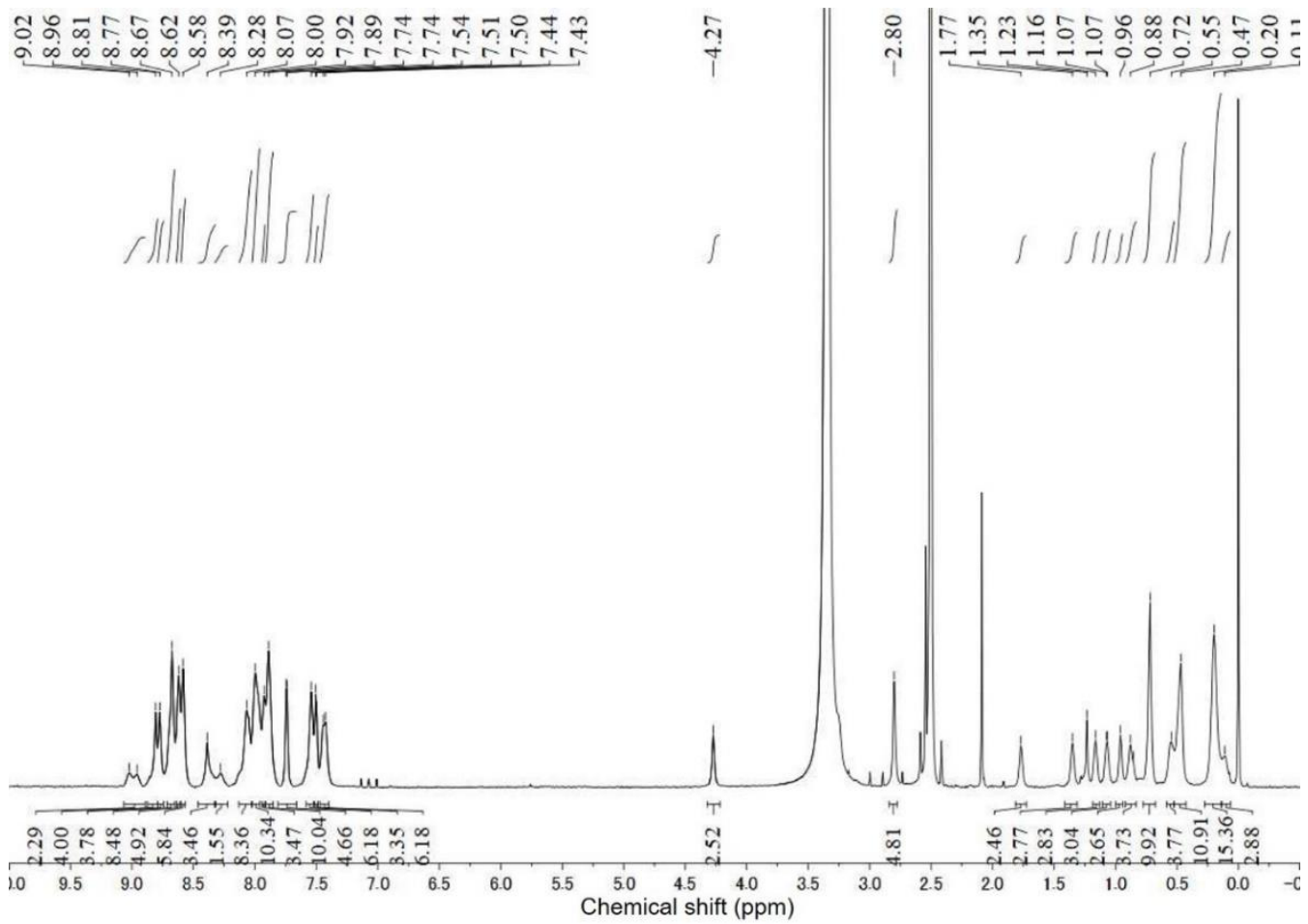

Figure S35: ${ }^{1} \mathrm{H}$ NMR $\left(600 \mathrm{MHz}, d_{6}-\mathrm{DMSO}, 300 \mathrm{~K}\right)$ spectrum of Complex HP1.

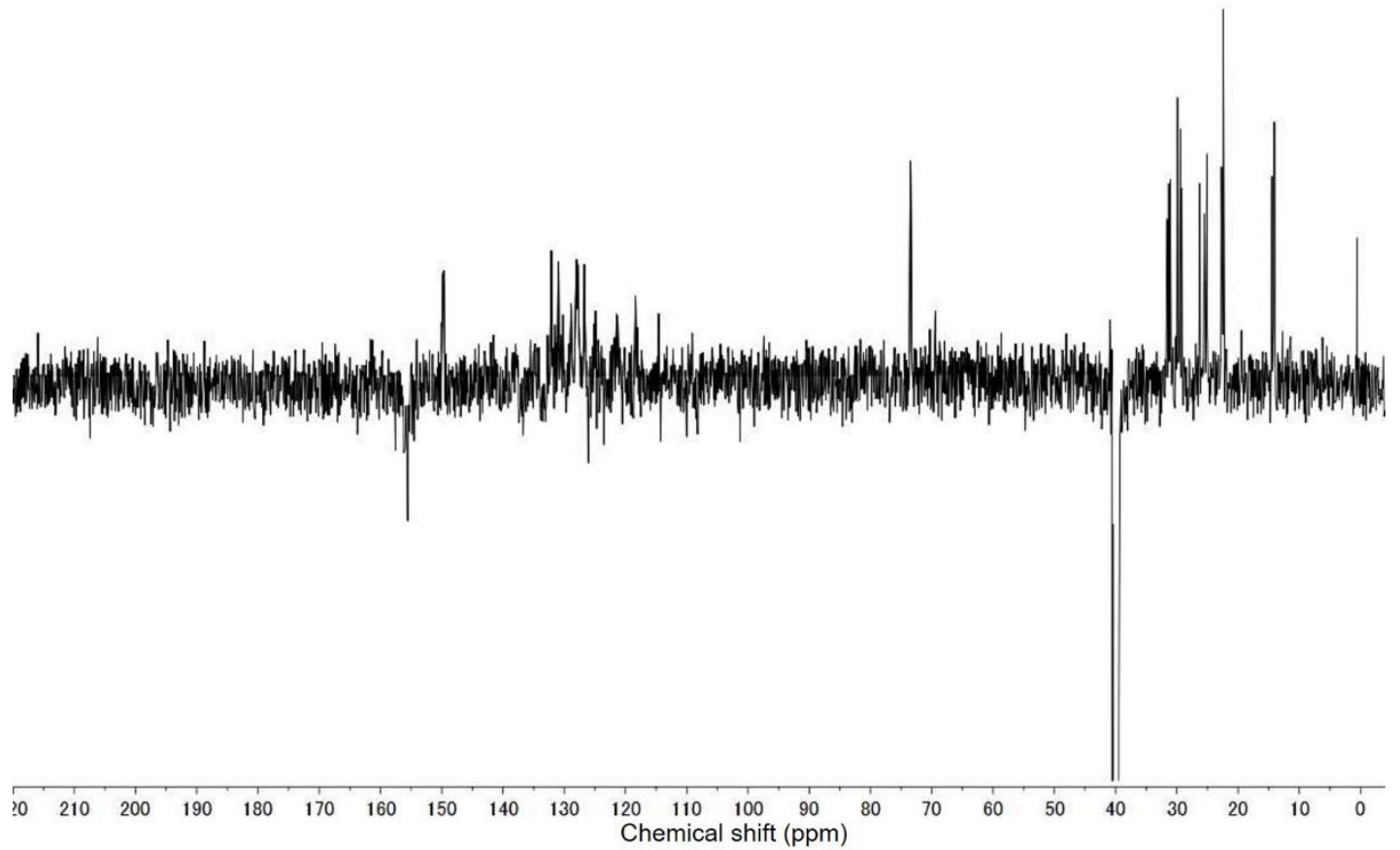

Figure S36: DEPTQ ${ }^{13} \mathrm{C}$ NMR $\left(125 \mathrm{MHz}, d_{6}\right.$-DMSO, $\left.300 \mathrm{~K}\right)$ spectrum of Complex HP1. 


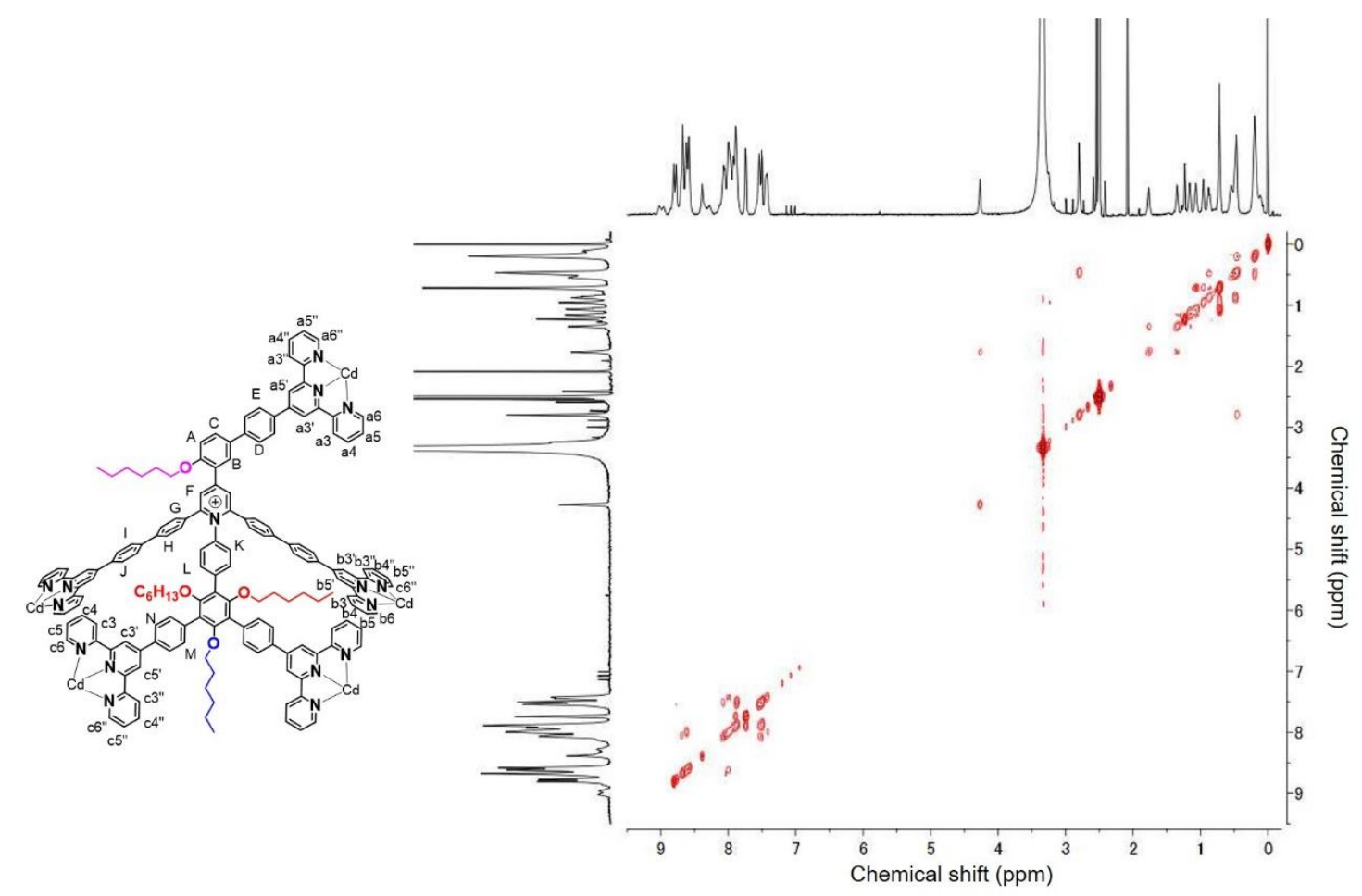

Figure S37: 2D COSY NMR (600 MHz, $d_{6}$-DMSO, $300 \mathrm{~K}$ ) spectrum of Complex HP1.

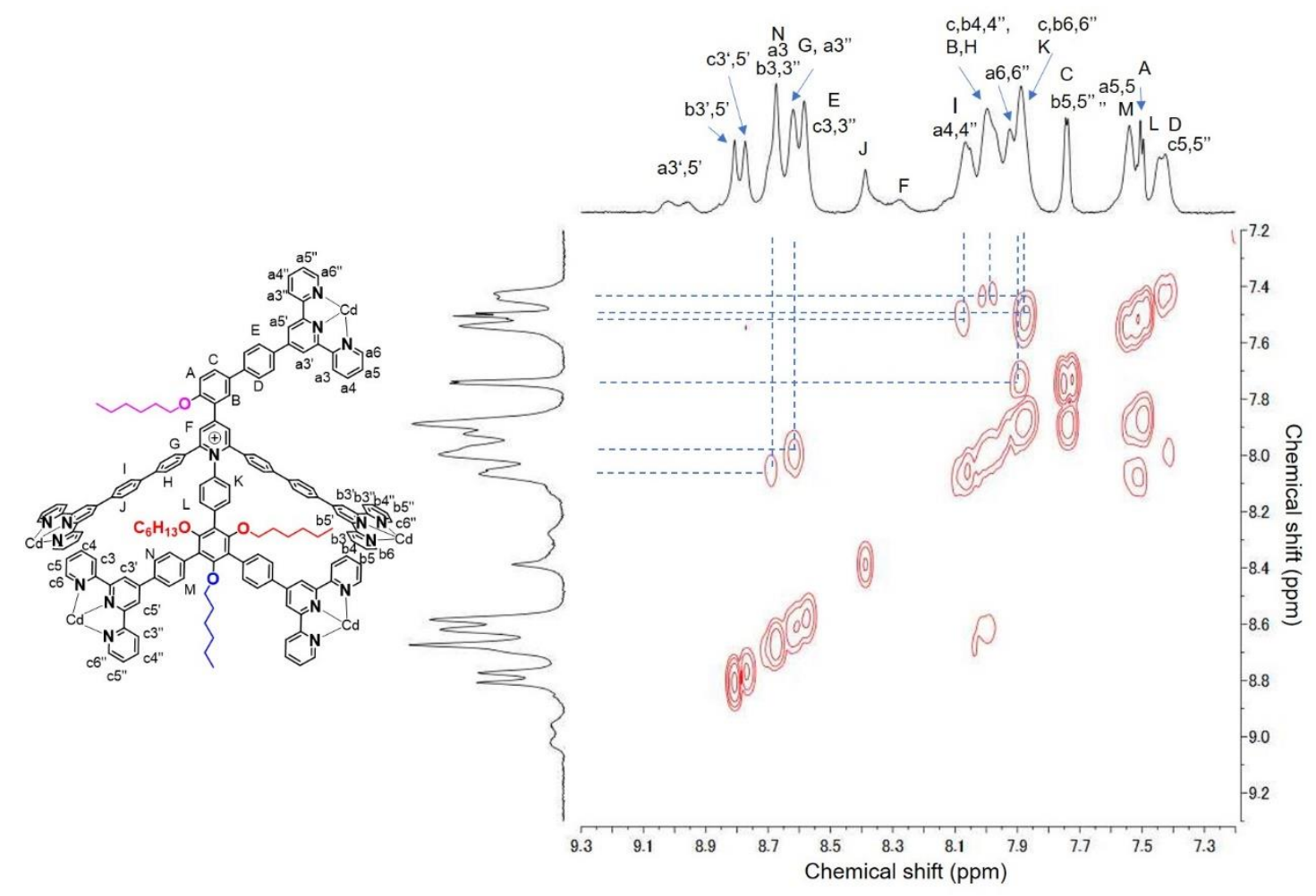

Figure S38: 2D COSY NMR (600 MHz, $d_{6}$-DMSO, $\left.300 \mathrm{~K}\right)$ spectrum of Complex HP1 (aromatic region). 


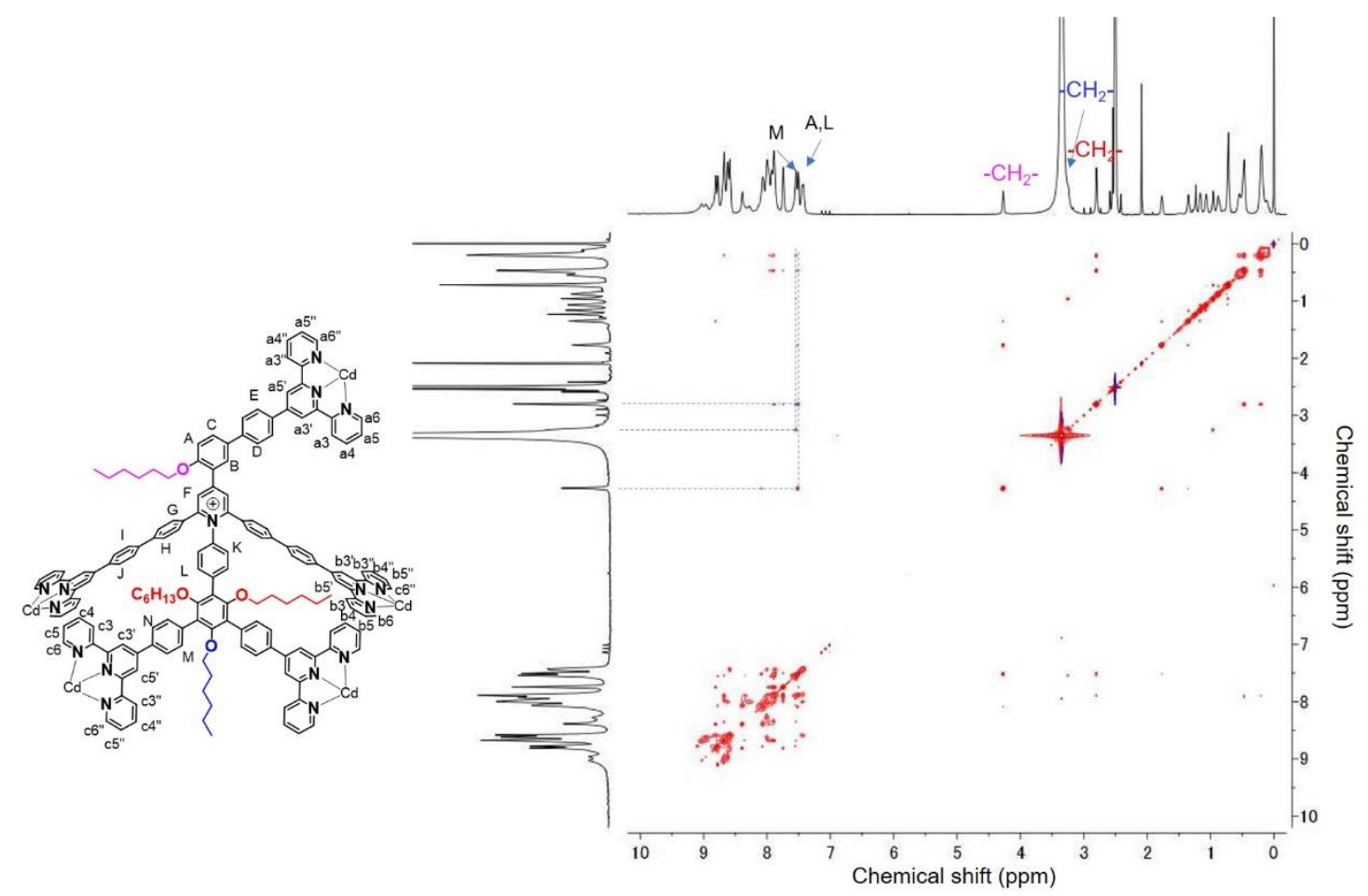

Figure S39: 2D NOESY NMR (600 MHz, $d_{6}$-DMSO, $300 \mathrm{~K}$ ) spectrum of Complex HP1.

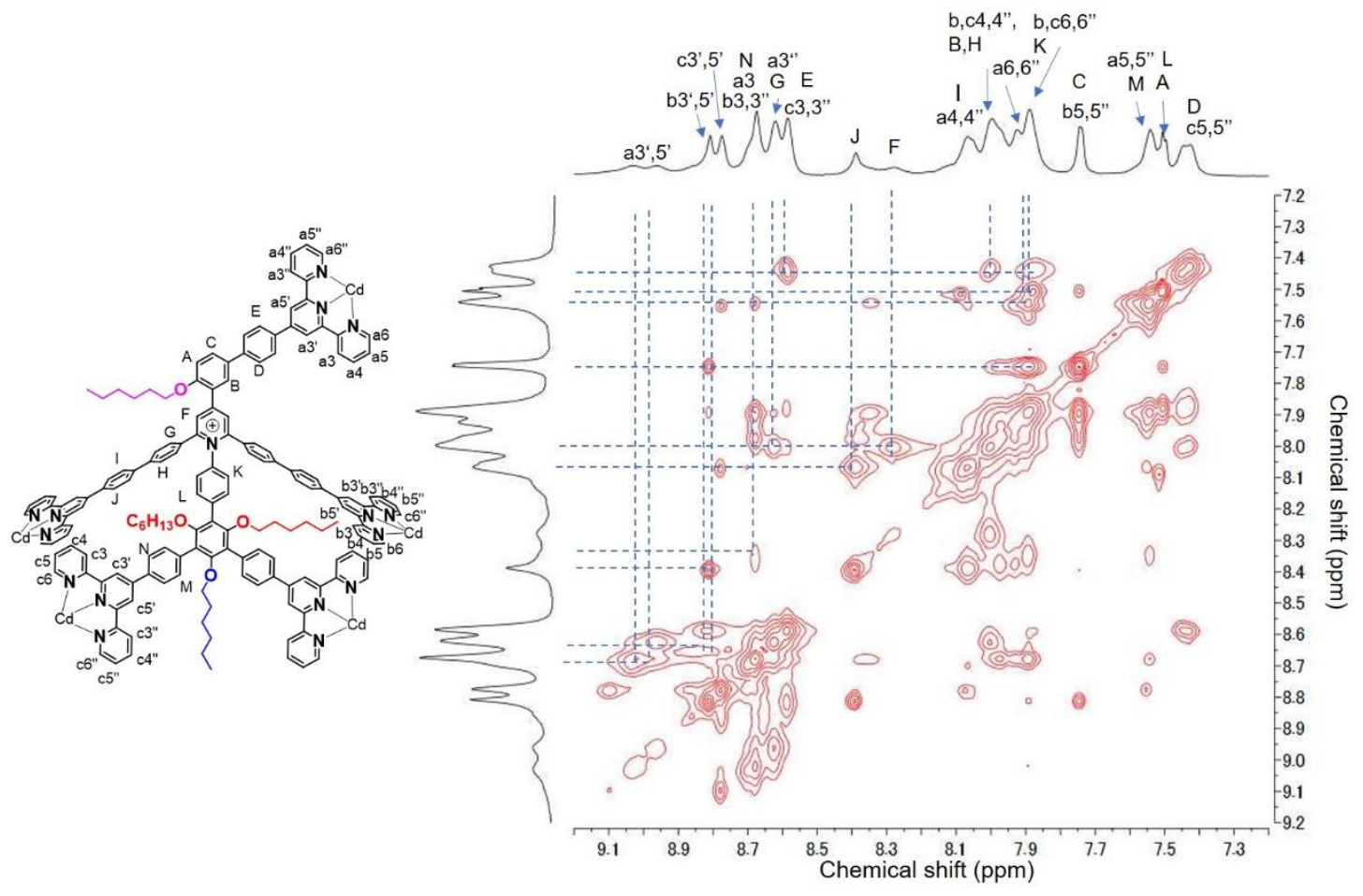

Figure S40: 2D NOESY NMR (600 MHz, $d_{6}$-DMSO, $\left.300 \mathrm{~K}\right)$ spectrum of Complex HP1 (aromatic region). 


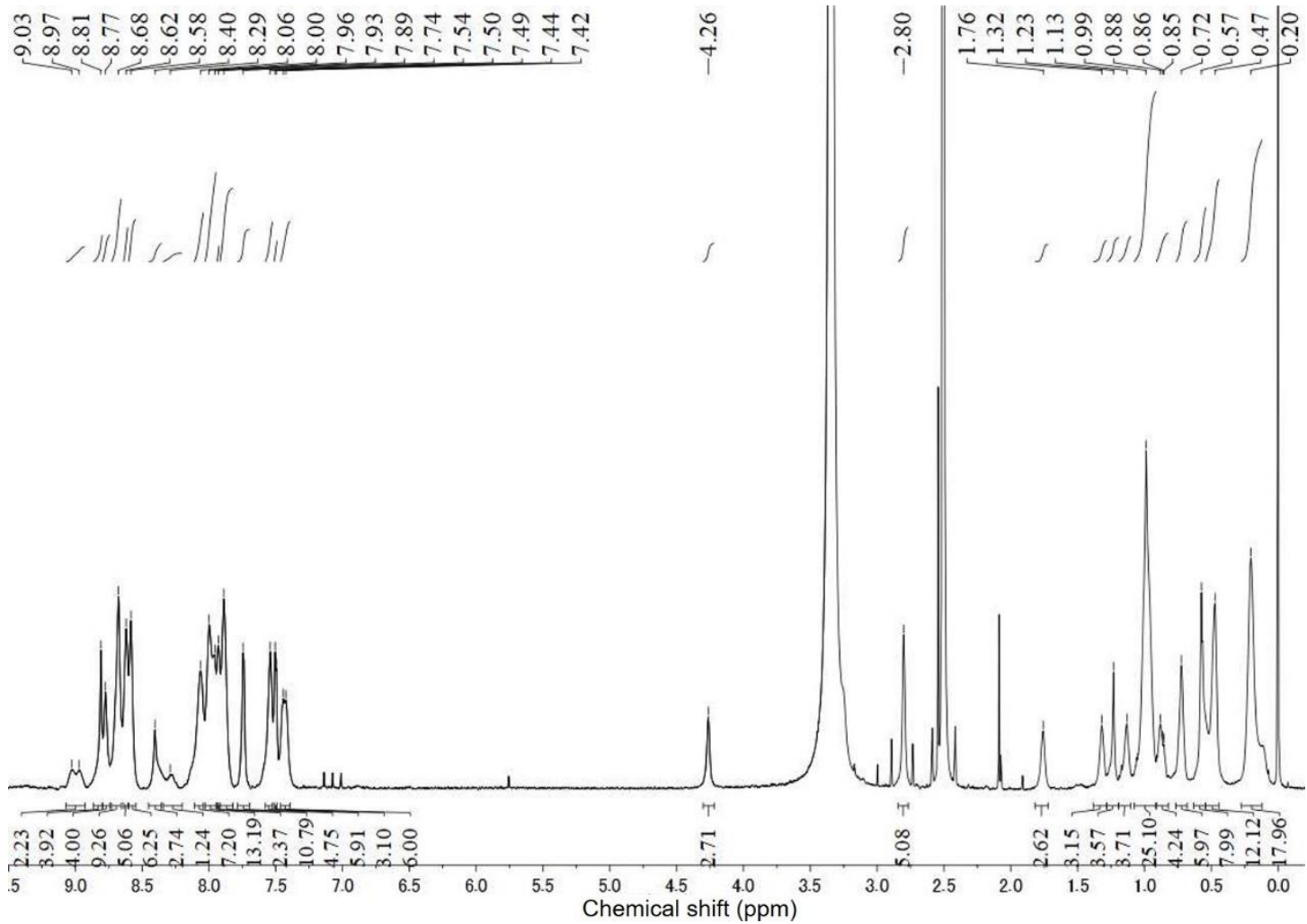

Figure S41: ${ }^{1} \mathrm{H}$ NMR $\left(600 \mathrm{MHz}, d_{6}\right.$-DMSO, $\left.300 \mathrm{~K}\right)$ spectrum of Complex HP2.

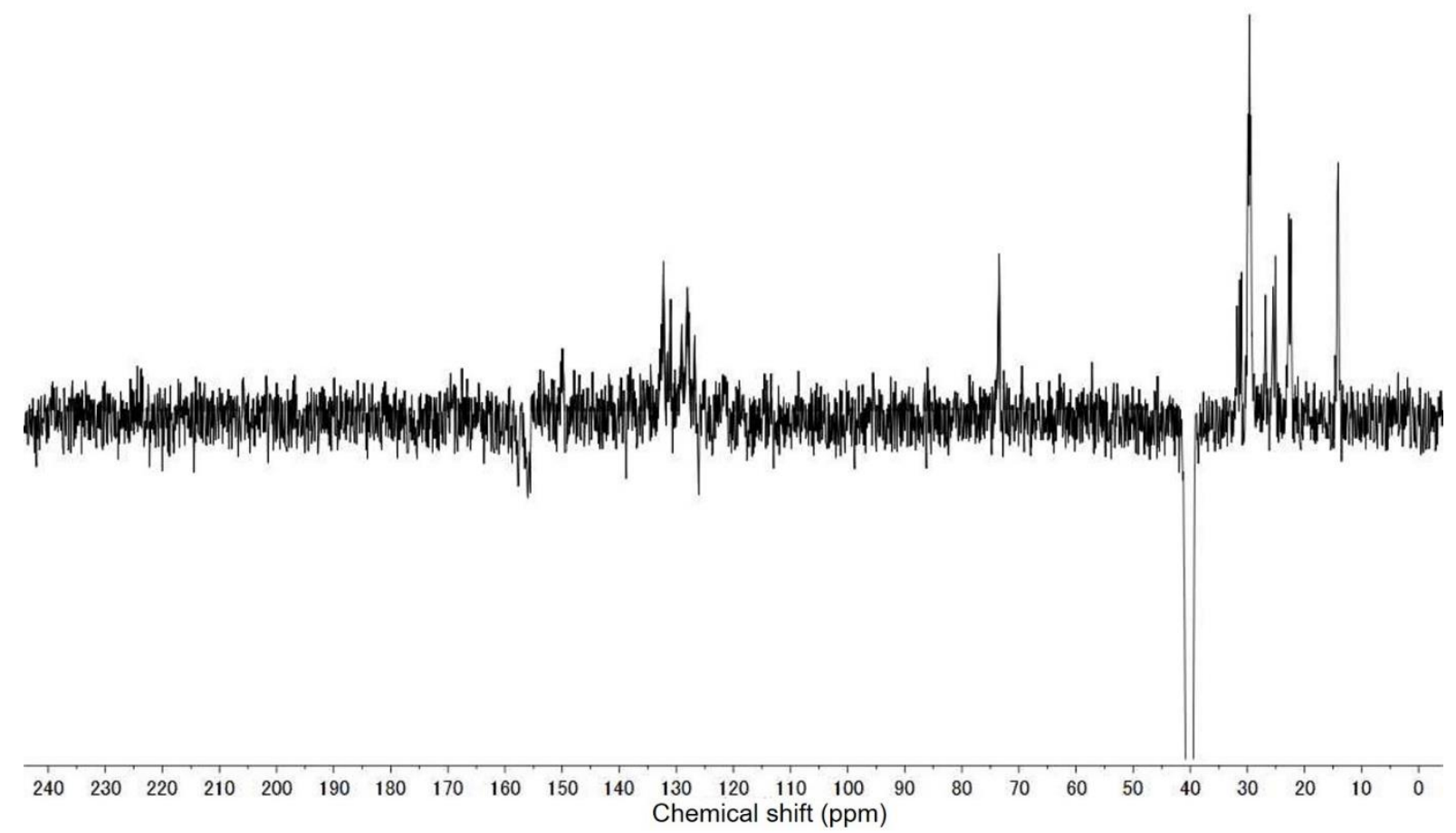

Figure S42: DEPTQ ${ }^{13} \mathrm{C}$ NMR $\left(125 \mathrm{MHz}, d_{6}\right.$-DMSO, $\left.300 \mathrm{~K}\right)$ spectrum of Complex HP2. 


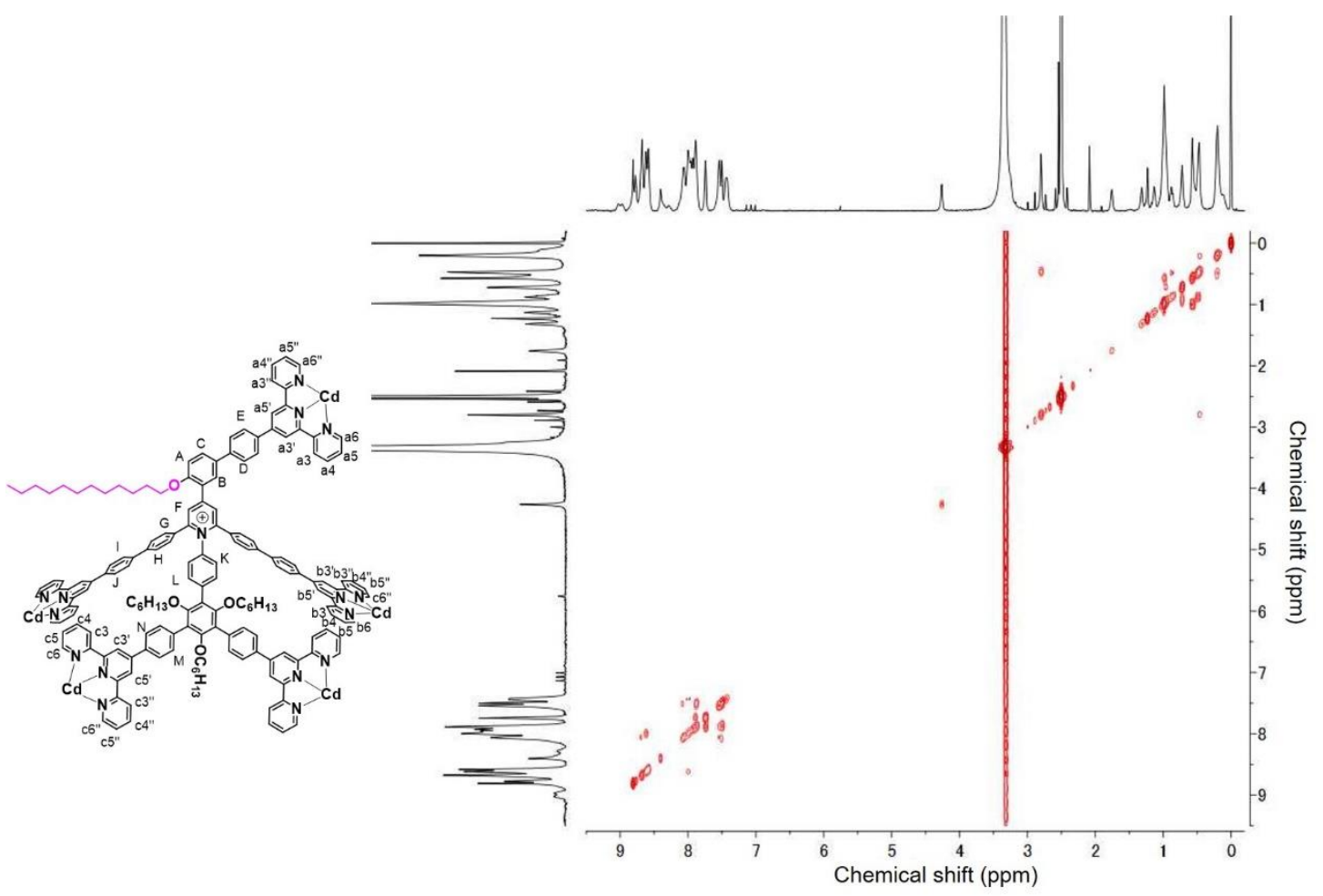

Figure S43: 2D COSY NMR (600 MHz, $d_{6}$-DMSO, $300 \mathrm{~K}$ ) spectrum of Complex HP2.

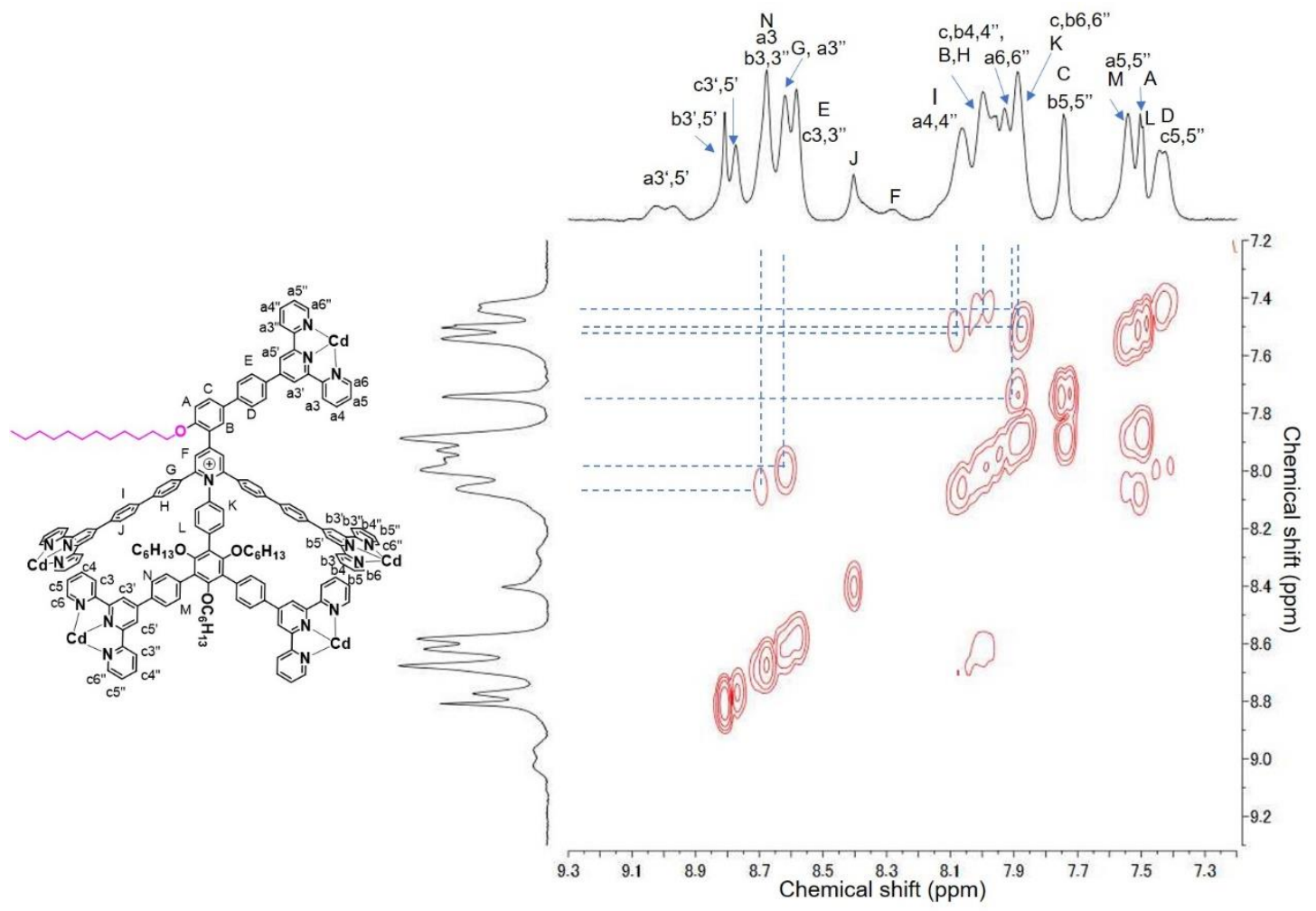

Figure S44:2D COSY NMR (600 MHz, $d_{6}$-DMSO, $\left.300 \mathrm{~K}\right)$ spectrum of Complex HP2 (aromatic region). 


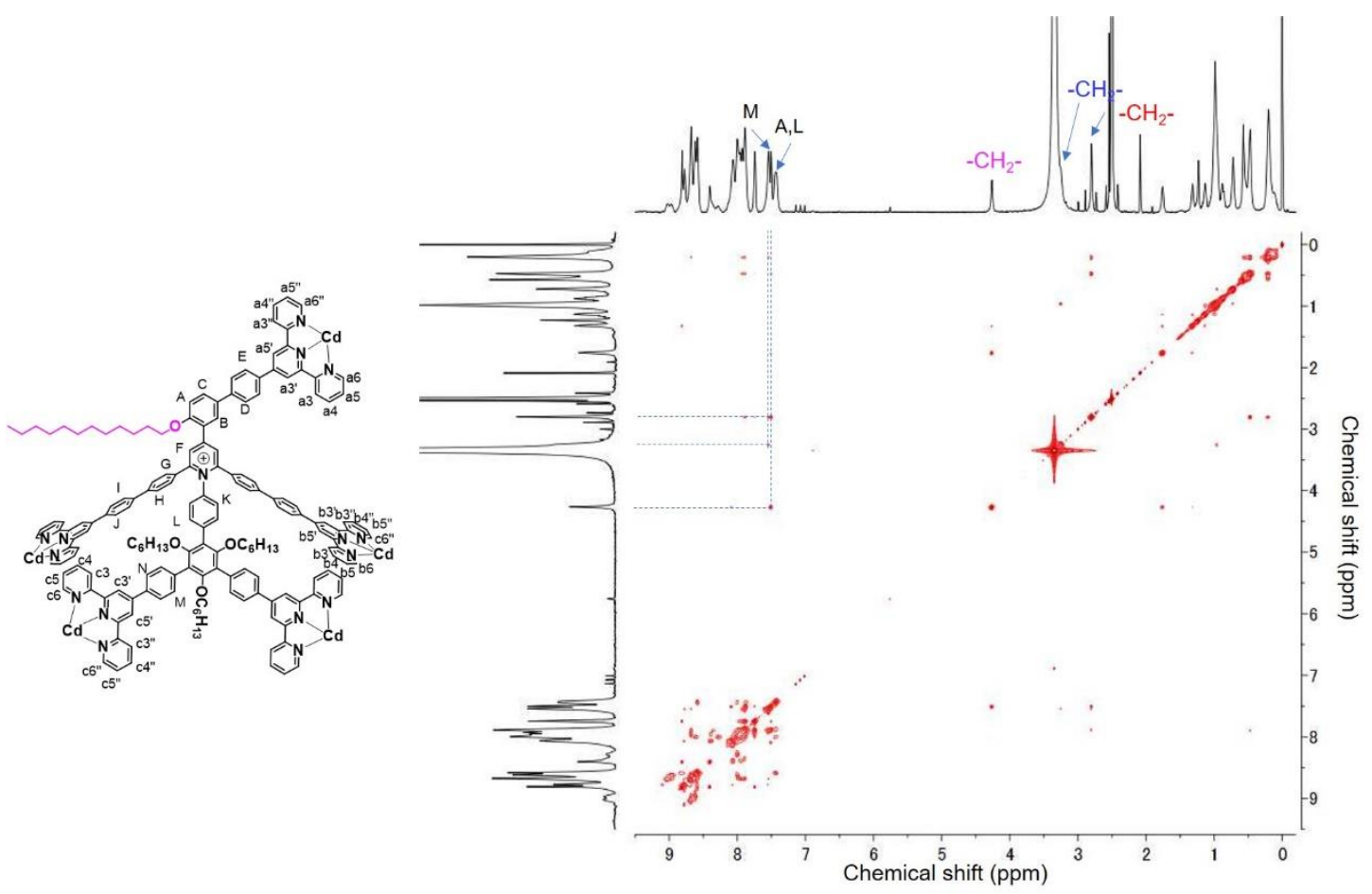

Figure S45: 2D NOESY NMR (600 MHz, $d_{6}$-DMSO, $300 \mathrm{~K}$ ) spectrum of Complex HP2.

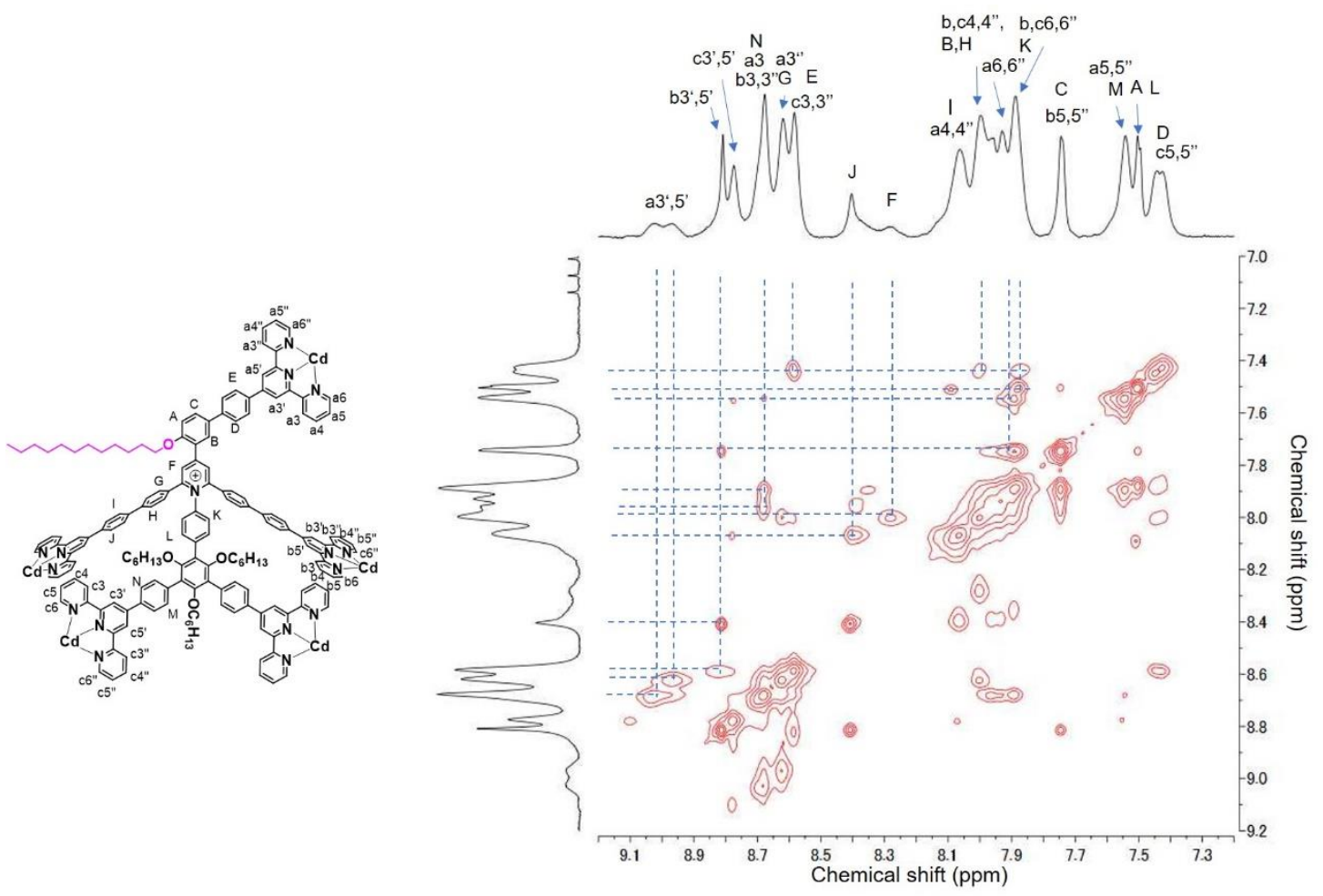

Figure S46: 2D NOESY NMR (600 MHz, $d_{6}$-DMSO, $\left.300 \mathrm{~K}\right)$ spectrum of Complex HP2 (aromatic region). 


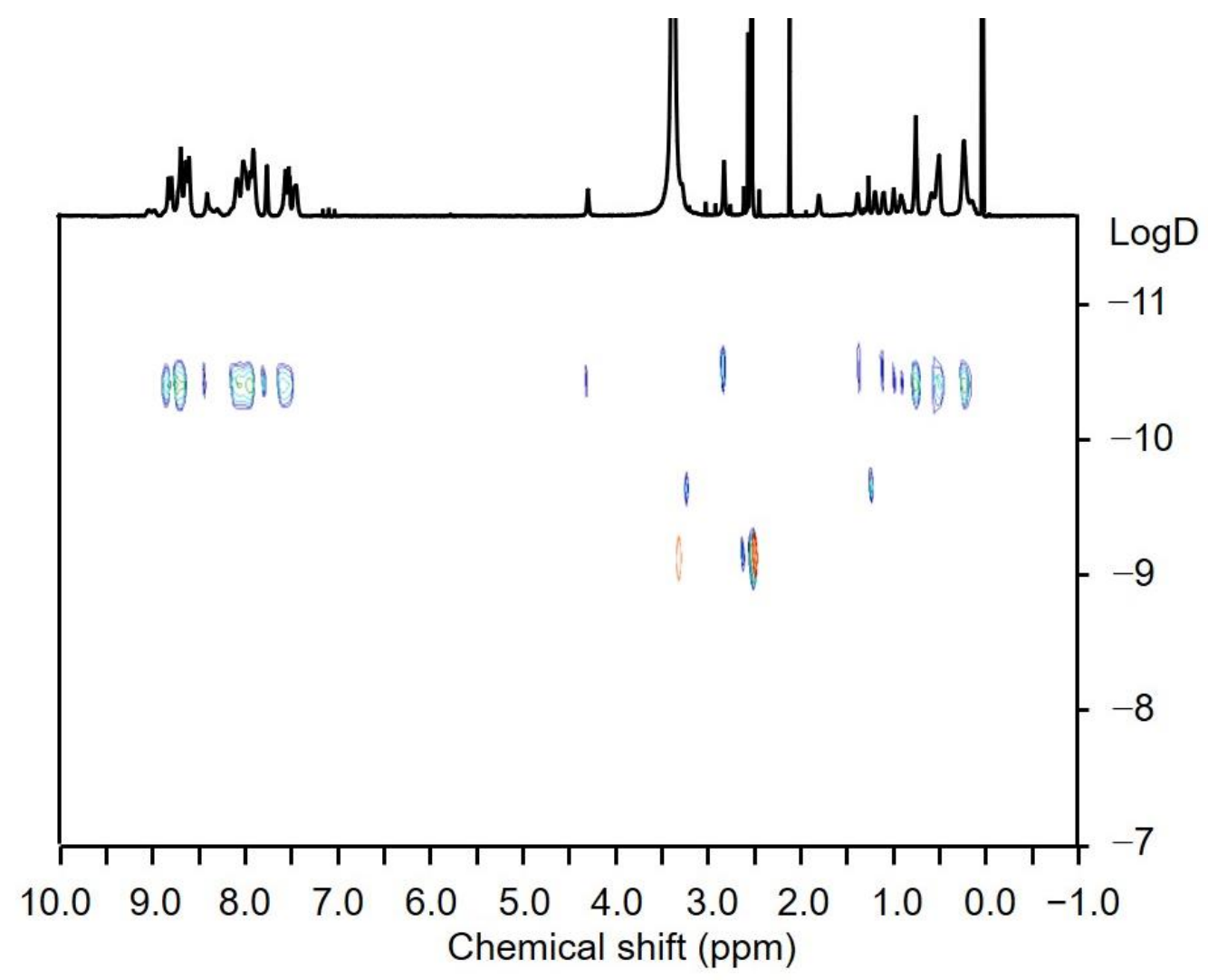

Figure S47: 2D DOSY ( $600 \mathrm{MHz}, d_{6}$-DMSO, $\left.300 \mathrm{~K}\right)$ spectrum of HP1 with $\log \mathrm{D} \approx-10.5$.

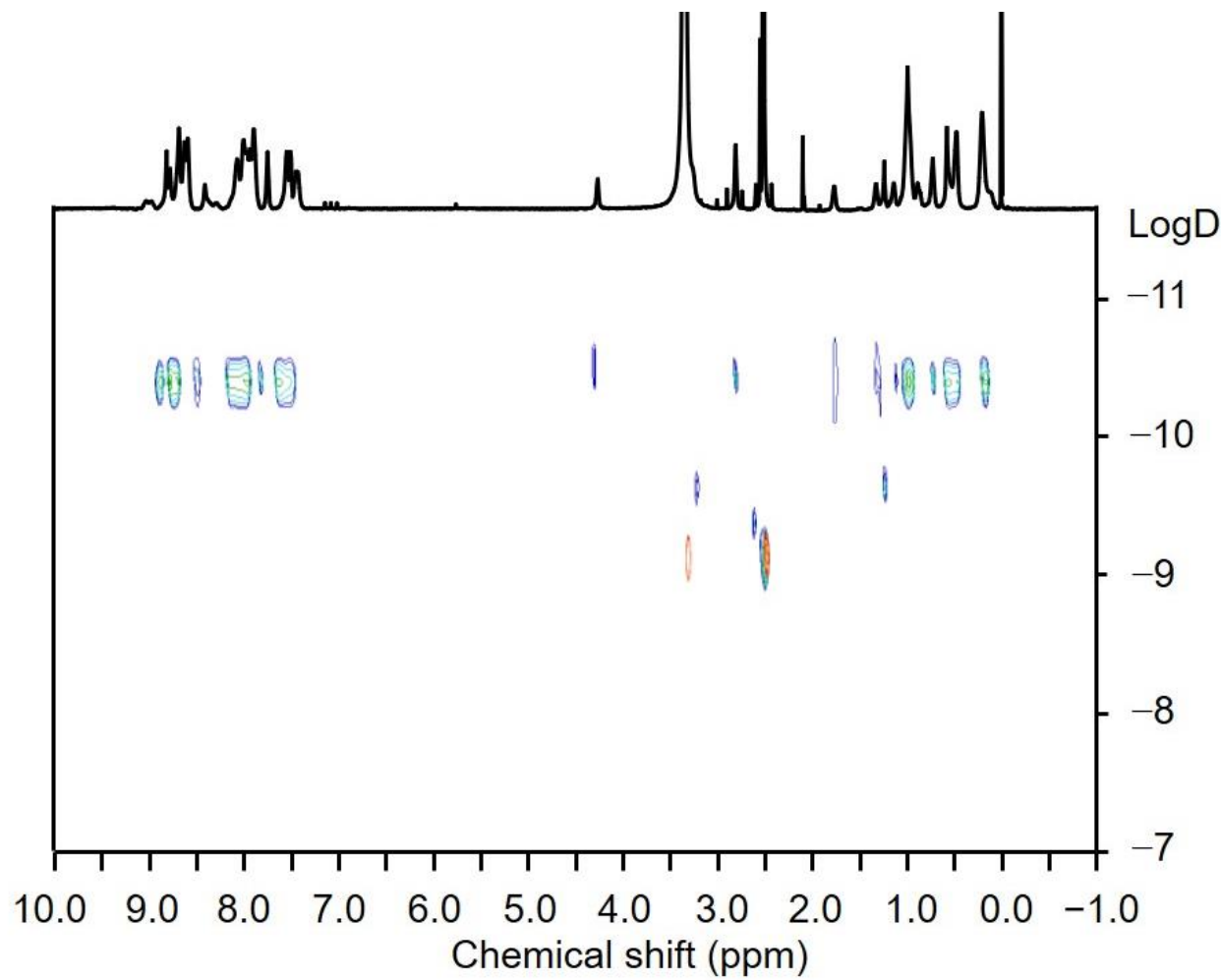

Figure S48: 2D DOSY (600 MHz, $d_{6}$-DMSO, $\left.300 \mathrm{~K}\right)$ spectrum of HP2 with $\log \mathrm{D} \approx-10.5$. 


\section{TEM, AFM and STM images}
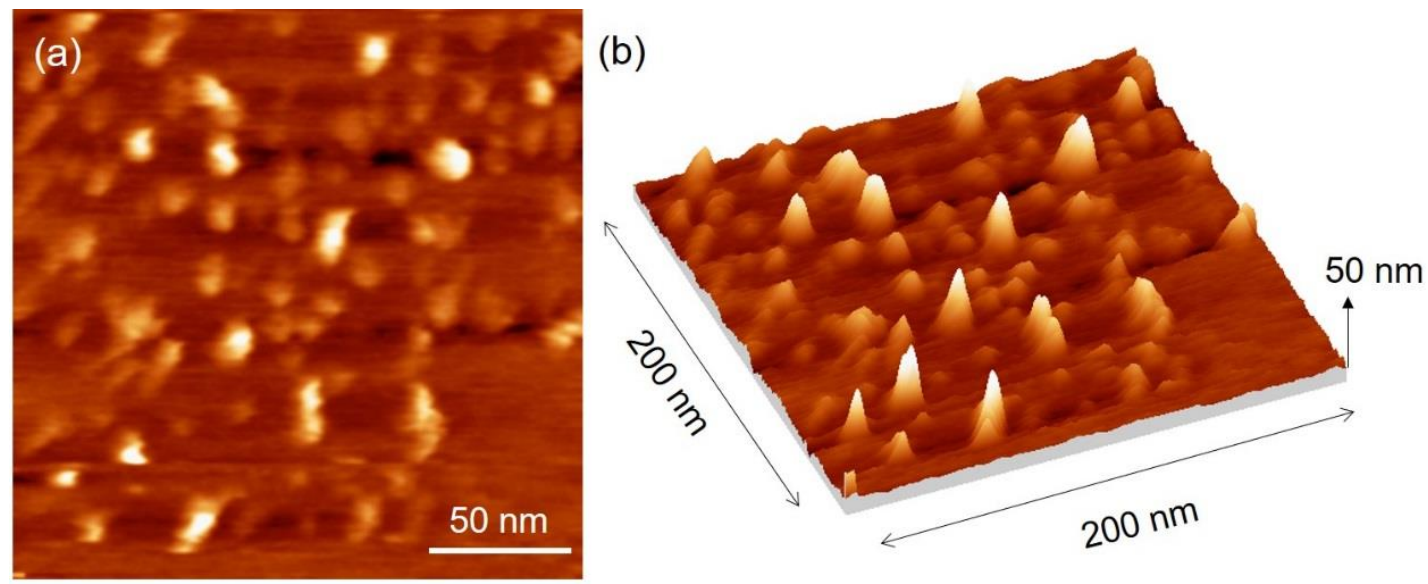

Figure S49: Large area of (a) AFM image and (b) 3D AFM image.
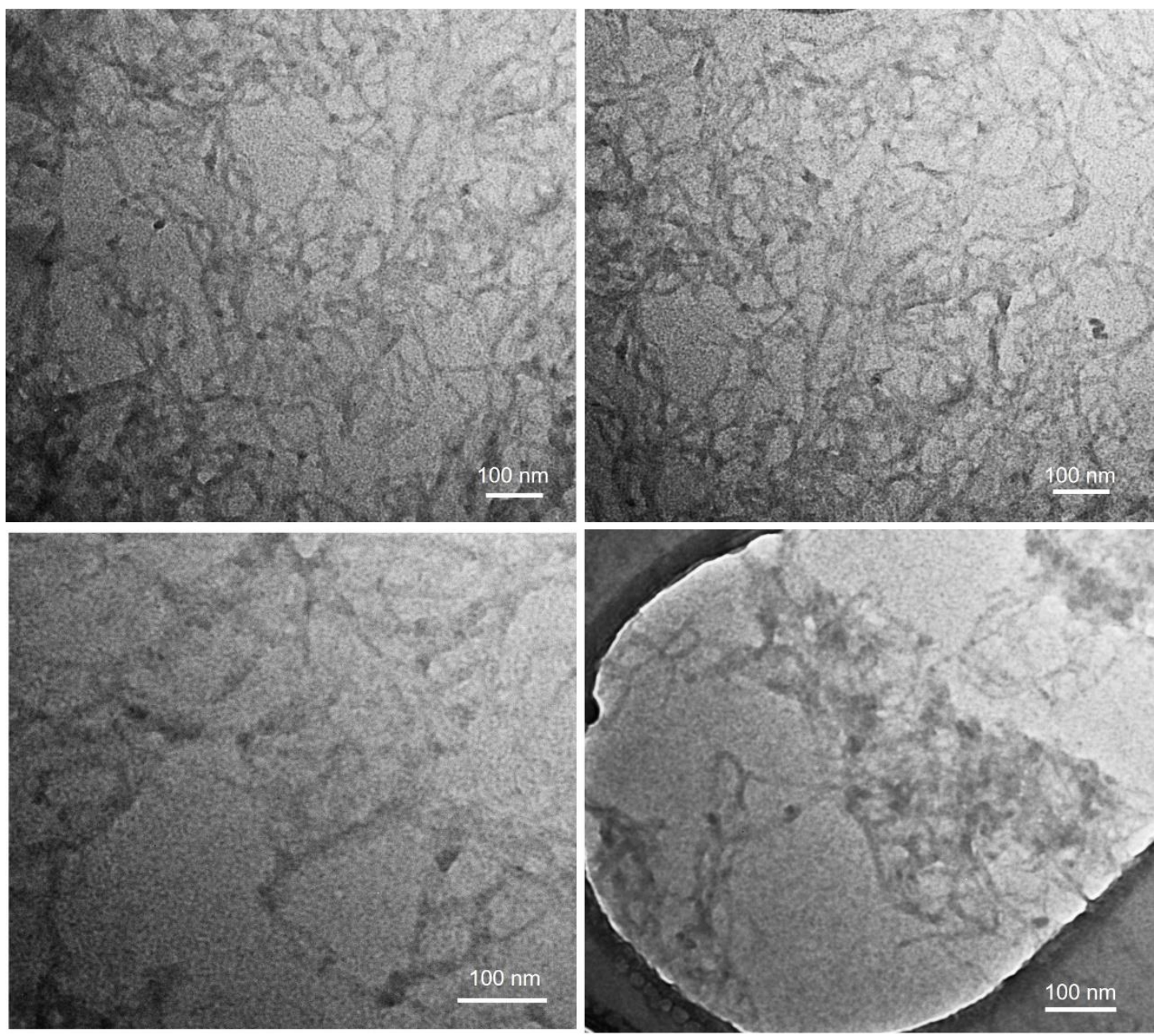

Figure S50: TEM images of nanostructures formed by HP1 in DMF ( $5 \mathrm{mg} / \mathrm{mL}$ ) by slowly vaporing ethyl acetate. 


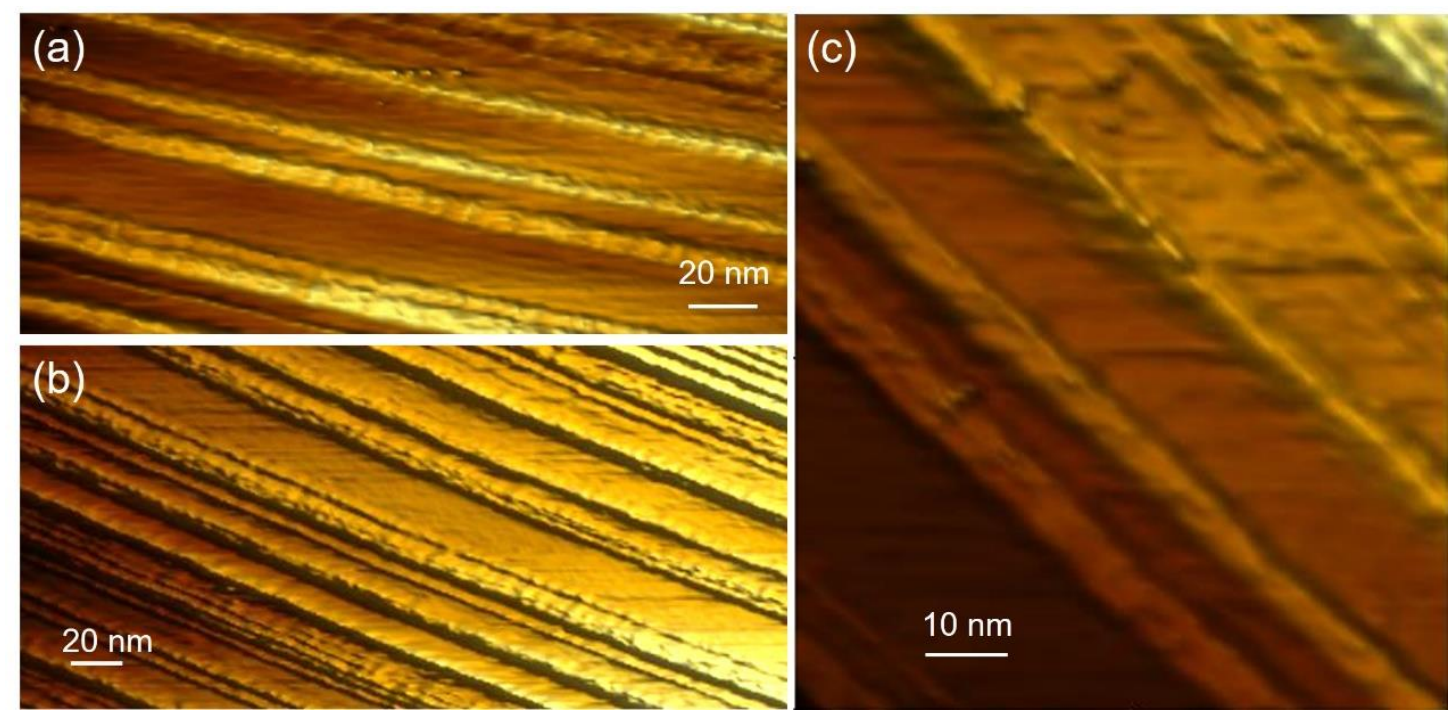

Figure S51: More STM images of packed HP1 tubular-like nanostructures on HOPG surfaces. 


\section{Mechanism study of self-assembly of HP1}

Sample preparation: To a solution of ligand L1 $(10.0 \mathrm{mg}, 4.03 \mu \mathrm{mol})$ in DMSO $(4.5 \mathrm{~mL})$, a solution of $\mathrm{Cd}\left(\mathrm{NO}_{3}\right)_{2} \cdot 4 \mathrm{H}_{2} \mathrm{O}(3.1 \mathrm{mg}, 10 \mu \mathrm{mol})$ in DMSO $(0.5 \mathrm{~mL})$ was added, and then the mixture was kept at $50{ }^{\circ} \mathrm{C}$ for $3 \mathrm{~h}$. After cooling to room temperature, the assembly solution was added to $10 \mathrm{~mL} \mathrm{CH}_{3} \mathrm{OH}$ solution of $\mathrm{NH}_{4} \mathrm{PF}_{6}(80 \mathrm{mg})$, and yellow precipitate was formed. The precipitate was collected by centrifugation and washed by fresh $\mathrm{CH}_{3} \mathrm{OH}$ and water repeatedly. The collected gel like precipitate was directly used for ESI-MS and TWIM-MS characterization.
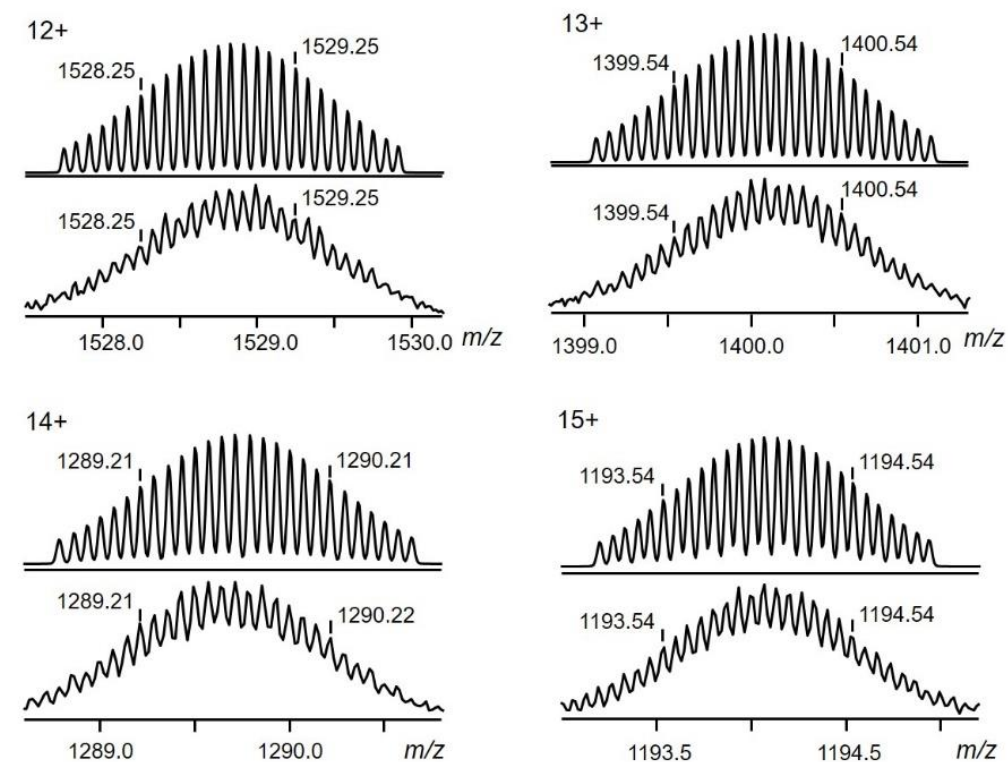

$15+$
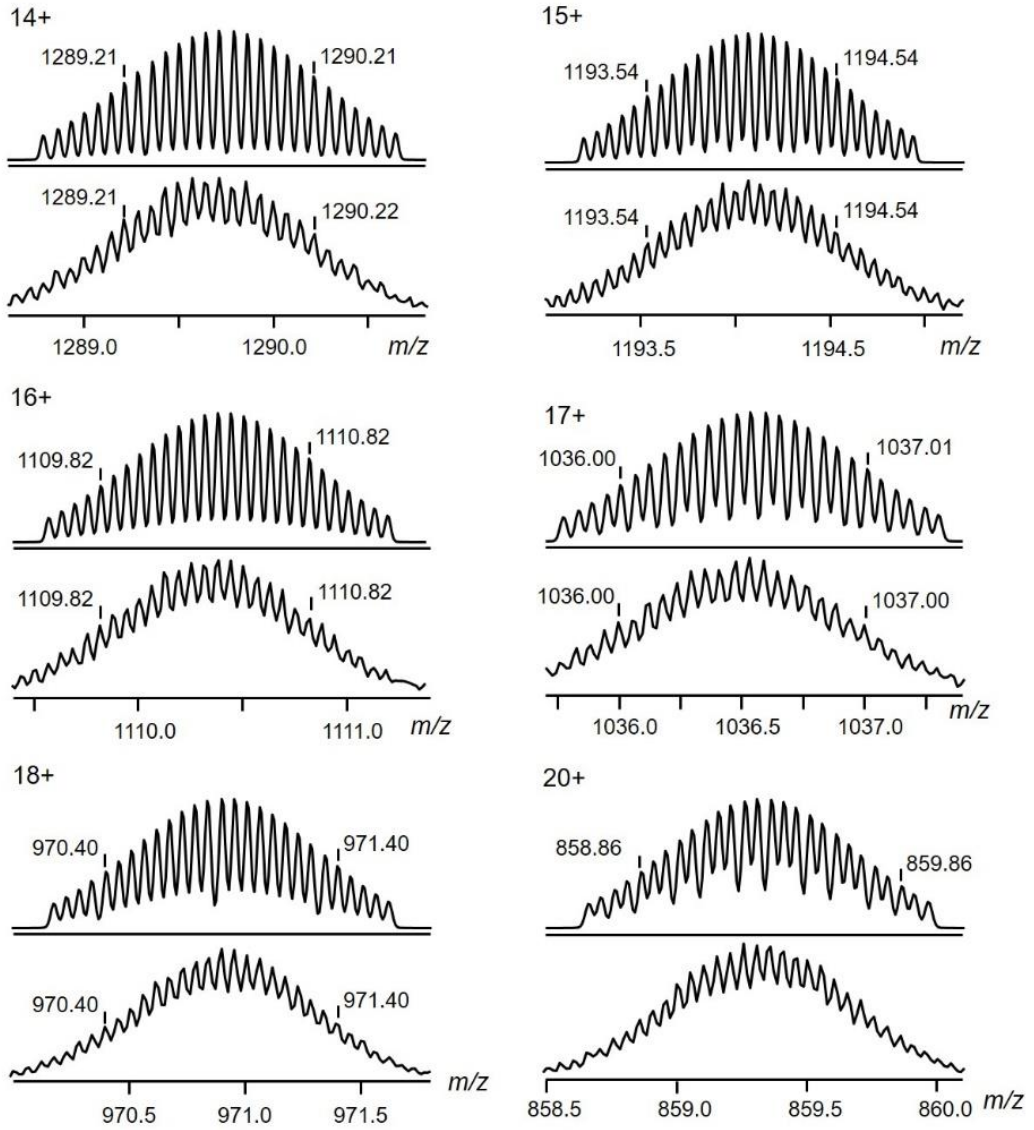

Figure S52: Experimental (bottom) and calculated (top) isotope patterns for different charge states observed from intermediate $\left[(\mathbf{L 1})_{6} \mathrm{Cd}_{12}\left(\mathrm{PF}_{6}\right)_{30}\right]$. 
$\left[(\mathbf{L} 1)_{6} \mathrm{Cd}_{12}\left(\mathrm{PF}_{6}\right)_{13}\right]^{17+}$

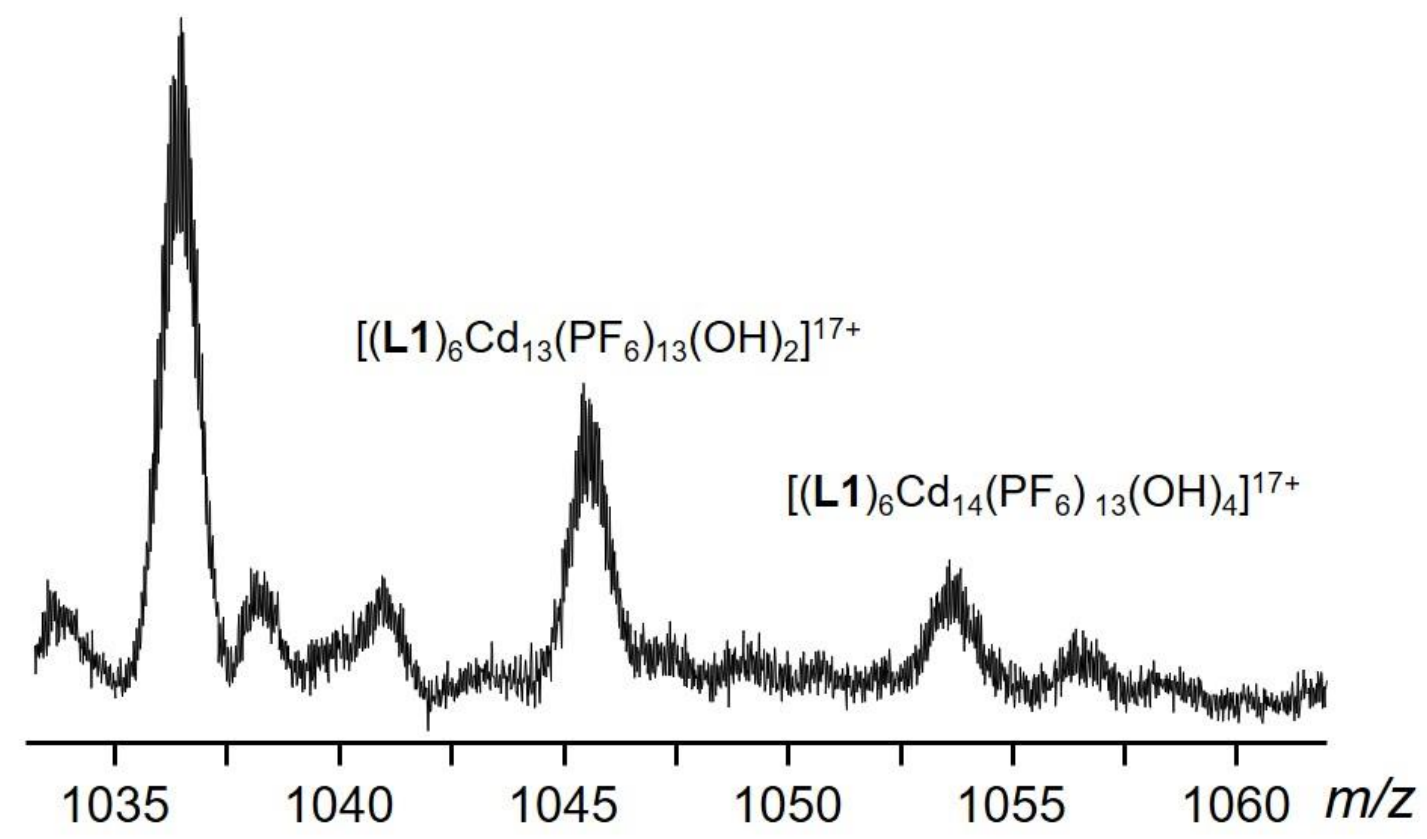

Figure S53: Expanded ESI-MS spectrum of the self-assembly of L1 with Cd(II) under a milder condition (full spectrum shown in Fig. 6). Peaks were assigned to three different intermediates $\left[(\mathbf{L 1})_{6} \mathrm{Cd}_{12}\left(\mathrm{PF}_{6}\right)_{30}\right],\left[(\mathbf{L 1})_{6} \mathrm{Cd}_{13}\left(\mathrm{PF}_{6}\right)_{30}(\mathrm{OH})_{2}\right]$ and $\left[(\mathbf{L 1})_{6} \mathrm{Cd}_{14}\left(\mathrm{PF}_{6}\right)_{30}(\mathrm{OH})_{4}\right]$ were observed.
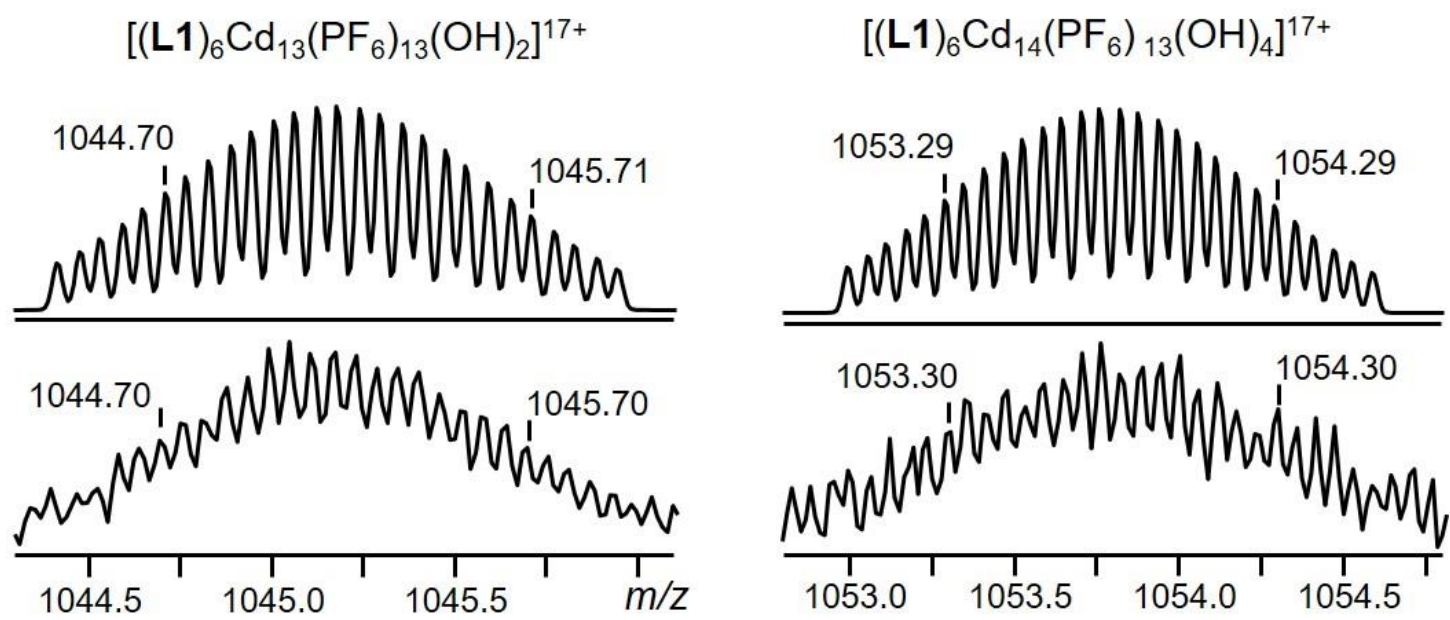

Figure S54: Experimental (bottom) and calculated (top) isotope patterns of intermediates $\left[(\mathbf{L 1})_{6} \mathrm{Cd}_{13}\left(\mathrm{PF}_{6}\right)_{13}(\mathrm{OH})_{2}\right]^{17+}$ and $\left[(\mathbf{L 1})_{6} \mathrm{Cd}_{14}\left(\mathrm{PF}_{6}\right)_{13}(\mathrm{OH})_{4}\right]^{17+}$. 


\section{UV-Vis spectra}
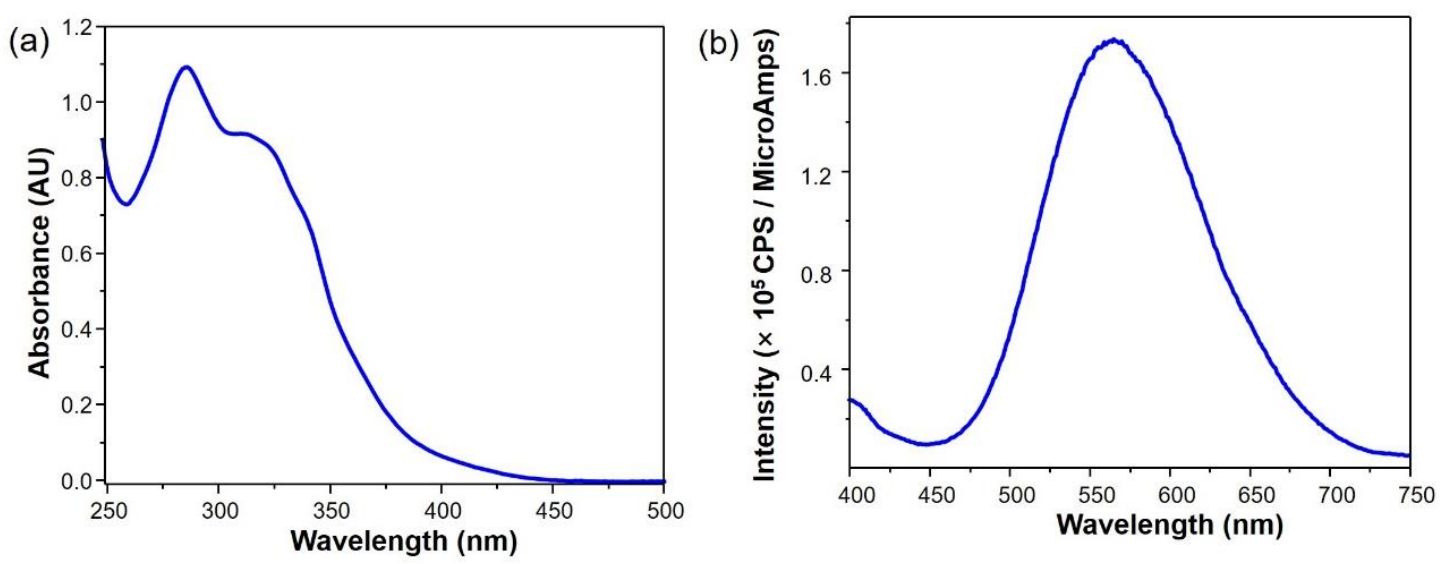

Figure S55: (a) Absorption spectrum and (b) emission spectrum of $\mathbf{H P 1}$ in $\mathrm{DMSO} / \mathrm{CH}_{3} \mathrm{CN}$ $(1 / 9)$ mixtures $\left(\lambda_{\mathrm{ex}}=320 \mathrm{~nm}, \mathrm{c}=1 \mu \mathrm{mol} / \mathrm{L}\right)$.
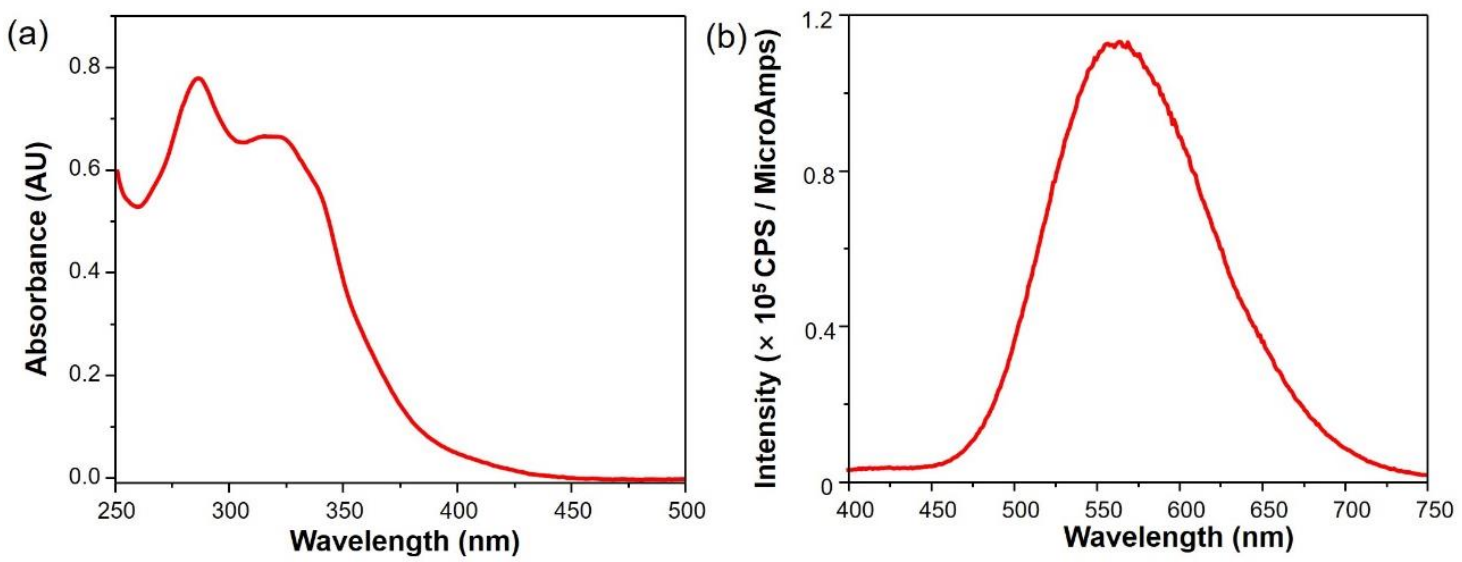

Figure S56: (a) Absorption spectrum and (b) emission spectrum of HP2 in $\mathrm{DMSO} / \mathrm{CH}_{3} \mathrm{CN}$ $(1 / 9)$ mixtures $\left(\lambda_{\mathrm{ex}}=320 \mathrm{~nm}, \mathrm{c}=1 \mu \mathrm{mol} / \mathrm{L}\right)$. 


\section{Fitting procedure to derive size parameter of $\mathrm{HP1}$ from $\log \mathrm{D}^{8}$}

\begin{tabular}{|c|c|c|c|c|c|c|}
\hline \multicolumn{7}{|l|}{ HP1 } \\
\hline$d^{6} 6-\mathrm{DMSO}$ & & $\mathrm{kb}$ & 1. $38 \mathrm{E}-23$ & & & \\
\hline \multicolumn{7}{|c|}{ Cylindrical model } \\
\hline $\log (D)$ & -10.49 & D & 3. $2 \mathrm{E}-11$ & & & \\
\hline$T(\mathrm{~K})$ & 298 & & & & & \\
\hline$\eta(\mathrm{Pa} \mathrm{s})$ & 0.00224 & dmso & & & & \\
\hline $\operatorname{rh}(\mathrm{sp})=$ & $\mathrm{sbT} / 6 \pi \mathrm{D} \eta$ & 3. $04 \mathrm{E}-09$ & $\mathrm{~m}$ & & & \\
\hline $\mathrm{L}$ & OD & $\operatorname{rh}(\mathrm{sp})$ & $\operatorname{rh}(o b)$ & fs & $r(\mathrm{cal})$ & $r(\mathrm{ob}) / \mathrm{rh}(\mathrm{cal})$ \\
\hline $2.50 \mathrm{E}-09$ & $7.00 \mathrm{E}-09$ & $3.04 \mathrm{E}-09$ & 2. $84 \mathrm{E}-09$ & 1. $07 \mathrm{E}+00$ & $2.84 \mathrm{E}-09$ & $9.99 \mathrm{E}-01$ \\
\hline
\end{tabular}

Figure S57. Screenshot of Microsoft Excel spreadsheet used to fit HP1 in $d_{6}$-DMSO using cylindrical model.

Spreadsheet details

- For sphere model, according to Stocks-Einstein equation, the hydrodynamic radius $\left(r_{\mathrm{h}}(\mathrm{sp})\right)$

of particles has the relationship with diffusion coefficients $(D)$ :

$$
D=\frac{k_{B} T}{6 \pi \eta r_{h}(s p)}
$$

For non-sphere situation, the relationship between $D$ and $r_{\mathrm{h}}$ is modified:

$$
D=\frac{k_{B} T}{c f_{S} \pi \eta r_{h}(o b)}
$$

Where:

$$
c=\frac{6}{1+0.695\left(\frac{r_{v d W}}{r_{h}}\right)^{2.234}}
$$

$r_{\mathrm{vdW}}$ is the van der Waals radii of the solvent, $d_{6}$-DMSO, which is far less than $r_{\mathrm{h}}$ of HP1.

As a result, $c \approx 6$. " $f_{\mathrm{s}}$ " is the frictional coefficient, and it is derived from the height (L) and the diameter (OD) of the cylinder for the cylindrical model with the formula:

$$
\begin{aligned}
f_{S}=1.0304 & +0.0193\left[\ln \left(\frac{L}{O D}\right)\right]+0.06229\left[\ln \left(\frac{L}{O D}\right)\right]^{2}+0.00476\left[\ln \left(\frac{L}{O D}\right)\right]^{3} \\
& +0.00166\left[\ln \left(\frac{L}{O D}\right)\right]^{4}+2.66 \times 10^{-6}\left[\ln \left(\frac{L}{O D}\right)\right]^{7}
\end{aligned}
$$

As a result:

$$
r_{h}(o b)=\frac{r_{h}(s p)}{f_{s}}
$$

- Values in the blue boxes (L and OD in meter) are the only ones iteratively changed 
- " $k$ " " is the Boltzmann constant, " $T$ " is the experimental temperature $(300 \mathrm{~K})$, and " $\eta "$ is the viscosity of $d_{6}$-DMSO $(0.00224 \mathrm{~Pa} \mathrm{~s})$.

- " $r(\mathrm{cal}) "$ is the radius of a sphere with an equivalent volume as the spheroid generated by L and OD; since spheroid volume $(\mathrm{V})$ has the relationship with $\mathrm{L}$ and $\mathrm{OD}: \mathrm{V}=\frac{1}{4} \pi \mathrm{L}(\mathrm{OD})^{2}$, and $r(\mathrm{cal})=\sqrt[3]{\frac{3 V}{4 \pi}}, r(\mathrm{cal})$ can be calculated by the formula: $r(\mathrm{cal})=\sqrt[3]{\frac{3}{16} \mathrm{~L}(\mathrm{OD})^{2}}$

- " $r \mathrm{~h}(\mathrm{ob}) / r(\mathrm{cal}) "$ is the ratio of the hydrodynamic radius generated from the cylindrical model and the equivalent radius generated by the volume of the spheroid; this ratio was used to guide the adjustment of L and OD until the volume generated by them matches the volume generated by $r_{\mathrm{h}}(\mathrm{ob})\left(r_{\mathrm{h}}(\mathrm{ob}) / r(\mathrm{cal})=1\right)$

\section{References}

1. Wang, M.; Wang, K.; Wang, C.; Huang, M.; Hao, X.-Q.; Shen, M.-Z.; Shi, G.-Q.; Zhang, Z.; Song, B.; Cisneros, A.; Song, M.-P.; Xu, B.; Li, X., Self-Assembly of Concentric Hexagons and Hierarchical SelfAssembly of Supramolecular Metal-Organic Nanoribbons at the Solid/Liquid Interface. J. Am. Chem. Soc. 2016, 138 (29), 9258-9268.

2. (a) Casewit, C. J.; Colwell, K. S.; Rappe, A. K., Application of a Universal Force Field to Organic Molecules. J. Am. Chem. Soc. 1992, 114 (25), 10035-10046; (b) Casewit, C. J.; Colwell, K. S.; Rappe, A. K., Application of a Universal Force Field to Main Group Compounds. J. Am. Chem. Soc. 1992, 114 (25), 1004610053; (c) Rappe, A. K.; Casewit, C. J.; Colwell, K. S.; Goddard, W. A.; Skiff, W. M., UFF, a Full Periodic Table Force Field for Molecular Mechanics and Molecular Dynamics Simulations. J. Am. Chem. Soc. 1992, 114 (25), 10024-10035.

3. Horcas, I.; Fernández, R.; Gomez-Rodriguez, J.; Colchero, J.; Gómez-Herrero, J.; Baro, A., WSXM: a Software for Scanning Probe Microscopy and a Tool for Nanotechnology. Rev. Sci. Instrum. 2007, 78 (1), 013705.

4. Guinier, A.; Fournet, G., Small-Angle Scattering of X-Rays. John Wiley \& Sons, Inc.: New York, 1955.

5. Li, Y.; Wu, H.; Teng, P.; Bai, G.; Lin, X.; Zuo, X.; Cao, C.; Cai, J., Helical Antimicrobial Sulfono- $\gamma-$ AApeptides. J. Med. Chem. 2015, 58 (11), 4802-4811.

6. Wang, L.; Liu, R.; Gu, J.; Song, B.; Wang, H.; Jiang, X.; Zhang, K.; Han, X.; Hao, X.-Q.; Bai, S.; Wang, M.; Li, X.; Xu, B.; Li, X., Self-Assembly of Supramolecular Fractals from Generation 1 to 5. J. Am. Chem. Soc. 2018, 140 (43), 14087-14096.

7. Zhang, Z.; Wang, H.; Wang, X.; Li, Y.; Song, B.; Bolarinwa, O.; Reese, R. A.; Zhang, T.; Wang, X.-Q.; Cai, J.; Xu, B.; Wang, M.; Liu, C.; Yang, H.-B.; Li, X., Supersnowflakes: Stepwise Self-Assembly and Dynamic Exchange of Rhombus Star-Shaped Supramolecules. J. Am. Chem. Soc. 2017, 139 (24), 8174-8185. 8. (a) Schulze, B. M.; Watkins, D. L.; Zhang, J.; Ghiviriga, I.; Castellano, R. K., Estimating the Shape And Size Of Supramolecular Assemblies by Variable Temperature Diffusion Ordered Spectroscopy. Org. Biomol. Chem. 2014, 12 (40), 7932-7936; (b) Giuseppone, N.; Schmitt, J.-L.; Allouche, L.; Lehn, J.-M., DOSY NMR Experiments as a Tool for the Analysis of Constitutional and Motional Dynamic Processes: 
Implementation for the Driven Evolution of Dynamic Combinatorial Libraries of Helical Strands. Angew. Chem. Int. Ed. 2008, 47 (12), 2235-2239. 